\title{
An Optimized and Efficient Routing Protocol Application for IoV
}

\author{
Kiran Afzal, ${ }^{1}$ Rehan Tariq $\mathbb{D}^{2},{ }^{2}$ Farhan Aadil, ${ }^{2}$ Zeshan Iqbal $\mathbb{D}^{2},{ }^{2}$ Nouman Ali $\left(\mathbb{D},{ }^{3}\right.$ \\ and Muhammad Sajid ${ }^{4}$ \\ ${ }^{1}$ Department of Computer Science, COMSATS University Islamabad, Attock Campus, Attock 43600, Pakistan \\ ${ }^{2}$ Department of Computer Science, University of Engineering and Technology Taxila, Taxila 47050, Pakistan \\ ${ }^{3}$ Department of Software Engineering, Mirpur University of Science and Technology (MUST), Mirpur 10250, Pakistan \\ ${ }^{4}$ Department of Electrical Engineering, Mirpur University of Science and Technology (MUST), Mirpur 10250, Pakistan
}

Correspondence should be addressed to Nouman Ali; nouman.ali@live.com

Received 19 March 2021; Revised 23 April 2021; Accepted 5 May 2021; Published 19 May 2021

Academic Editor: Teen-Hang Meen

Copyright (c) 2021 Kiran Afzal et al. This is an open access article distributed under the Creative Commons Attribution License, which permits unrestricted use, distribution, and reproduction in any medium, provided the original work is properly cited.

IoV is the latest application of VANET and is the alliance of Internet and IoT. With the rapid progress in technology, people are searching for a traffic environment where they would have maximum collaboration with their surroundings which comprise other vehicles. It has become a necessity to find such a traffic environment where we have less traffic congestion, minimum chances of a vehicular collision, minimum communication delay, fewer communication errors, and a greater message delivery ratio. For this purpose, a vehicular ad hoc network (VANET) was devised where vehicles were communicating with each other in an infrastructureless environment. In VANET, vehicles communicate in an ad hoc manner and communicate with each other to deliver messages, for infotainment purposes or for warning other vehicles about emergency scenarios. Unmanned aerial vehicle(UAV-) assisted VANET is one of the emerging fields nowadays. For VANET's routing efficiency, several routing protocols are being used like optimized link state routing (OLSR) protocol, ad hoc on-demand distance vector (AODV) routing protocol, and destination-sequenced distance vector (DSDV) protocol. To meet the need of the upcoming era of artificial intelligence, researchers are working to improve the route optimization problems in VANETs by employing UAVs. The proposed system is based on a model of VANET involving interaction with aerial nodes (UAVs) for efficient data delivery and better performance. Comparisons of traditional routing protocols with UAV-based protocols have been made in the scenario of vehicle-to-vehicle $(\mathrm{V} 2 \mathrm{~V})$ communication. Later on, communication of vehicles via aerial nodes has been studied for the same purpose. The results have been generated through various simulations. After performing extensive simulations by varying different parameters over grid sizes of $300 \times 1500 \mathrm{~m}$ to $300 \times 6000 \mathrm{~m}$, it is evident that although the traditional DSDV routing protocol performs $14 \%$ better than drone-assisted destination-sequenced distance vector (DA-DSDV) when we have number of sinks equal to 25 , the performance of drone-assisted optimized link state routing (DA-OLSR) protocol is $0.5 \%$ better than that of traditional OLSR, whereas drone-assisted ad hoc on-demand distance vector (DA-AODV) performs $22 \%$ better than traditional AODV. Moreover, if we increase the number of sinks up to 50, it can be clearly seen that the DA-AODV outperforms the rest of the routing protocols by up to $60 \%$ (either traditional routing protocol or drone-assisted routing protocol). In addition, for parameters like MAC/PHY overhead and packet delivery ratio, the performance of our proposed drone-assisted variants of protocols is also better than that of the traditional routing protocols. These results show that our proposed strategy performs better than the traditional VANET protocols and plays important role in minimizing the MAC/PHY and enhancing the average throughput along with average packet delivery ratio.

\section{Introduction}

IoV is the new form of VANET and is the alliance of Internet and IoT. VANET is a type of wireless network where vehicles interact with each other as well as with roadside units within a short distance [1]. For the avoidance of human loss and to minimize the time being waste, everyone wants a traffic environment that has fewer chances of accidents and collision, with a more reliable path that could help us to avoid any delay caused by the traffic congestion [2]. Moreover, a 
reliable and quick communication is also an ample demand in disaster or emergency scenarios [3]. Some of the critical issues that make such communication difficult are physical hindrance including on road obstacles, mobility issues, limited range of vehicles, and cost of infrastructure installation. Such factors not only result in unreliable communication, but also in some cases totally make it impossible for vehicles to communicate efficiently. For an efficient communication in a vehicular environment, we must keep in mind some of the factors like the following:

(i) No. of possible paths

(ii) Turns

(iii) Intersections

(iv) Traffic congestion

(v) The nearest route to the destination

Several routing techniques like ant colony optimization have been used for this purpose [4]. The selected optimized path, that is, the shortest one, is tested again and again using route planning software available. Optimization can be gained based on heuristics which are gained through experience and provide us with efficient solutions. VANET comes under the category of mobile ad hoc network (MANET) that is a subclass of wireless ad hoc networks. Moving vehicles in VANET operate in two basic architecture modes: V2V (vehicle-to-vehicle) communication and V2I (vehicle to infrastructure) communication [5]. In the former architecture, the vehicles communicate with each other, to exchange information, through Dedicated Short Range Communication (DSRC) protocol, while in the latter architecture the communication between vehicles is via roadside units [6]. Vehicular ad hoc network has a highly dynamic topology with varying the speed of the vehicle, the number of vehicles, and the direction changed by the vehicles [2]. Due to such issues and those mentioned previously, a new class of ad hoc networks has been devised "Internet of Vehicles." It makes use of unmanned aerial vehicles which proved to be helpful in efficient communication between vehicles. In this paper, we have devised an optimized solution for enhancing the network efficiency in terms of better throughput, average packet delivery ratio, and less MAC/PHY overhead. Such proposed scheme will not only help in having better network experience in traffic, but also enhance the medicine and healthcare, agriculture, disaster, and emergency scenarios and provide environmental and surrounding information and a better solution for communication over a congested road. The topological constraint changes made differentiate our proposed scheme from those proposed earlier.

The remainder of the paper consists of the following sections: Section 2 involves introduction and brief explanation of the field of IoV. Section 3 discusses the routing problems and challenges, mobility models, application, and related work done by the researchers in the past. The proposed methodology is discussed in Section 4. The results are presented in Section 5. Section 6 throws light on the comparative analyses of the scenarios used in the proposed research. Lastly, the whole research is concluded under Section 7 along with intended future work.

\section{Internet of Vehicles (IoV)}

IoV is a special class that falls under the category of VANETs and IoT. This class constitutes the framework of vehicles that interact with each other for the sake of exchanging useful information about the traffic, roads, and environment around them. The interaction can be through the infrastructure using RSU (roadside units) which is vehicle to infrastructure communication, or the exchange is directly between the vehicles themselves adapting the vehicle-tovehicle communication mode. In IoV, vehicles communicate not only with other vehicles but also with the infrastructure, the handheld devices being carried by the pedestrians, the cloud servers, and the sensors deployed in the environment or within the vehicles themselves.

Due to traffic problems like traffic congestion, delays, and route optimization, there is a need to find some vehicle mobility pattern or routing protocols that can resolve these issues. Many routing protocols have been proposed, but not all of them can give our desired results, nor is each protocol best suited for vehicular ad hoc networks. One of the main problems that hinder vehicles from reaching their desired destination is the nonavailability of an optimized route. Due to frequently dynamic topology, there are frequent disconnections between vehicles. Moreover, the hindrance caused by tall buildings and physical objects makes it difficult for vehicles to receive data or to communicate with each other efficiently. One of the possible solutions can be the use of aerial nodes. Such nodes deployed at certain ranges might give us some better results, and performance might increase. To enhance the overall efficiency of a network, researchers work on some fundamental parameters like average throughput, packet delivery ratio, communication delay, MAC/PHY overhead, overall network congestion, and packet drop. By doing so, the coverage of vehicular nodes can be enhanced. Such nodes can be deployed at certain ranges for performance gains. In our intended work, we have proposed a model in which first we have analyzed the efficiency of different routing protocols where the vehicular nodes communicate with each other, scenario (a). Later, the results are generated in scenario (b) where the vehicles communicate with each other indirectly via some aerial nodes deployed at some distance. The results of both scenarios are compared and evaluated to determine which scenario gives us better results. Figure 1 shows a brief description of our desired scenarios. The focus of our research is mainly on the following:

(i) Utilizing the UAVs to evaluate the performance of traditional VANET routing protocols.

(ii) Evaluating average packet delivery ratio in traditional VANET by incorporating UAVs.

(iii) Minimizing the MAC/PHY overhead.

(iv) Maximizing the average throughput. 


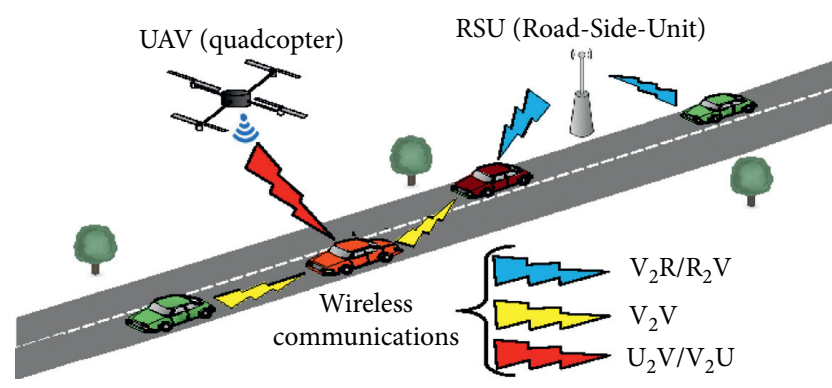

Figure 1: Scenarios a and b.

The end results of simulations show that our proposed strategy have better performance in terms of average packet delivery ratio, MAC/PHY overhead, and average throughput for larger grid size involving a greater number of vehicular as well as sink nodes.

\section{Related Works}

For years, the researchers from the academic field as well as from industry are investigating certain possible ways to solve the problems which are being faced in VANETs. Surveys have been done and algorithms have been proposed to provide optimized solutions for data delivery, better throughput, and optimized path. This section will focus on the relevant literature based on problems and challenges faced in vehicular ad hoc networks, Internet of Vehicles, mobility models used, and applications of such networks. We will also discuss some strategies or methodologies proposed by various researchers to minimize the issues being faced in this field.

3.1. Routing Problems and Challenge. Despite always ongoing research, certain challenges are still being faced in VANET including security, safety, and low latency. In the following subsections, we will discuss some of the problems which affect the network performance of VANET as well as IoV.

3.1.1. Route Optimization. One of the main issues in vehicular ad hoc networks is route optimization. Route optimization is basically about determining the efficient route in terms of less cost and shortest path with less amount of delay. For route optimization, we should keep in mind some of the factors like the no. of possible paths, turns, intersections, traffic congestion, and nearest route to the destination. Due to the highly dynamic topology and unpredictable movement of vehicles, it has become necessary to propose such a routing strategy that can mitigate such issues for better dissemination of information within interacting vehicles and the surrounding environment. As we have a broader range of possibilities, we mostly use algorithms which reduce the possibilities and help us to choose an optimized route (in terms of cost and energy consumption).
3.1.2. Security Issues. As the data being transferred in any network are crucial to its users, to have a secure network becomes a necessity. In VANETs, when two or more vehicles are communicating and sharing their information, they may face the interference of any hacker or intruder which could breach the network security by hacking the information flowing in the network (between the vehicular nodes) [7]. It has become a common cyber issue in the modern world as hackers and crackers are utilizing advanced and modern technologies to steal the information flowing in a vehicular environment. These cyber attacks can be active as well as passive nature including DOS attacks, revealing the identity, tracking and tracing of the vehicle's movement in the network, and black hole attacks.

3.1.3. Network Scalability. The scalability of VANET raises many critical issues. In the modern era, most people prefer to use their private transport, which results in an extensive and complicated network due to the increased number of vehicular nodes in VANET topology. Such an increase makes it difficult for the routing protocol to fully cover all the moving vehicles [8]. This affects the overall performance of the network where some of the areas of the network are under the control of the routing protocol and work efficiently, whereas, due to intensive network scalability, some of the vehicles are deprived of the efficient routing protocol.

3.1.4. Fluctuating Node Density. The vehicular node density in VANET is always unpredictable as the vehicles are always entering or leaving the network. Moreover, one cannot exactly predict in which part of the day the node density will be higher or will be less dense. Some of the routes in VANET are heavily dense due to route characteristics like better road conditions and shortest path to a certain destination. Such a condition can congest the traffic network, increasing network complexity, while the other routes may be sparsely dense which results in uneven node distribution in the network [9].

3.1.5. High Mobility and Dynamic Topology. Due to always moving vehicles that are entering the network as well as exiting it, the topology of vehicular ad hoc networks is always changing. Such type of mobility and topological changes make it difficult to have an optimized routing, and routing protocols which are well efficient in handling such types of routing problems in VANET are required.

3.2. Mobility Models. Depending upon the network requirements, a variety of mobility models have been proposed for the vehicular ad hoc network, each of which has its characteristics. In the following subsections, we will discuss some of the commonly used mobility models for VANETs. There are certain characteristics which are necessarily needed to build up an efficient mobility model. These characteristics may include the pattern in which mobility within the network is carried out, the average speed with which a vehicle can move in a network, and the mechanisms 
which can control the traffic. Depending upon such characteristics, a mobility model is selected and adapted for the intended network [10].

3.2.1. Random Waypoint Model (RWM). Random waypoint model is commonly used for ad hoc networks. Its main characteristics include simplicity and availability at a wide range. In RWM, the nodes can move freely without any limitation and restriction. Parameters like speed and direction of the nodes are chosen randomly. Along with its pros, there are two major issues of the random waypoint model: sudden stop and rapid change of directions [11].

3.2.2. Stop Sign Model (SSM). In the stop sign model, the moving vehicles make their movement relative to the traffic sign when they reach any type of intersection on the road. When a moving vehicular node reaches the intersection, that node must wait for a certain specified interval of time before heading towards its next destination. The vehicular node keeps distance from the node that is moving in front of it [7].

3.2.3. Probabilistic Traffic Sign Model (PTSM). The probabilistic traffic sign model uses traffic lights instead of utilizing the stop sign on the road when it reaches the junction. When a vehicular node approaches the junction, it has to wait for a randomly selected amount of time interval. In the same way, the vehicle that reaches this node has to wait again for a second, which increases the delay. The described model is useful as it decreases the excessive wait [10].

3.2.4. Manhattan. Manhattan mobility model works on the maps and is mostly preferred for urban environments. The maps used in the Manhattan mobility model use roads with different lanes, and each of these lanes has further two directions. Therefore, overall a node can move in four possible directions, that is, from north to south, from south to north, from east to west, and from west to east. Even a vehicular node can change its direction from left to right or from right side to left one when it will reach any kind of intersection. There is a $50 \%$ possibility that a moving vehicle will stay on the road, while the possibility of taking a turn is even half of it [12].

3.2.5. Freeway Mobility Model. The freeway mobility model operates on the behavior in which the vehicular nodes are moving on different types of freeways. As we know, there are several lanes on any freeway, and even those lanes have two types of directions separately for incoming and outgoing vehicles. In this model, each of the vehicular nodes is restricted to its specified lane. The speed with which nodes are moving is dependent on the speed of the previous node for a short time [13].

3.3. Applications. Vehicular ad hoc networks have a wide range of applications in different fields. With the advancement in modern technology, researchers have been adopting different methods to increase the utilization and applications of VANET. Such a network can be used for gaining information, for emergency scenarios, for entertainment, for safety, and for better utilization of roads in an efficient manner. Some of such characteristics have been listed below whereas more is yet to come.

3.3.1. Safety Purposes. As with the increasing number of vehicles on the road, there is an increasing risk of road accidents and vehicle collisions. Researchers have been working to deduce improved technologies for better traffic conditions. As in VANET the vehicles are communicating with each other, in case of any accidents the vehicles can generate warning or alerts so that the upcoming vehicles can be alerted. The drivers of vehicular nodes can easily be informed in advance about mishaps taken place on road.

3.3.2. Infotainment. Sometimes driving a car can be so boring, especially if you are moving on the same road on daily basis. Moreover, it could be difficult to travel around in an area if you do not have any information about that area. In such a case you will need to know about your location and nearest places or where your specified destination is. All problems like these can now be addressed by the vehicular ad hoc environment where the vehicular nodes are always in interaction with each other. Moreover, the interactive billboards and hoardings, downloads, notifications for the points, or things you are interested in can be appealing in VANET scenarios [14].

3.3.3. Emergency Scenarios. VANET is also well suited for disaster scenarios, as in such emergency scenarios, where any calamity has taken place like an earthquake or flooding, the infrastructure of the network deployed in that area partially or completely becomes inactive. Therefore, in such cases, the VANETs are helpful for communication with one and other as well as calling for help and services. Moreover, if there is something that could be dangerous for the upcoming vehicles like any wild animal that is present on the road and could be harmful, the drivers of those vehicles can be warned and stopped by sending them a warning or alert messages in a vehicular ad hoc network [14].

3.3.4. Management of Congested Traffic. For a smooth and safe traffic environment, the management of traffic is an important parameter. Consequently, to avoid congestion because of high node density, certain methods have been suggested for vehicular ad hoc networks like developing the application that can keep track of location information of the vehicles. This information is then shared with the drivers of the vehicles if they have installed the application [15]. Based on this received information, the driver can leave the congested road or may turn to any other route. 
3.3.5. Environmental Information. Like certain other applications, VANET provides the facility of dissemination of real-time data which may include alternative paths and weather conditions. In weather information, the driver can be provided with the information on the weather forecast and the possible adverse effects of the weather which may help to reduce the delays occurring due to adverse effects of weather like fog and rain [16].

3.4. Related Work. Several routing techniques like ant colony optimization have been used for this purpose [4]. The selected optimized path is tested again and again using route planning software available. Optimization can be gained based on heuristics which are gained through experience and provide us with efficient solutions.

Chen and coauthors proposed an efficient protocol that is designated to disseminate the data packet in the scenarios of urban areas while keeping various parameters in consideration like road traffic, topology, and information related to the specified geographical areas [17]. The protocol utilized the artificial spider web technology to discover the route between the source node and the destination, and it performs better in terms of end-to-end delay and packet delivery ratio. Nazib and Moh Reviewed various routing protocols that are most commonly used in vehicular environment with the assistance of aerial nodes [18]. The review has been done based upon the working mechanism and the principles adopted to design these protocols. The optimization and effectiveness of the protocols mentioned in this survey have also been discussed in detail.

Oubbati et al. proposed a reactive routing scheme which also involves the prediction method to select an efficient path to the desired or destination nodes. They have suggested the use of unmanned aerial vehicles for enhancing the effectiveness of the proposed scheme [19].

The usage of drone serving as relay node has been adopted in [20] by Lin et al. The aerial nodes have been distributed after predicting the number of vehicles participating in the ongoing traffic. This strategy considers several aspects of on-road traffic like non-line-of-sight and load on the network. Moreover, a new algorithm named as multimodal nomad algorithm is also proposed as an efficient solution to the problems in the vehicular environment involving aerial. The proposed model has slight loss in end-toend delay. Integrating the suggested model with other networks, such as the software-defined network, can enhance management and network control.

Kumar et al. suggested a heuristic algorithm for providing QoS in smart transportation system [21]. Although the proposed method enhances the network performance, it cannot be applied for a larger smart network. Lu et al. introduced an enhanced scheme for the city scenarios based upon geographical routing. The IGR scheme presented by the researchers works on two modes involving the greedy approach to forwarding data packets [22].

Bhatt et al. [23] suggested a model that uses the Bat algorithm to communicate with the destination by performing three stages using an optimized path. The first step of the proposed model is to predict where the destination is. In the second step, unnecessary or useless nodes are discarded, and a region is formed. In the last step, an optimized path among the multiple paths is selected. ACO was proposed by the Mexican researchers Dorigo et al. [24].

The central theme of ACO was taken from the social behavior of ants. Each ant in ACO represents one solution, and a group of multiple solutions or ants form the swarm. ACO encodes the real-world problem into a graph. Vertices of the graph correspond to a component of a candidate solution, and ants create a trail by traversing an edge. While traversing, ants diffuse some chemical substance, pheromone. The quantity of pheromone on the edge of the graph determines its quality. Ants add the component to its candidate solution by evaluating each edge of the graph. If the quality of the edge is better than others, ant traverses the edge and adds that vertex to the candidate solution. After some repetition of this procedure, the algorithm converges towards some candidate solution. Farhanchi et al. also proposed a model to figure out the shortest and optimized path [25]. Prakash used variant of two protocols [26]: the first protocol that has been used is P-OLSR for avoiding congestion, and the second is E-OLSR for balancing load and optimizing path.

In [27], Bao et al. arranged the nodes in clusters and determined an optimized path. A hybrid routing protocol that is road/path-aware and is assisted by the infrastructure has been discussed in [28]. It provides some key aspects like duration of the path, velocity of the moving vehicle, and transmission range. The model performs better in terms of packet delivery ratio and reduces delay with the help of predicting the duration of the path [29].

Jindal and Bedi combine the benefits of MACO and PSO algorithms to reduce travel time in VANET. West and Bowman uses ant colony optimization algorithm (ACO) to select the optimum path with better network connectivity in [3]. Zhang et al. applied the Q-learning algorithm to the parameter of link reliability, and its performance was analyzed. Based on these evaluations, a new strategy was proposed which performs better in terms of packet delivery ratio, transmission time, and frequent change of topology in VANET [30].

Tian et al. proposed a new model based on bioinspiration and is a unicast-routing protocol. It guarantees the efficiency of message delivery and the robustness of the overall system compared to prior conventional routing protocols [31]. Elhoseny and Shankar presented a model in which they utilized the K-Medoid Clustering for arranging the vehicles in the form of clusters. The nodes which have efficient energy are distinguished by utilizing the metaheuristic algorithm. Afterward, these nodes are used for communication [32].

Nayyar analyzed different protocols like AODV, OLSR, DSDV, DSR, AOMDV, and HWMP to evaluate their performance in the FANETs scenarios to use them in real operations [33]. Leonov tried to examine various approaches that are based on the bee colony algorithm. Results were analyzed and a new strategy, BeeAdHoc, that is comparatively better than traditional VANET protocols (AODV, DSDV, and DSR) was proposed [34]. Majumdar and 
coauthors tried to overcome the problem of high latency and unsuccessful delivery of data to the destination [35]. For this purpose, the advantages of the ant colony optimization technique were utilized. The use of artificial ants and artificial neighbors was considered to enhance the discovery of new paths.

Considering various parameters, the pros and cons of different protocols which are being utilized in FANETs have been discussed by Oubbati et al. [36]. Furthermore, future challenges have been discussed as well, which could be considered for research study and work. The focus of this paper is mainly on position-based routing protocols for FANETs. Saritha et al. proposed a new algorithm based on particle swarm optimization, leapfrog, and learning automata in [37].

The proposed strategy is supposed to find multiple paths for the data delivery considering the link stability. Leapfrog helps in determining the link failure in advance for avoiding any data loss. The results thus gathered show that the proposed algorithm performs better in terms of a better packet delivery ratio.

In [38], Bravo-Torres et al. proposed a virtual node layer which lies between the link layer and Internet layer which can enhance the work of AODV. The newly adapted AODV is termed as VNAODV which can give better results in a vehicular ad hoc environment. Dixit et al. surveyed the VANET architecture in [39]. They provided the research challenges and details about different routing protocols being adopted. Application and algorithms for VANET scenarios have also been discussed.

Maistrenko et al. tried to compare AODV, DSDV, and DSR with AntHocNet routing protocol [40]. After performing simulations, it was concluded that AntHocNet performs better, as the other three experimented protocols have low performance with highly mobile nodes.

For maximizing the throughput, Zeng and others presented a novel technique of embedding the sink or relay nodes over the aerial nodes. Due to such projection, the relay nodes were able to fly with great speed. This technique of utilizing the mobile relay nodes has enhanced the throughput gained as compared to the traditional relaying where the nodes acting as relay are static in wireless communications [41].

A novel technique has been proposed by Mozaffari for collection of data from the IoV that has been deployed or are being used on the ground via unmanned aerial vehicles deployed or moving in all the three dimensions. The technique resulted in better transmission power and data collection as compared to the conventional stationary aerial nodes deployed at a height from the ground [42].

For better performance in intelligent transportation systems, Yasser and coauthors have proposed a new strategy that can help the people living in developing countries or areas where there is lack of roadside units. They have utilized the vehicle-to-vehicle communication as freestanding system for intelligent transportation system. Different proactive and reactive routing protocols have been tested without the usage of RSU. The real-world simulations were performed with the utilization of OPNET simulator, and finally the simulation results showed that utilizing such standalone system without roadside units has better performance for developing areas with utilization of AOD protocol [43].

\section{Proposed Methodology}

The proposed model's framework is shown in Figure 2. At the start, the network has been created by deploying only the vehicles with a certain transmission range. Certain parameters were set as per our requirement. We implemented our proposed strategy after evaluation of traditional VANET routing protocols, i.e., OLSR, AODV, and DSDV. We called this phase scenario a and its steps are listed below:

(i) Routing protocols' selection

(ii) Direct vehicular communication without the assistance of aerial nodes

(iii) Results' generation

All the above-described steps followed the routing procedure in the traditional VANET. Here, scenario a of our proposed strategy ends, and we move to scenario $b$ which is communication via aerial nodes. The steps involved in the routing of scenario $b$ are as follows:

(i) Changing altitude of sinks and deploying them as aerial nodes

(ii) Communication between vehicles via these deployed aerial nodes

(iii) Generation of results

A detailed discussion of both scenarios is as follows.

\subsection{Routing by Using Traditional Vehicular Ad Hoc Network (Scenario A)}

4.1.1. Selection of Routing Protocol. In our simulation, we considered OLSR, AODV, and DSDV protocol to check their efficiency in our first scenario. Each protocol depicted different results in terms of average throughput, average packet drop ratio, and MAC/PHY overhead.

4.1.2. Direct Vehicular Communication. In the first phase of our simulation, the deployed vehicles communicate with each other without the assistance of any aerial node. The vehicles have certain transmission ranges, some speed, and basic service message route from node to node to be delivered to our desired destination. These messages are initiated by a certain source.

4.1.3. Generation of the Result. Once the protocols have been selected, the simulations were performed, and the results were generated in the form of a graph for later comparison.

\subsection{Routing via Aerial Nodes (Scenario B)}

4.2.1. Changing Altitude of Sink Nodes. After all the procedures described above, we proceed ahead towards scenario b where we have changed the height of sink nodes to deploy 


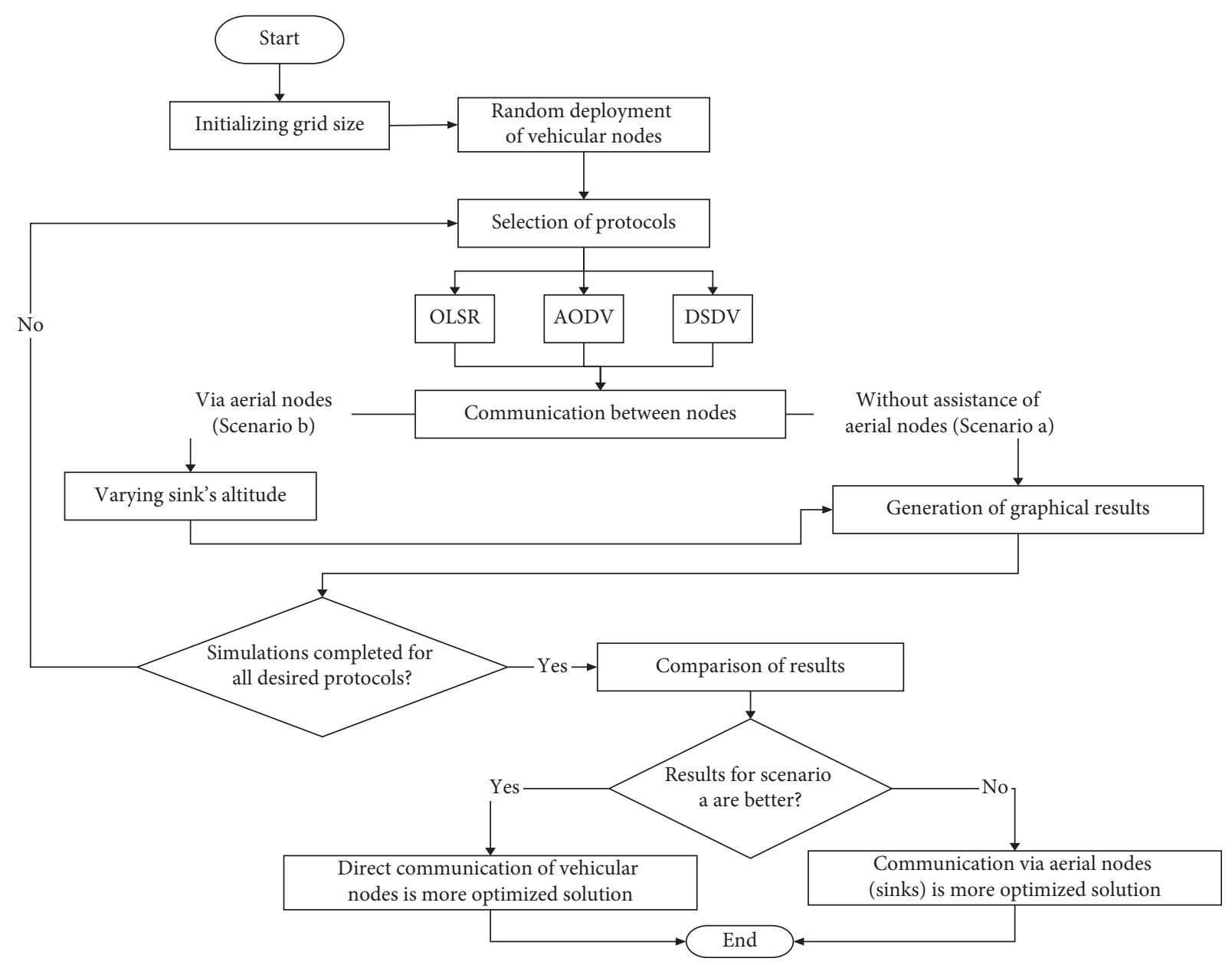

FIGURE 2: Framework of the proposed methodology.

them as aerial vehicles at some range and some altitude. The number of aerial nodes can be different for different scenarios. These aerial nodes have their specifications and parameters.

4.2.2. Communication via Aerial Nodes. Once the sink nodes have been deployed as aerial nodes, vehicles start communicating with each other indirectly via aerial vehicles. Such indirect communication can help us in an environment where vehicles are at such a distance from each other that they are unable to communicate directly with each other. Hence, such distanced vehicles can deliver their messages indirectly via these elevated sink nodes. These aerial vehicles have certain number and certain specifications like speed and altitude power consumption.

4.2.3. Generation of Results in Graphical Form. Once the simulations have been performed keeping scenario $b$ under consideration, we have generated graphs. These graphs help us in comparing our two scenarios.

4.3. Simulation Scenario. All the simulations have been carried out in synthetic highway scenarios. For the performance evaluation of our proposed strategy, simulations have been carried out in the NS3 simulator. For the analysis of the performance of OLSR, AODV, and DSDV protocols, in both scenarios, i.e., traditional vehicular ad hoc network and our proposed scenario of the Internet of Vehicles, the density of vehicles has been varied from low to high. It is done so that we can track the performance in an environment where we have congested traffic and where there is less vehicular traffic. The routing protocols that have been considered are OLSR, AODV, and DSDV. The rest of the parameters and their specifications are described in Table 1. The step-wise explanation of Figure 2 is mentioned as Algorithm 1 .

4.4. Description of Key Parameters. The key parameters involved in the simulation are described in Table 2. These parameters helped us in evaluating and analyzing our proposed strategy to decide whether the traditional vehicular ad hoc network performs better or the assistance of aerial nodes would be beneficial.

4.5. Evaluation Metrics. The evaluation of our proposed strategy has been done by keeping in mind the following metrics. 
TABLE 1: Simulation setup.

\begin{tabular}{lc}
\hline Parameters & Specification \\
\hline Operating system & Ubuntu-18.04.3 \\
MATLAB & R2015a \\
Simulator & NS3-3.30.1 \\
Scenario & VANET $(802.11 \mathrm{p})$ \\
Mobility model & Random way point \\
Speed of vehicles & $20 \mathrm{~m} / \mathrm{s}$ \\
Pause time & $300.01 \mathrm{~s}$ \\
Grid size & $300 \times 1500 \mathrm{~m}, 300 \times 4500 \mathrm{~m}, 300 \times 6000 \mathrm{~m}$ \\
Number of vehicular nodes & $100,200,300,400,500$ \\
WiFi & $802.11 \mathrm{p}$ \\
Control channel & $10 \mathrm{MHz}$ \\
Number of sink nodes & 25,50 \\
Loss model & Two-ray ground \\
Transmission power & $20 \mathrm{dBm}$ \\
Transmission range & $145 \mathrm{~m}$ \\
Total simulation time & $300.01 \mathrm{~s}$ \\
Antenna height along $z$-axis & $1.5 \mathrm{~m}$ (in scenario a), $50 \mathrm{~m}$ (in scenario b) \\
\hline
\end{tabular}

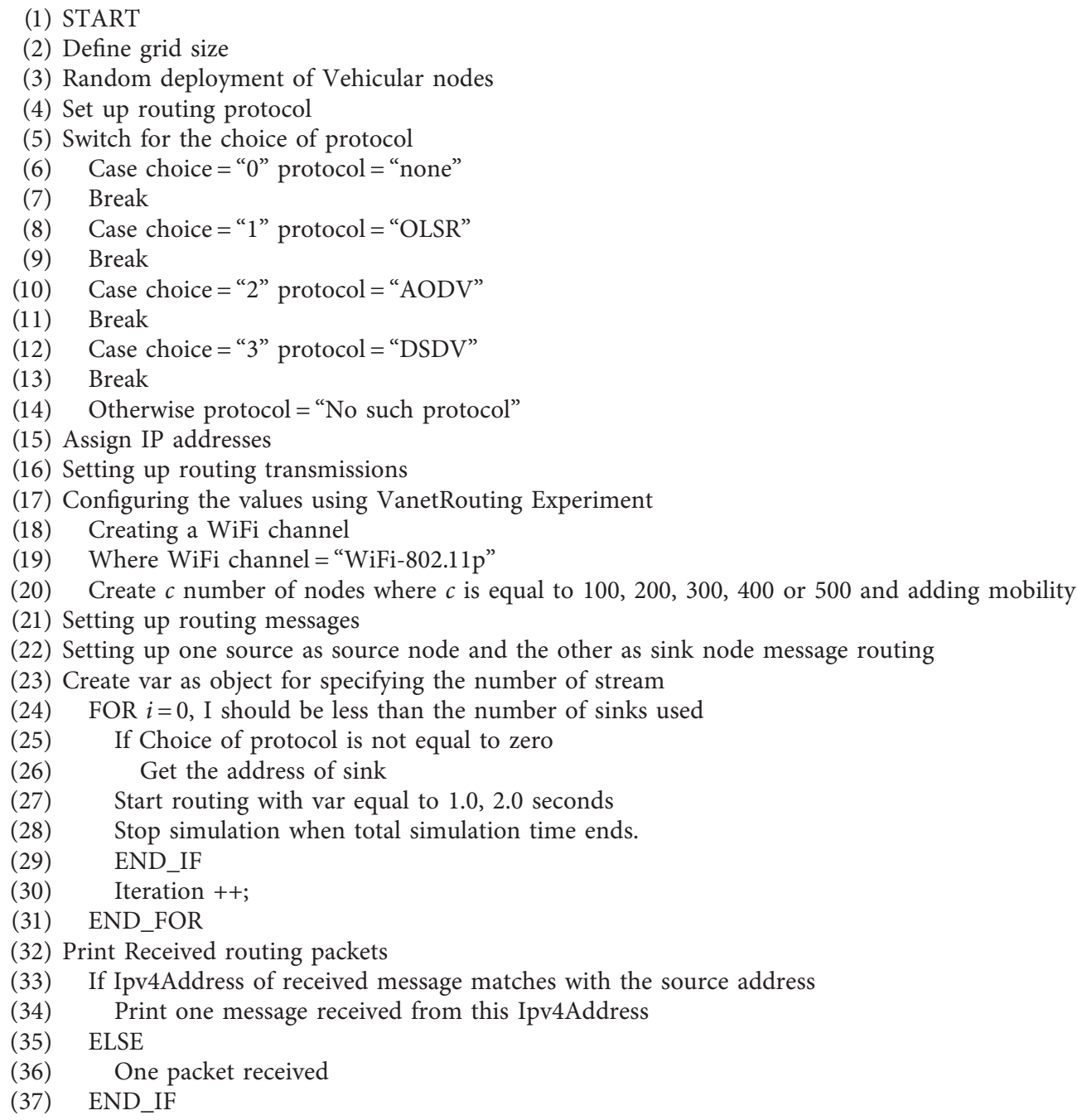


(38) Set receive call back to acknowledge packet received

(39) Logging

(40) VanetRoutingExperiment experiment ();

(41) WiFiApp ()

(42) SetDefaultAttributeValues (); set default values to all attributes

(43) ConfigureNodes (); configure all the nodes

(44) ConfigureChannels (); for configuration of channels

(45) ConfigureMobility (); configure the mobility

(46) ConfigureApplications (); for configuration of applications

(47) RunSimulations (); start and end simulations from zero seconds to the total simulation time

(48) ProcesssOutputs (); process the results obtained as output

(49) CourseChange (); set up sinks' velocity and position

(50) main ();

(51) VanetRoutingExperiment experiment ();

(52) END

Algorithm 1: UAV-assisted VANET routing protocol.

TABLE 2: Key parameters for simulation.

$\begin{array}{ll}\text { Parameters } & \text { Description }\end{array}$

Transmission range

Rate of transfer

Packet received

Packet size

Pause time

Simulation time

Received rate

Packet loss

No. of sinks

Grid size

Node speed

Node direction

Basic safety messages (BSM)
It defines the vehicle's range in which it would be able to communicate with other vehicles in the network. Varying the transmission range may impact the overall performance of the network.

It specifies how much data can be transferred in the given time.

This parameter describes the number of successful packets received by the destination. It greatly impacts the performance of any network.

Packet size may vary from network to network, but we will have a fixed size of packets that can be routed in the network.

It is the controlling parameter specifying how much time a sink node will stay in a specified grid.

The total time taken for one whole simulation is considered in this parameter.

This parameter tells us the amount of data received in bytes/kilobytes.

The number of packets that were not received by the destination due to communication error is calculated under the packet loss parameter.

Sinks are the nodes that help us to gather and preprocess the data collected from the surroundings via sensor node. The number of deployed sink nodes for the desired scenario is specified under this parameter.

The overall size of the grid in which the experimentation is done.

This is the movement speed of the node.

This is the direction of the vehicle on the road on which it is moving, it may include intersections and left, right, or straight lane.

These messages do not help in routing, but rather they provide other useful information, yet they consume bandwidth of the network.
4.5.1. Average Packet Delivery Ratio. The average packet delivery ratio in any scenario tells us about the ratio of the number of packets received by the destination to the total number of packets sent by the source. It is an important parameter as it helps us to evaluate the performance of any network. The higher the average packet delivery ratio is, the higher the reliability of that network will be.

$$
\text { average PDR }=\frac{\sum \text { no. of the packets received }}{\sum \text { no. of the packets sent }} .
$$

4.5.2. Average Throughput. Average throughput specifies, at any time, the amount of data sent from the source to the desired destination successfully. If we have a higher value of throughput, then the performance of our network will be enhanced. It can be calculated as

$$
\text { throughput }=\frac{\left.\left(\sum \text { (no. of successful packets }\right) *(\text { average packet size })\right)}{\text { transmission time }} .
$$

4.5.3. MAC/PHY Overhead. In vehicular ad hoc network, we use BSM that help us to share information in the network, whereas the information related to the updates of routing is disseminated by the routing packets. However, the routing packets do not provide any useful information related to the application. BSM as well as routing packets consume the bandwidth of the network, which affects the overall performance of the network. Hence, we call these routing packets causing an overhead on the network bandwidth as MAC/PHY overhead, and for their calculation, we need to know the total number of physical bytes and we should have 
information about the total application byte. The MAC/PHY overhead in scenario a and in scenario b can be calculated as

$$
\text { MAC/PHY Overhead }=\frac{\text { (totalPhyBytes }- \text { totalAppBytes })}{\text { (totalPhyBytes) }} \text {. }
$$

\section{Simulated Results}

\subsection{For Traditional Vehicular Ad Hoc Network}

5.1.1. MAC/PHY Overhead with 25 Sink Nodes. For all the graphs presented in Figure 3, we have kept the number of sink nodes initially equal to 25 , whereas vehicular nodes increase from 100 to 500 .

In Figure 3(a), we have grid size $=300 \times 1500 \mathrm{~m}$; MAC/PHY overhead ascends for OLSR as the number of nodes ascends/increases. In the case of AODV, MAC/PHY overhead descends when the number of nodes ascends from 100 to 200. It becomes constant when nodes increase from 200 to 300. Again, it ascends when the number of nodes ascends from 300 to 400 , and after that it becomes constant. In DSDV, when the number of nodes increases, MAC/PHY overhead firstly increases and then becomes constant. Again, it increases and after that shows constant behavior.

It is clear from Figure 3(b), where we have a grid size of $300 \times 3000 \mathrm{~m}$, that MAC/PHY overhead increases for OLSR with the increasing number of nodes. In the case of DSDV, MAC/PHY overhead ascends when the number of nodes ascends. In AODV, MAC/PHY overhead ascends when the number of nodes ascends from 100 to 200, it becomes constant for 200 to 300 nodes, and it ascends onwards.

Figure 3(c) shows that MAC/PHY overhead ascends for all the protocols of OLSR, AODV, and DSDV when the number of nodes increases while grid size is $300 \times 4500 \mathrm{~m}$. The MAC/PHY overhead is the highest for AODV and is the lowest for OLSR, whereas for DSDV it lies in between them.

Figure $3(\mathrm{~d})$ presents a scenario where the grid size is increased to $300 \times 6000 \mathrm{~m}$. In the case of OSLR, MAC/PHY overhead ascends when the number of nodes is up to 300 and becomes constant when the number of nodes increases from 400 to 500. In DSDV, MAC/PHY overhead ascends with increasing the number of nodes; in the case of AODV, MAC/PHY overhead increases when the number of nodes increases from 100 to 200; then, it becomes constant when the number of nodes ascends from 200 to 300; and after that it ascends gradually.

5.1.2. MAC/PHY Overhead with 50 Sink Nodes. For all the graphs presented in Figure 4, we have kept the number of sink nodes equal to 50 whereas vehicular node increases from 100 to 500 .

It is clear from Figure 4(a) that, in case of OLSR, MAC/PHY overhead descends gradually with the ascending number of nodes keeping the grid size equal to $300 \times 1500 \mathrm{~m}$. While in the case of DSDV, MAC/PHY overhead ascends from 200 to 300 , and there is a sudden increase when the number of nodes ascends from 200 to 300. After that,
MAC/PHY overhead descends with an ascending number of nodes. In the case of AODV, MAC/PHY overhead ascends when the number of nodes ascends from 100 to 200, after that it descends with ascending number of nodes till 400, and from 400 nodes onwards MAC/PHY overhead also ascends.

Figure 4(b) indicates that MAC/PHY the overhead of AODV is greater than those of the other two protocols, while OLSR has the least MAC/PHY overhead. The DSDV, the same as in the previous cases, lies between AODV and OLSR routing protocol. Grid size, in this case, has been increased from $300 \times 1500 \mathrm{~m}$ to $300 \times 3000 \mathrm{~m}$.

Figure $4(\mathrm{c})$ shows the results with a grid size equal to $300 \times 4500 \mathrm{~m}$. The results demonstrate that MAC/PHY overhead ascends with the ascending number of nodes in both OLSR and DSDV, but its behavior is different in the case of AODV. In AODV, MAC/PHY overhead ascends when the number of nodes increases from 100 to 300 and, after that, it descends with the ascending number of nodes.

From Figure 4(d), in grid size $300 \times 6000 \mathrm{~m}$, the MAC/PHY overhead for OLSR ascends slowly, with the ascending number of nodes. In DSDV, MAC/PHY overhead increases with the increasing number of nodes. In AODV, MAC/PHY overhead ascends when the number of nodes ascends from 100 to 300 , then becomes constant when nodes increase from 300 to 400, and again increases.

5.1.3. Average Throughput with 25 Sink Nodes. For all the graphs in Figure 5, we keep a constant number of sink nodes, i.e., 25, and the vehicular nodes increase from 100 to 500 . Grid size is incremented by $1500 \mathrm{~m}$ each time along the $y$ axis.

Figure 5(a), where the grid size is $300 \times 1500 \mathrm{~m}$, represents the average throughput of three protocols (OLSR, AODV, and DSDV). Throughput for OLSR rapidly increases from 100 to 200 and then gradually decreases with nodes from 200 to 500. Protocol AODV shows average throughput on the first 100 nodes; then, it decreases rapidly; after 200 nodes, it shows average performance for 400 nodes; and then its performance is enhanced from 400 to 500 nodes. In the case of DSDV, throughput increases as the number of nodes ascends from 100 to 200 rapidly, and then it starts descending as the number of nodes reaches from 200 to 300. It increases as the number of nodes increases, and then again it starts descending.

In Figure 5(b), we considered the grid size equal to $300 \times 3000 \mathrm{~m}$. OLSR shows the minimum average throughput as compared to AODV and DSDV. It remains constant from 100 to 200 nodes and then shows a gradual decrease as the number of nodes ascends, and after that it remains constant from 300 to 400 nodes. It shows a decrease in average throughput with nodes from 400 to 500. AODV shows a rapid increase in average throughput as the nodes ascend from 100 to 200 , and then its throughput decreases from 200 to 300 . After that, it shows a gradual decrease as the number of nodes goes up. DSDV shows a good increase in throughput from 100 to 300 , but it decreases as the nodes ascend from 300 to 400 . Then again, it shows an increase in the average throughput from 400 to 500 . 

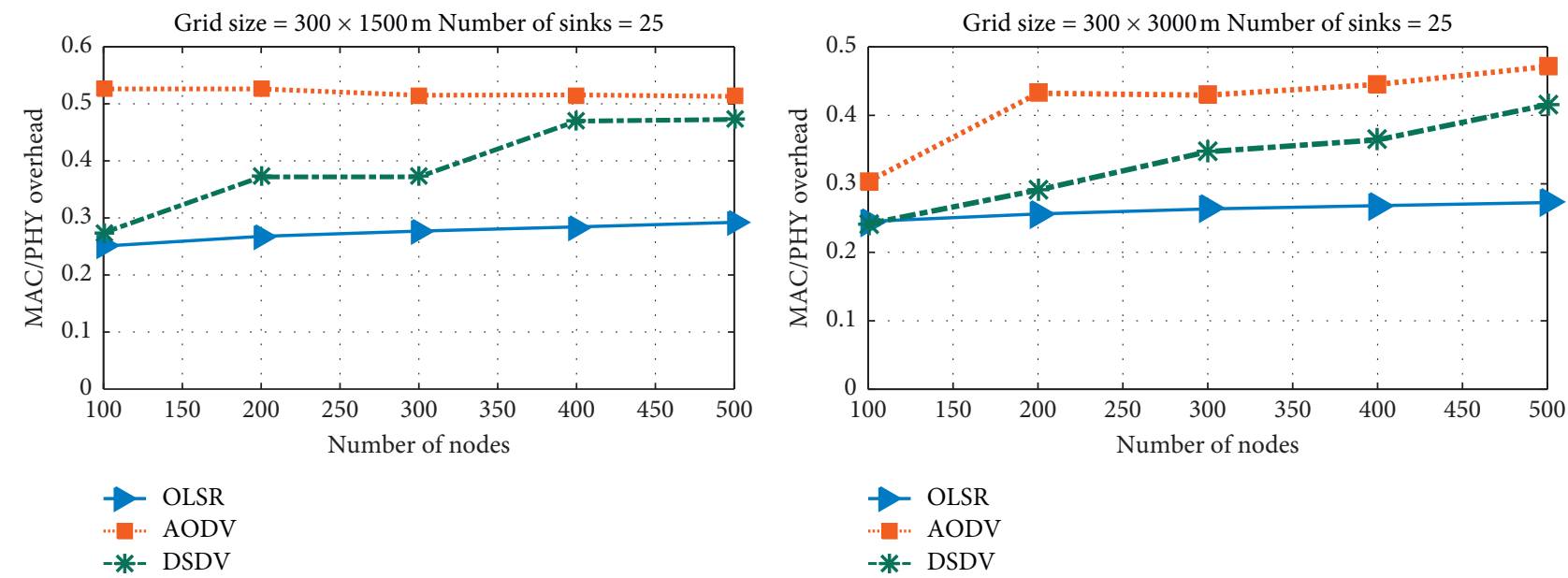

(a)

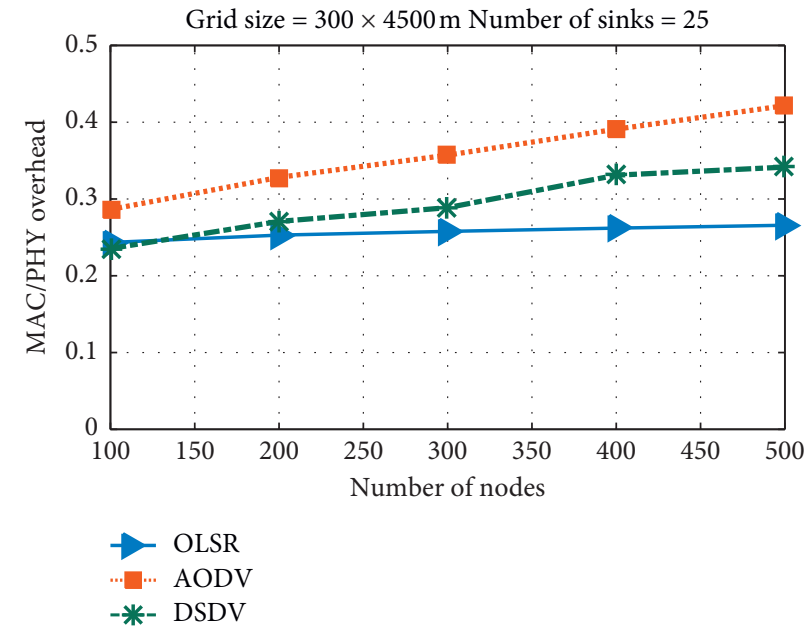

(c)

(b)

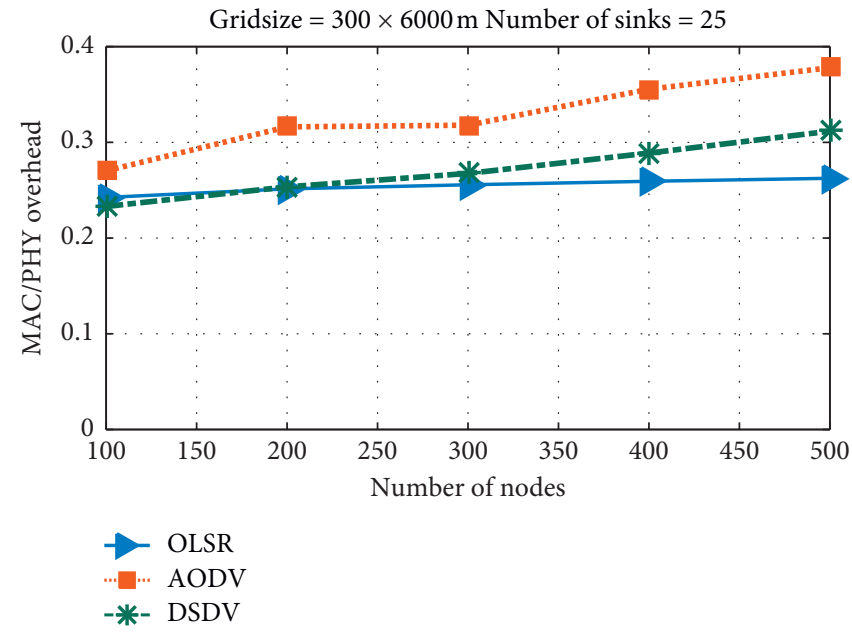

(d)

Figure 3: MAC/PHY overhead in traditional VANET with no. of sinks $=25$.

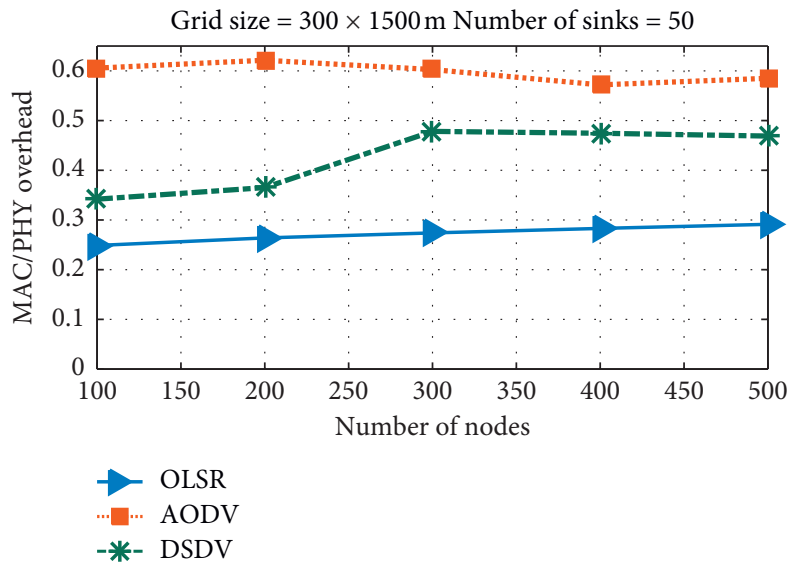

(a)

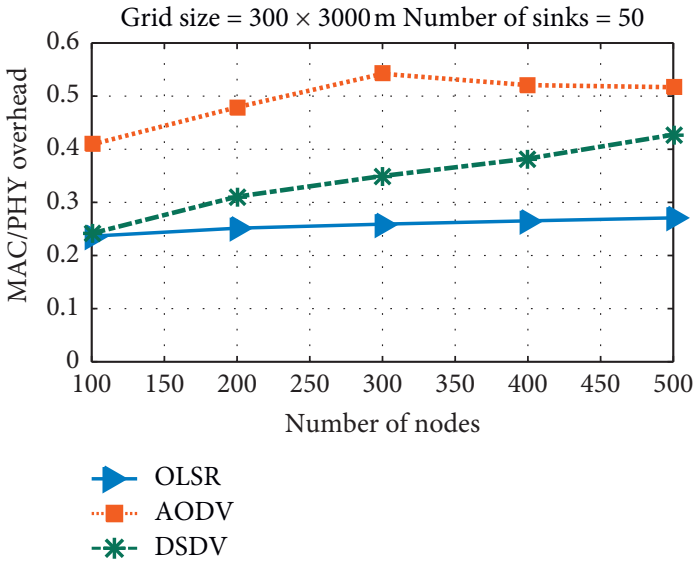

(b)

Figure 4: Continued. 


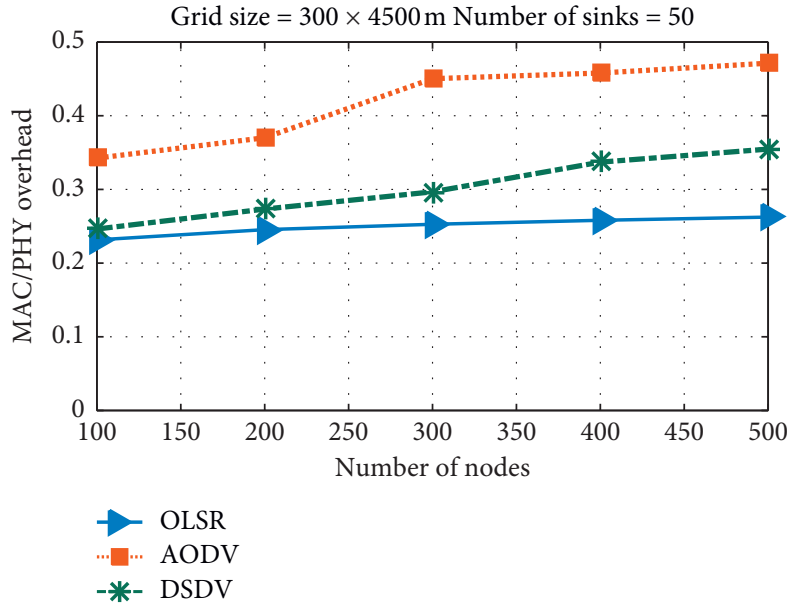

(c)

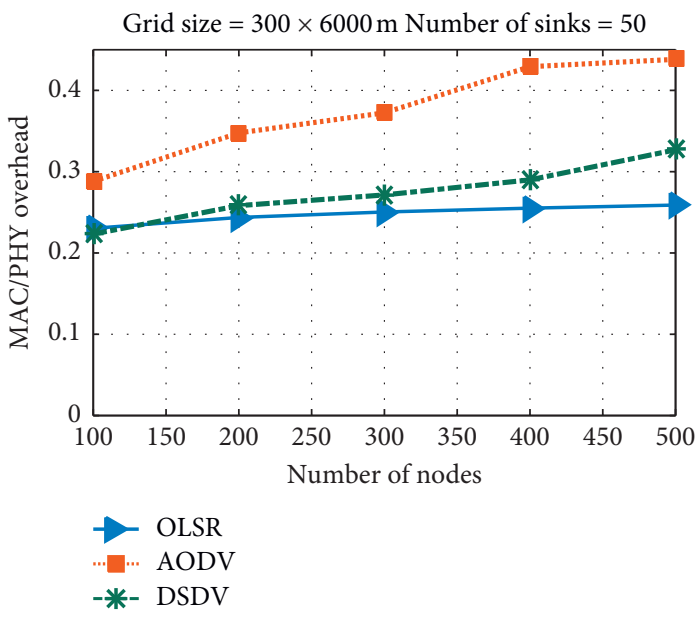

(d)

FIgURE 4: MAC/PHY overhead in traditional VANET with no. of sinks $=50$.

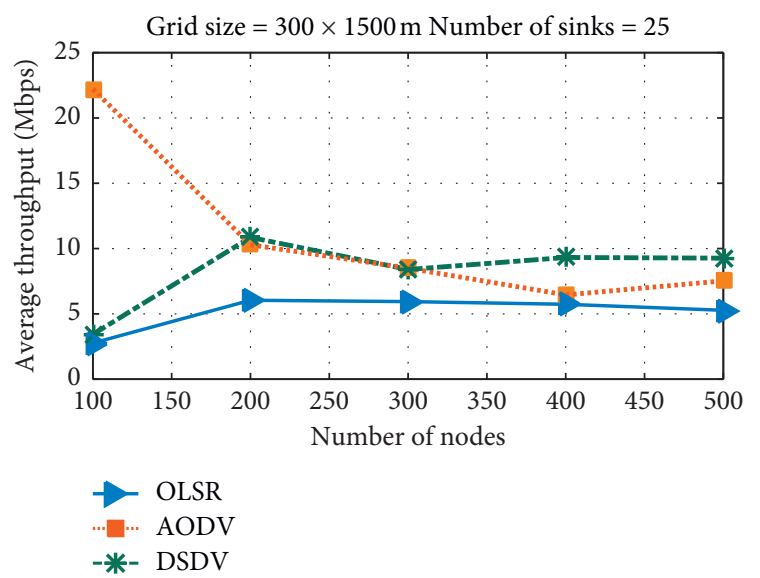

(a)

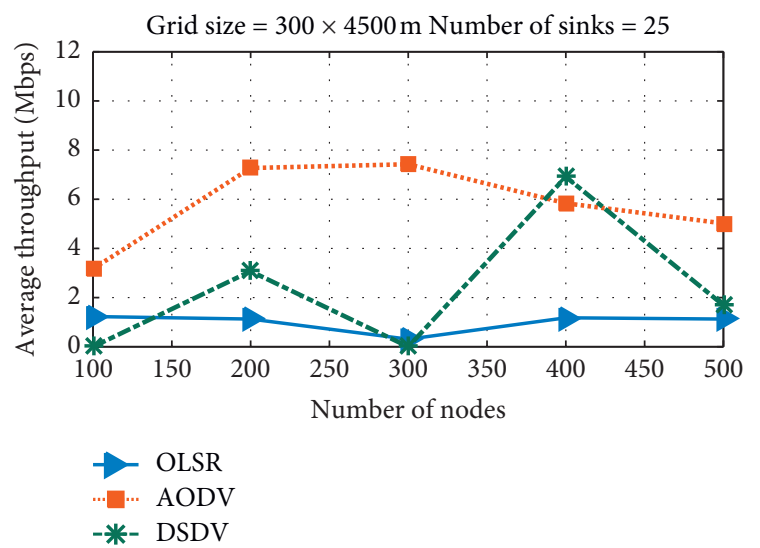

(c)

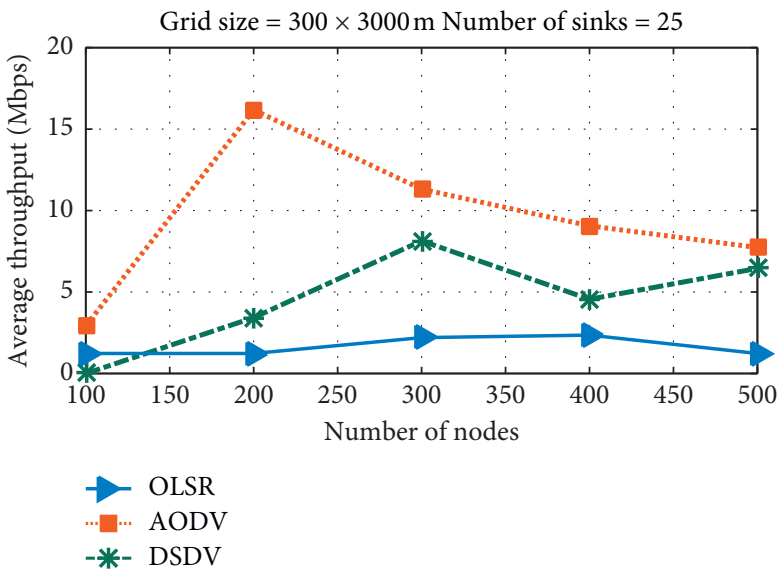

(b)

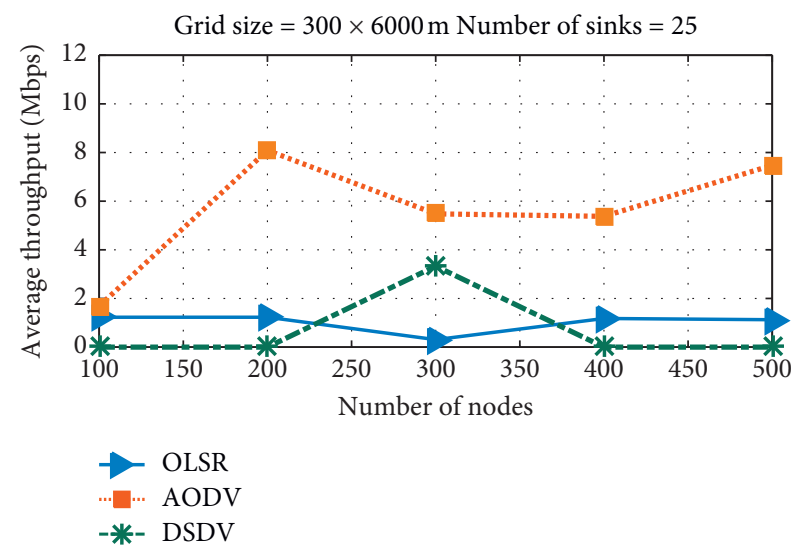

(d)

FIgURE 5: Average throughput in traditional VANET with no. of sinks $=25$.

From Figure 5(c), we can see that OLSR shows minimum throughput; it starts decreasing as the number of nodes ascends from 100 to 300 and then gradually increases as the number ascends. AODV shows high throughput overall as it increases from 100 to 300 but then starts decreasing from 300 to 500 . DSDV exhibits a rise in average throughput from 
100 to 200 nodes, then there is a fall in average throughput as nodes ascend, and its average throughput again starts rising with nodes from 300 to 400 . After 400 nodes, its average throughput decreases as the nodes ascend. The grid size in this scenario is $300 \times 4500 \mathrm{~m}$.

When we have a grid size of $300 \times 6000 \mathrm{~m}$, as shown in Figure 5(d), the DSDV protocol shows the lowest average throughput with nodes from 100 to 200 and from 400 to 500. On the other hand, the highest average throughput is depicted in the AODV protocol. OLSR operates in reverse to the DSDV protocol. Where there is a decrease in average throughput in the case of DSDV, the throughput for OLSR ascends. In the rest of the scenario, throughput for OLSR lies between the other two protocols.

5.1.4. Average Throughput with 50 Sink Nodes. For all graphs presented in Figure 6, the number of sinks is equal to 50, and the number of nodes increases from 100 to 500, whereas the initial grid size is $300 \times 1500 \mathrm{~m}$ which has an increase of $1500 \mathrm{~m}$ along the $y$-axis in the rest of the cases.

Figure 6(a) indicates that OLSR has the least average throughput when we have a grid size of $300 \times 1500 \mathrm{~m}$. AODV shows good performance on the first 100 nodes but then gradually decreases as the nodes ascend from 200 to 500. DSDV has a good average throughput as the nodes increase from 200 to 400. It has a comparatively less average throughput for the rest of the nodes.

OLSR almost remains constant with little rise and fall from 100 to 500 nodes within a grid of size $300 \times 3000 \mathrm{~m}$ as shown in Figure 6(b). AODV shows a decrease in average throughput with nodes from 100 to 200; then, there is an increase in average throughput as the number of nodes increases. It again slopes down with nodes from 300 to 400. Its average throughput remains constant with nodes from 400 to 500 . DSDV shows high average throughput as compared to AODV and OLSR with nodes from 100 to 500 .

Figure 6(c) represents simulations in grid size of $300 \times 4500 \mathrm{~m}$. OLSR gives low average throughput and almost remains constant with nodes from 100 to 500, with little rise and fall. AODV shows a high average throughput with nodes from 100 to 400, and then it gradually decreases as the nodes ascend. DSDV remains constant with nodes from 100 to 400, with a little increase, and decreases after that with nodes from 400 to 500. Anyhow, the best performance in this scenario is depicted in AODV, and OLSR protocol is least performing.

Figure 6(d) shows the average throughput of three protocols in the grid size of $300 \times 6000 \mathrm{~m}$. OLSR almost remains constant and gives the least throughput throughout the simulation with nodes from 100 to 500, with a little increase and decrease. DSDV shows average performance with the number of nodes from 100 to 300 and then increases as the number increases from 300 to 500. AODV performs best, and its throughput increases as the number of nodes increases from 100 to 300 and then gradually decreases with nodes from 300 to 500. Still, it behaves better than the other two protocols.
5.1.5. Average Packet Delivery Ratio with 25 Sink Nodes. For all the four grid sizes, i.e., $300 \times 1500 \mathrm{~m}$ to $300 \times 6000 \mathrm{~m}$, we have deployed 25 sink nodes for each case. The grid size increases with an equal interval of $1500 \mathrm{~m}$ along the $y$-axis each time. We have kept the number of vehicular nodes constant, that is, from 100 to 500 . Figures 7(a)-7(d) show the average packet delivery ratio of the three protocols, OLSR, AODV, and DSDV. In grid size of $300 \times 1500 \mathrm{~m}$, the performance of AODV is less than the other two protocols, whereas OLSR and DSDV show very close results. At 100 nodes, the three protocols give the highest average packet delivery ratio, but as we increase the number of vehicular nodes, there is a decrease in average packet delivery ratio in all the four grid sizes. However, it is obvious from Figures 7 (a)-7(d) that the three protocols are giving a better average packet delivery ratio as we increase the grid size each time. The performance of the three protocols is better at grid size $300 \times 6000 \mathrm{~m}$ than that at $300 \times 1500 \mathrm{~m}$. Accordingly, we can conclude that the average packet delivery ratio increases as we increase the grid size.

5.1.6. Average Packet Delivery Ratio with 50 Sink Nodes. For all the four grid sizes, i.e., $300 \times 1500 \mathrm{~m}$ to $300 \times 6000 \mathrm{~m}$, we have deployed 25 sink nodes for each case. The grid size increases with an equal interval of $1500 \mathrm{~m}$ along the $y$-axis each time. We have kept the number of vehicular nodes constant, that is, from 100 to 500. From Figure 8(a), we can see that, at grid size of $300 \times 1500 \mathrm{~m}$, the OLSR and DSDV have the highest performance with 100 vehicular nodes, but as we ascend towards vehicular nodes equal to 500, the average packet delivery ratio decreases. The performance of AODV at this point is less than that of the other two protocols. Later, when we increase the grid size up to $300 \times 3000 \mathrm{~m}, 300 \times 4500 \mathrm{~m}$, and $300 \times 6000 \mathrm{~m}$, respectively, the DSDV protocol behaves better than the other two, as could be seen from Figures 8(b)-8(d).

5.2. For Drone-Assisted Vehicular Ad Hoc Network. After completing the simulations for traditional VANET, we performed extensive simulations for scenario $b$ where we have made use of aerial vehicles. Results are computed against MAC/PHY overhead, average throughput, and average packet delivery ratio.

\subsubsection{MAC/PHY Overhead with 25 Sink Nodes.} Simulated results for MAC/PHY overhead in our scenario b with the assistance of aerial nodes are presented in Figures 9(a)-9(d). Here, the number of sink nodes is equal to 25 , and vehicular nodes are taken from 100 to 500. It is clear from Figure 9(a) that MAC/PHY overhead for DA-DSDV increases when the number of nodes increases from 100 to 300. It shows a decrease in performance with nodes from 300 to 40 and, later on, there is an enhancement in its performance once again. MAC/PHY overhead for DA-AODV ascends when the number of nodes ascends from 100 to 200, and it descends when the number of nodes ascends from 200 to 400 and becomes constant after that. 


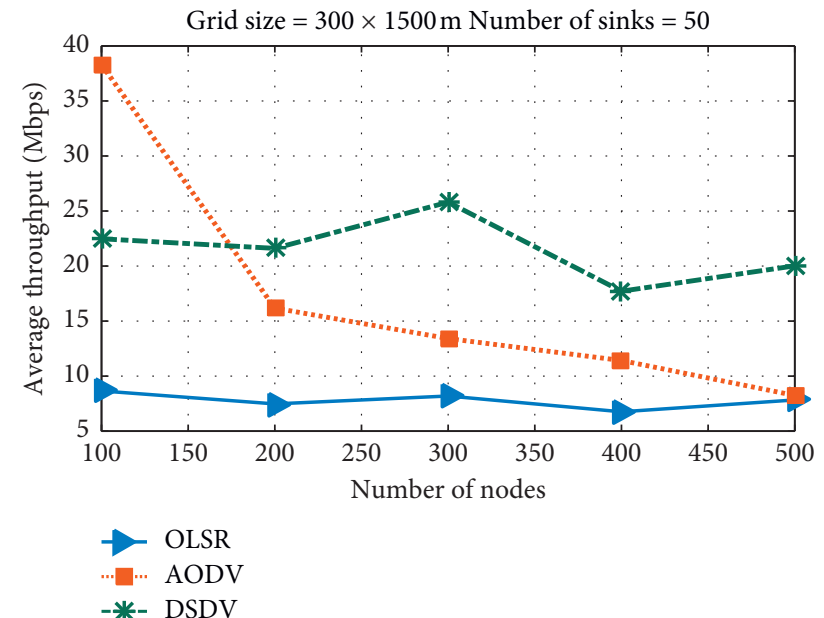

(a)

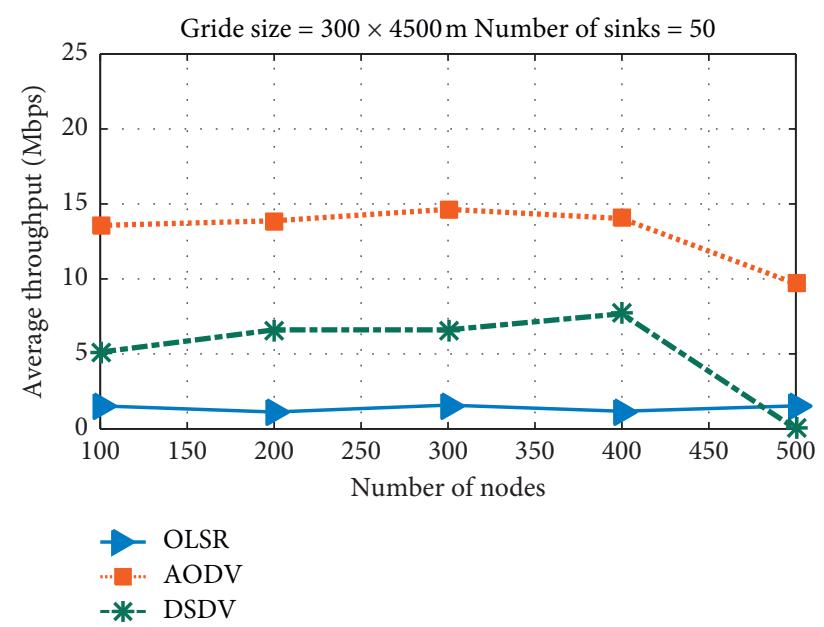

(c)

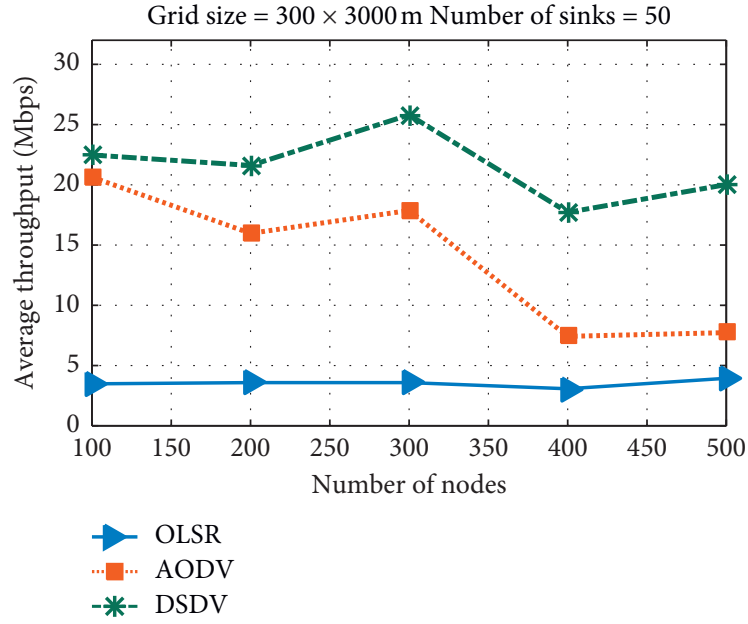

(b)

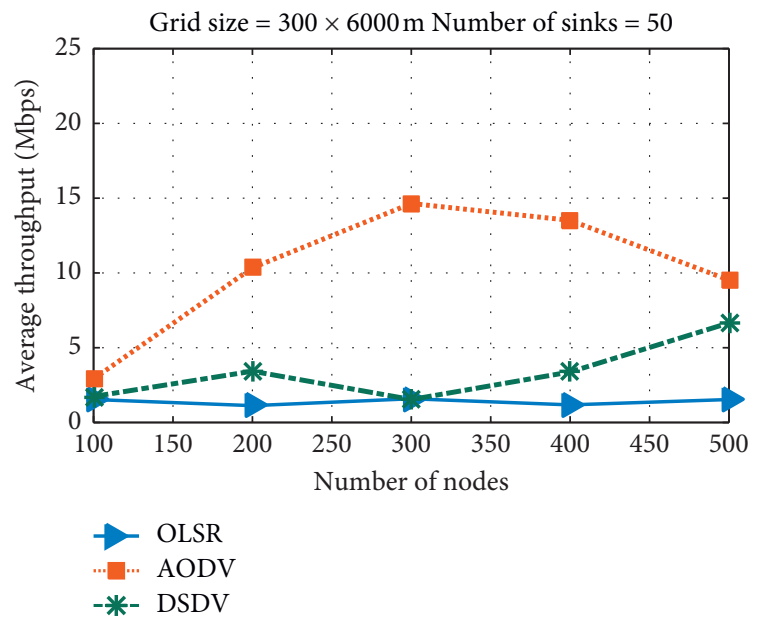

(d)

FIgURE 6: Average throughput in traditional VANET with no. of sinks $=50$.

Grid size $=300 \times 1500 \mathrm{~m}$ Number of sinks $=25$

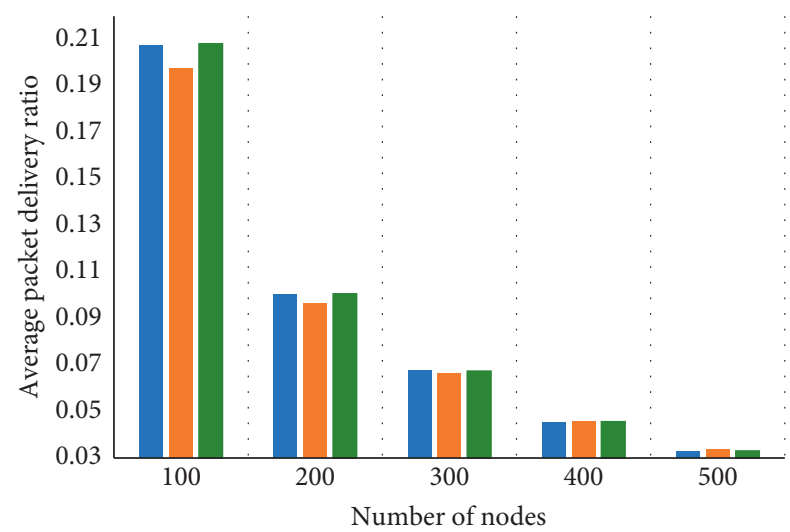

- OLSR

- AODV

- DSDV
Grid size $=300 \times 3000 \mathrm{~m}$ Number of sinks $=25$

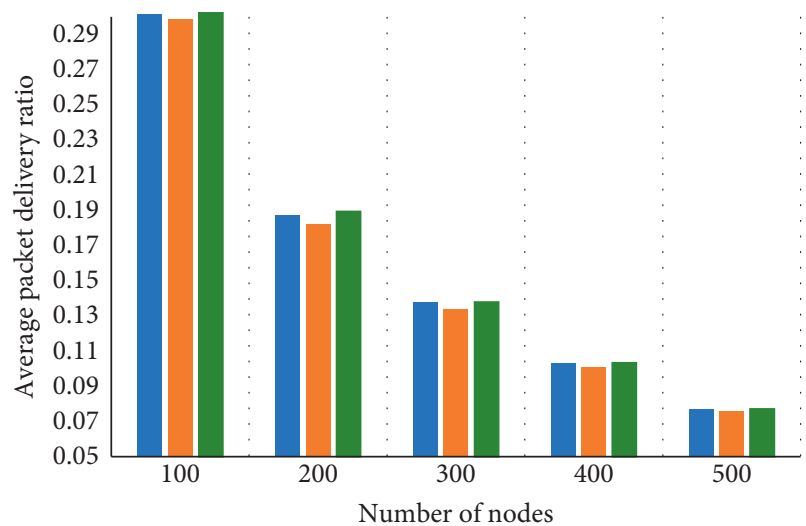

- OLSR

- AODV

- DSDV

(a)

FIgure 7: Continued. 


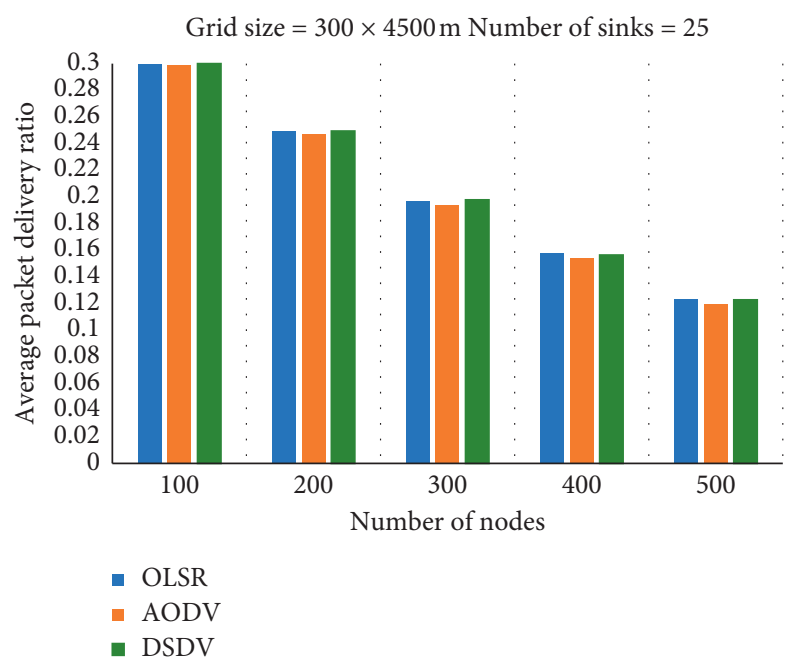

(c)

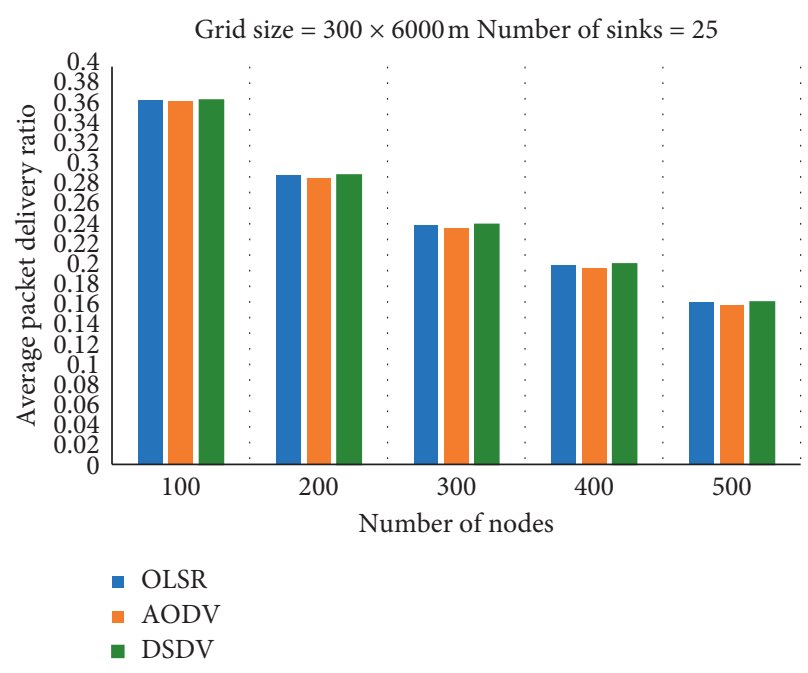

(d)

Figure 7: Average packet drop ratio in traditional VANET with no. of sinks $=25$.

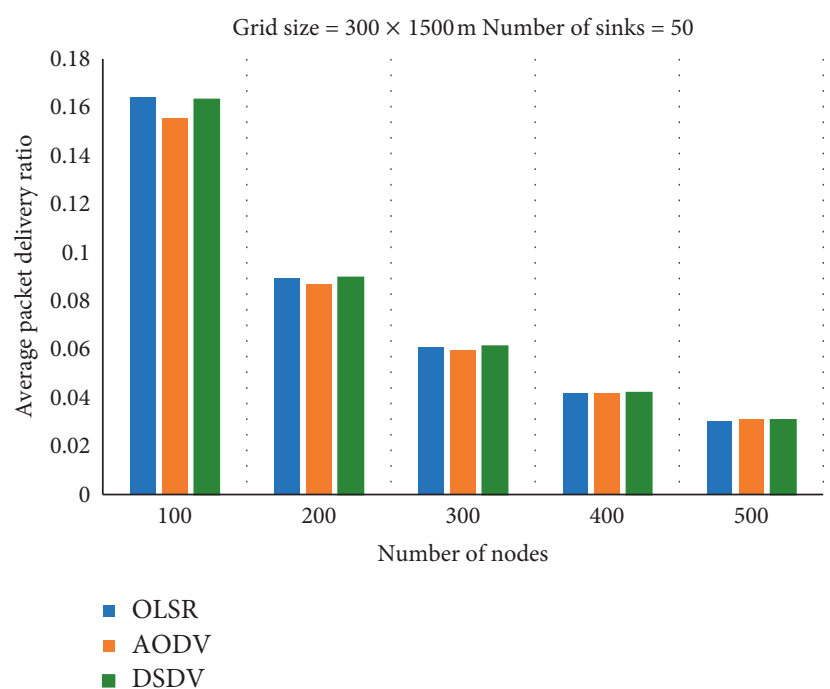

(a)

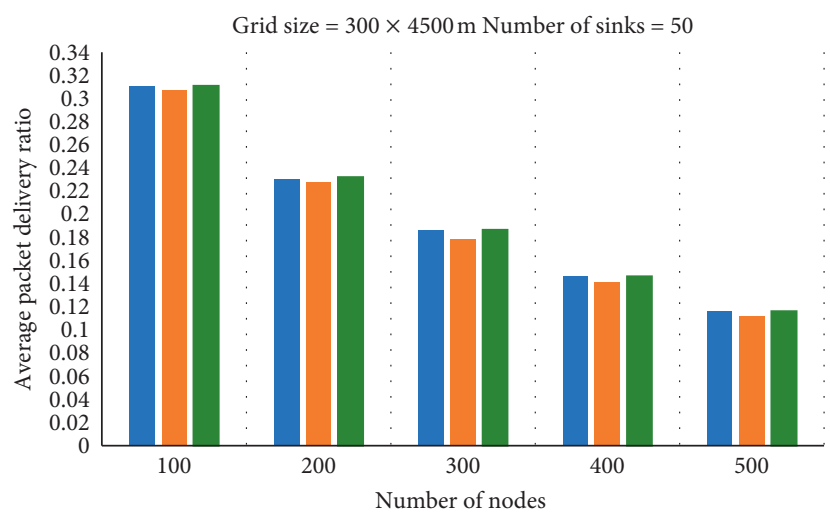

- OLSR

- AODV

- DSDV

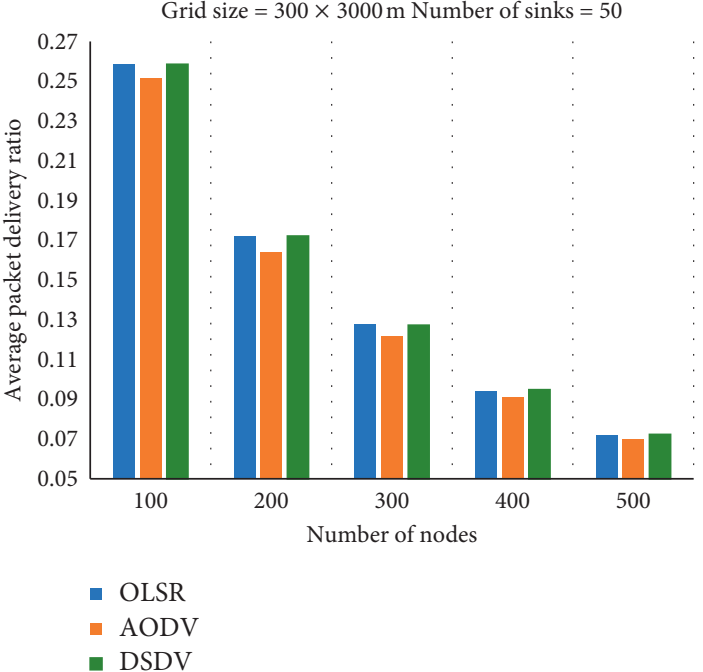

(b)

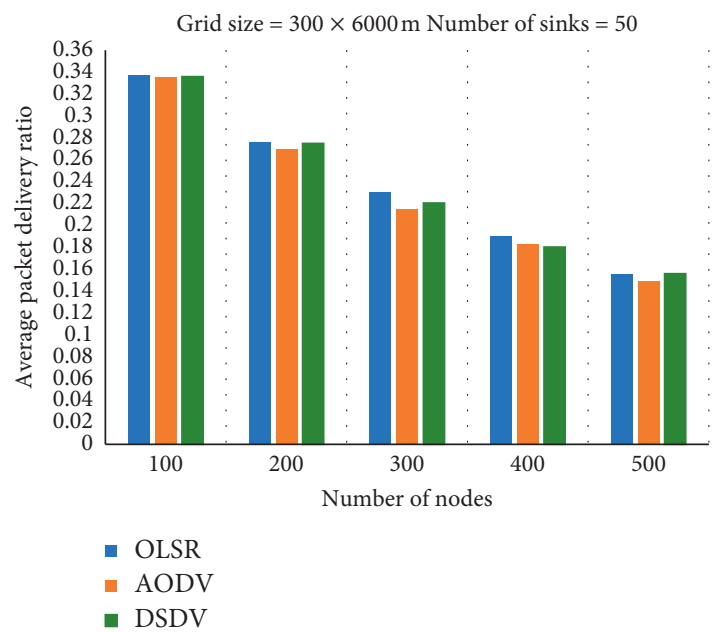

(d)

Figure 8: Average packet drop ratio in traditional VANET with no. of sinks $=50$. 


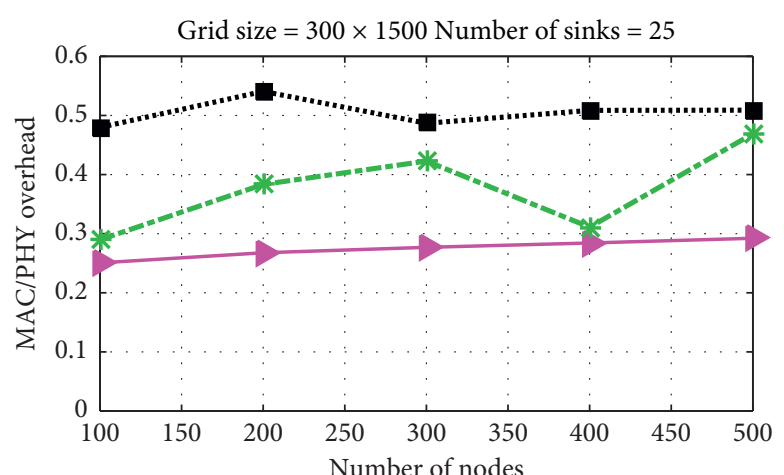

- DA-OLSR
...... DA-AODV
- 2 - DA-DSDV

(a)

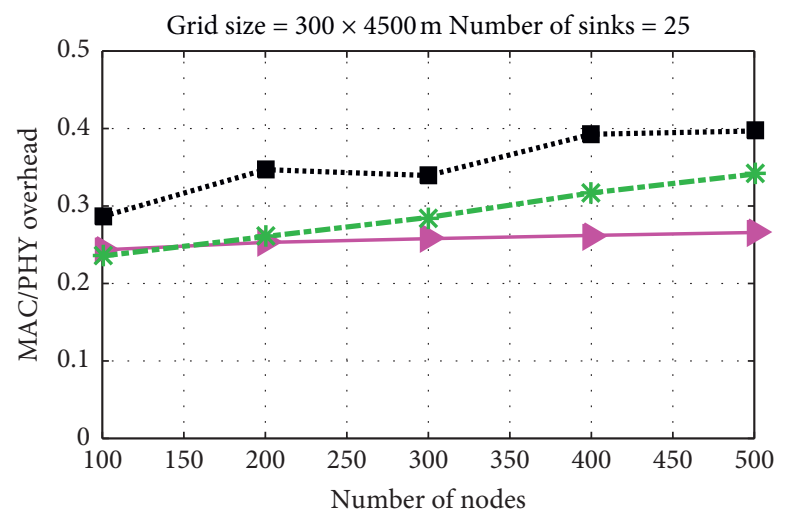

DA-OLSR

DA-AODV

DA-DSDV

(c)

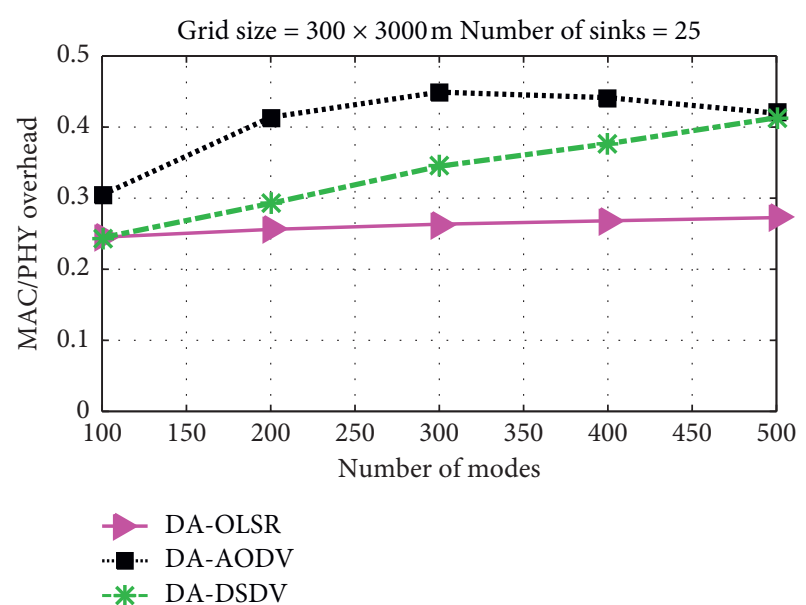

(b)

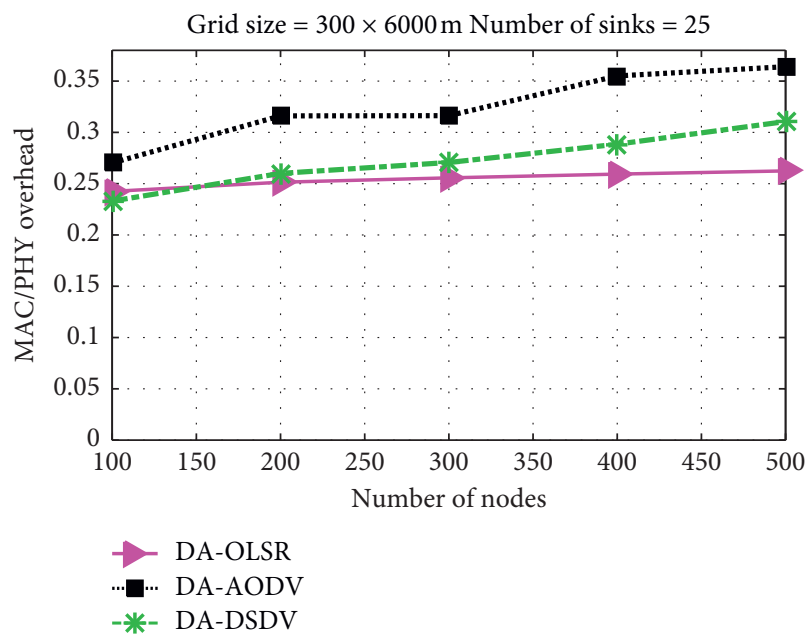

(d)

FIgURe 9: MAC/PHY overhead with drone assistance and no. of sinks $=25$.

Figure 9(b) represents results for drone-assisted protocol within grid size of $300 \times 3000 \mathrm{~m}$. It is clear from Figure 10 that MAC/PHY overhead for DA-OSLR ascends slightly with the ascending number of nodes. MAC/PHY overhead for DA-DSDV ascends when the number of nodes ascends. MAC/PHY overhead for DA-AODV ascends when the number of nodes ascends from 100 to 300 , and it decreases when we have 300 to 500 vehicular nodes.

It is clear from Figure 9(c) that MAC/PHY overhead for DA-OLSR ascends with the ascending number of nodes. MAC/PHY overhead for DA-DSDV increases when the number of nodes increases. MAC/PHY overhead for DAAODV ascends when the number of nodes ascends from 100 to 200 , and it becomes constant when the number of nodes ascends from 200 to 300 . Its performance is enhanced when the number of nodes ascends from 300 to 400 and becomes constant after that. Here, the grid size is $300 \times 4500 \mathrm{~m}$.

Figure 9(d) shows results simulated in a grid size of $300 \times 6000 \mathrm{~m}$. The MAC/PHY overhead for DA-OLSR ascends linearly with the ascending number of nodes. MAC/PHY overhead for DA-DSDV increases when the number of nodes increases. It can be seen easily that MAC/PHY overhead for DA-AODV is higher than that of all the other protocols, whatever the number of nodes we have.

5.2.2. MAC/PHY Overhead with 50 Sink Nodes. The number of sink nodes is kept constant for all the scenarios shown in Figure 10. The grid size increases from $300 \times 1500 \mathrm{~m}$ to $300 \times 6000 \mathrm{~m}$ whereas the number of vehicular nodes is from 100 to 500. The protocols involved in the simulations are OLSR, AODV, and DSDV with the assistance of aerial nodes. Figure 10(a) shows the simulated results generated for grid size $300 \times 1500 \mathrm{~m}$. The performance of DA-OLSR decreases with a slight change at every point throughout the simulations. The DA-DSDV performance is low at the start and up to node 300 , after that its performance is neither 

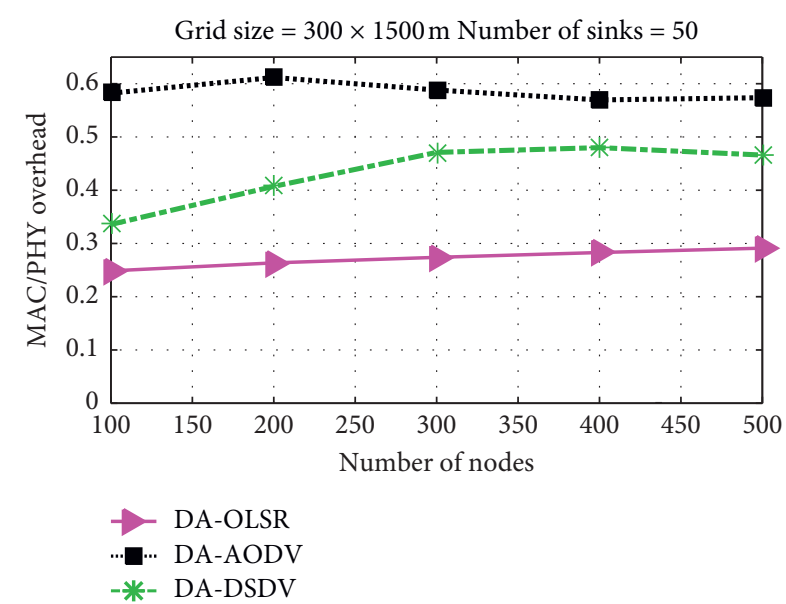

(a)

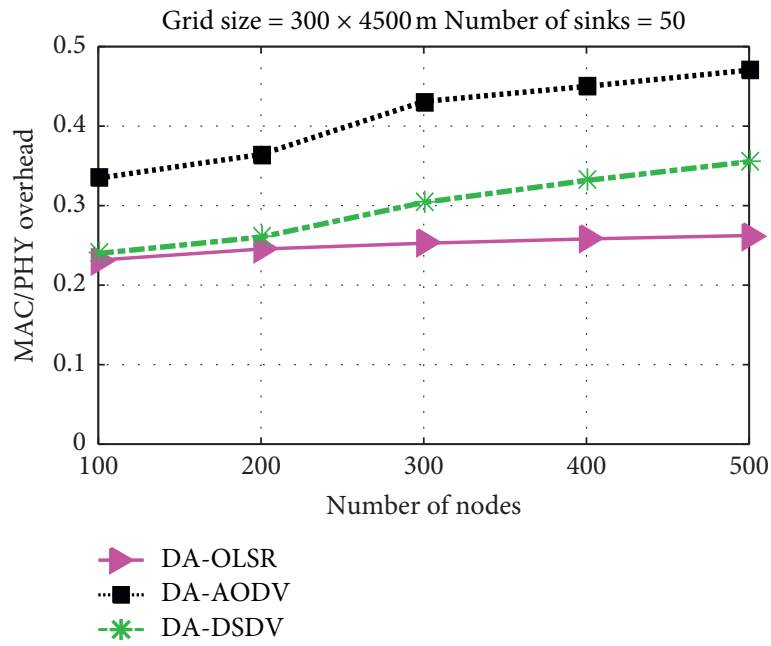

(c)

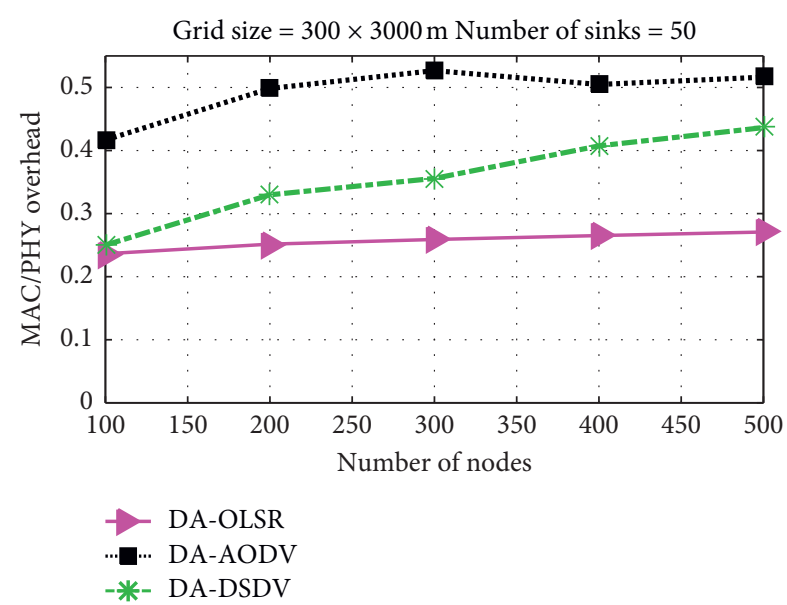

(b)

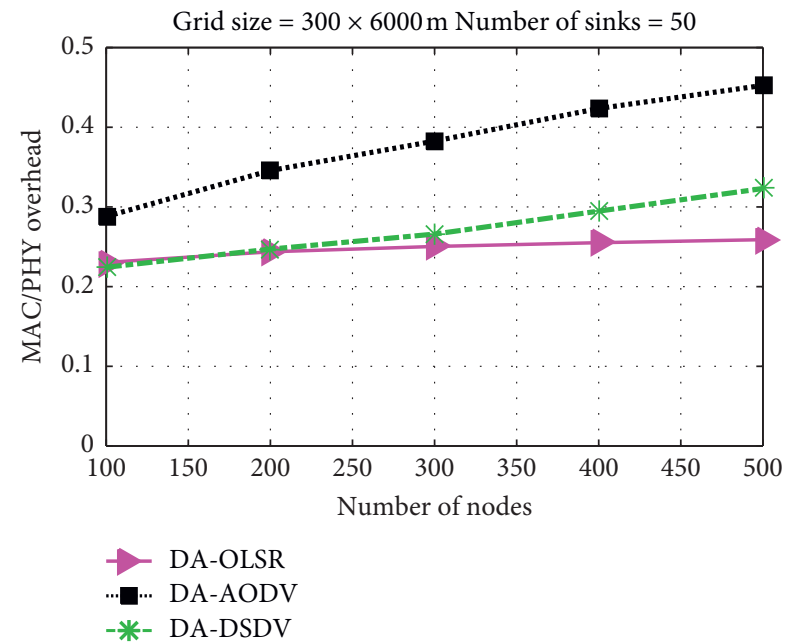

(d)

FIGURE 10: MAC/PHY overhead with drone assistance and no. of sinks $=50$.

increasing nor decreasing, but as the number of nodes reaches 400, the performance of DA-DSDV starts to enhance. From Figure 10(b), we can conclude that the performance of DA-OLSR is the best one while the DA-AODV has the least performance within grid size of $300 \times 3000 \mathrm{~m}$. The DA-DSDV lies between the other two protocols.

Results simulated in grid sizes of $300 \times 4500 \mathrm{~m}$ and $300 \times 6000 \mathrm{~m}$ are shown in Figures $10(\mathrm{c})$ and $10(\mathrm{~d})$, respectively. We can see clearly that the DA-AODV protocol has the highest MAC/PHY overhead throughout the simulations as compared to the other two protocols, while DAOLSR has the least MAC/PHY overhead. In the case of DADSDV, MAC/PHY overhead increases with the increase in the number of vehicular nodes.

5.2.3. Average Throughput with 25 Sink Nodes. The average throughput for DA-OLSR, DA-AODV, and DA-DSDV calculated with 25 sink nodes is represented in Figure 11. The size of the grid increases from $300 \times 1500 \mathrm{~m}$ to $300 \times 6000 \mathrm{~m}$ with an interval of $1500 \mathrm{~m}$ each time. The number of vehicular nodes ranges from 100 to 500. Figure 11(a) shows that the average throughput for DA-OLSR increases as nodes ascends from 100 to 200 and then remains constant with vehicular nodes from 200 to 400 . It shows a gradual decrease with nodes from 450 to 500 . The second protocol, DA-AODV, shows excellent throughput on the first 100 nodes but then shows a rapid decrease with nodes from 100 to 500. In the case of DA-DSDV, average throughput increases as the number of nodes increases from 100 to 200 and then remains constant with nodes from 200 to 300; after that, its performance gets worse with nodes from 300 to 400 . There is an increase once again in the throughput as the number of nodes reaches from 400 to 500 .

From Figure 11(b), it is clear that the DA-OLSR shows the minimum average throughput as compared to the other ones. It remains constant with nodes from 100 to 200, and then its throughput decreases as the number of nodes ascends from 200 to 400 . Its performance degrades with nodes from 400 to 500. DA-AODV shows a high average 


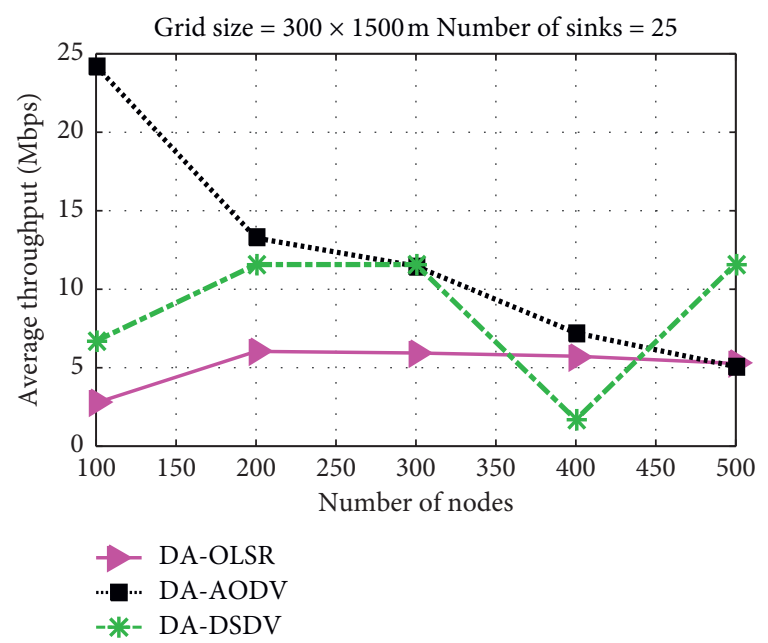

(a)

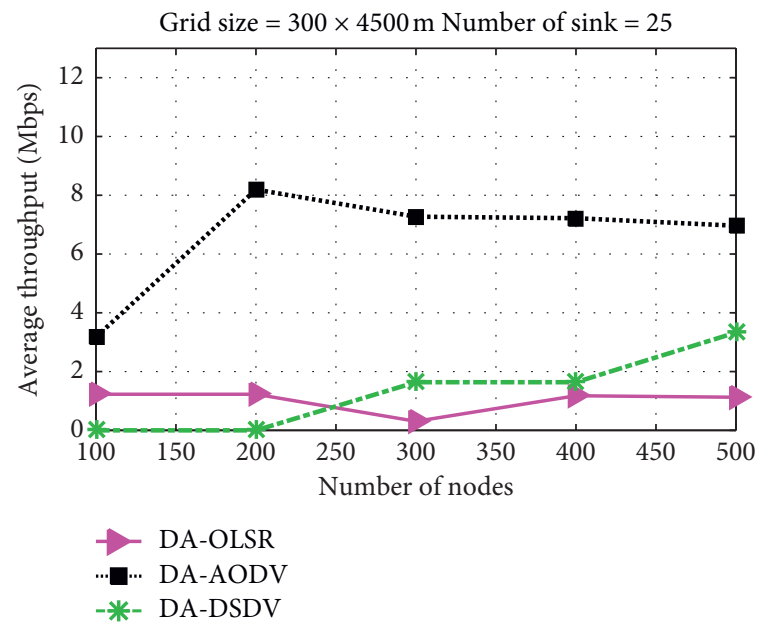

(c)

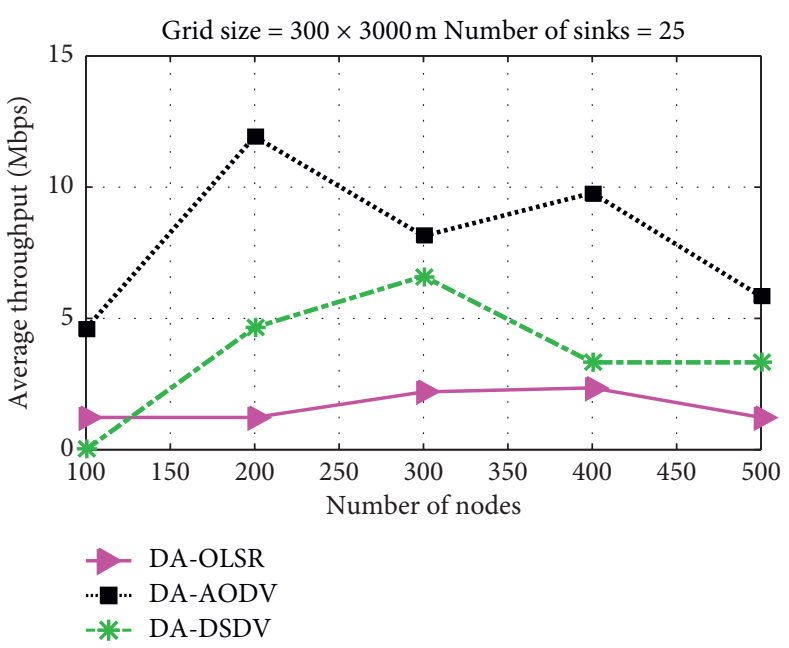

(b)

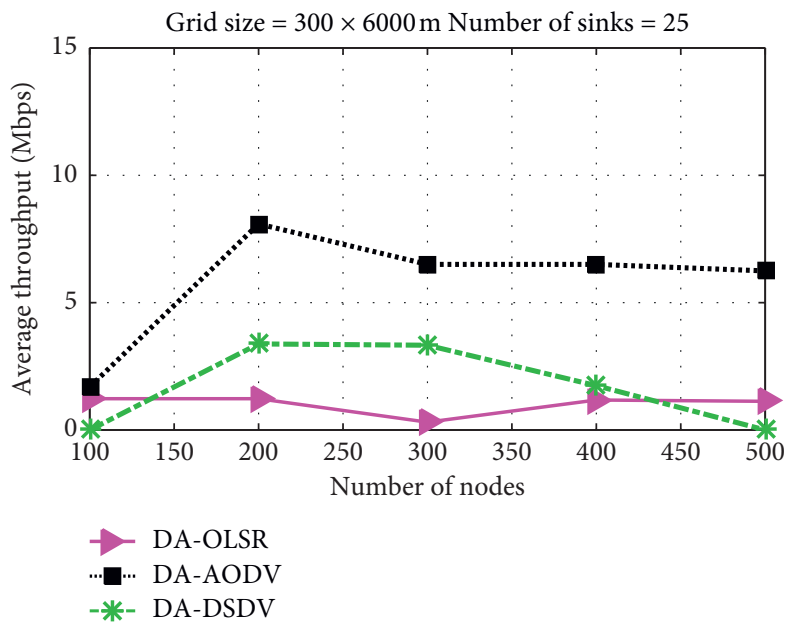

(d)

FIgURE 11: Average throughput with drone assistance and no. of sinks $=25$.

throughput as compared to the other ones. It increases rapidly as the number of nodes ascends from 100 to 200 , and then its starts decreasing with nodes from 200 to 300 . Then, again it rises with nodes from 300 to 400, and after that it again starts decreasing as the number of nodes ascends from 400 to 500. DA-DSDV shows an increase as the number of nodes ascends from 100 to 300, and then it decreases with nodes from 300 to 400; after that, it remains constant with nodes from 400 to 500 .

As shown in Figure 11(c) DA-OLSR shows minimum throughput throughout, as it remains constant with nodes from 100 to 200, and then it decreases as the nodes ascend and then rises with nodes from 300 to 400 . Then, it remains constant again with nodes from 400 to 500. DA-AODV shows good throughput as it increases as the number of nodes ascends, and then it gradually decreases with nodes from 200 to 300; after that, it almost remains constant from 300 to 500 nodes. DA-DSDV shows minimum throughput with nodes from 100 to 200 . The performance of DA-DSDV is enhanced as we increase the number of vehicular nodes from 200 to 500 .
Figure 11(d) demonstrates the average throughput in grid size of $300 \times 4500 \mathrm{~m}$. As can be seen, DA-OLSR remains constant but has less throughput at the start. Its throughput decreases as nodes ascend, and then again it starts rising with nodes from 300 to 400 . The throughput is constant with nodes from 400 to 500. DA-AODV shows a rapid increase in throughput as nodes ascend from 100 to 200, and then its performance degrades with nodes from 200 to 300 . The average throughput has a constant value with nodes from 300 to 500 . DA-DSDV shows an increase in throughput as nodes ascend from 100 to 200; then, with nodes from 200 to 300, it remains constant; and after that it decreases with nodes from 300 to 500 .

5.2.4. Average Throughput with 50 Sink Nodes. For the scenario shown in Figures 12(a)-12(d), we have the number of sink nodes equal to 50 . The grid size increases from $300 \times 1500 \mathrm{~m}$ to $300 \times 6000 \mathrm{~m}$. We have taken the vehicular nodes from 100 to 500 . 


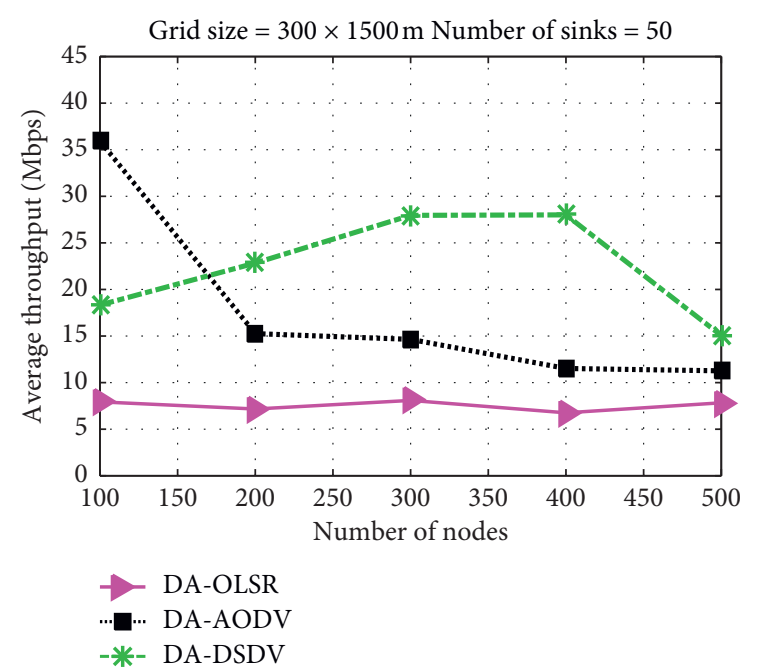

(a)

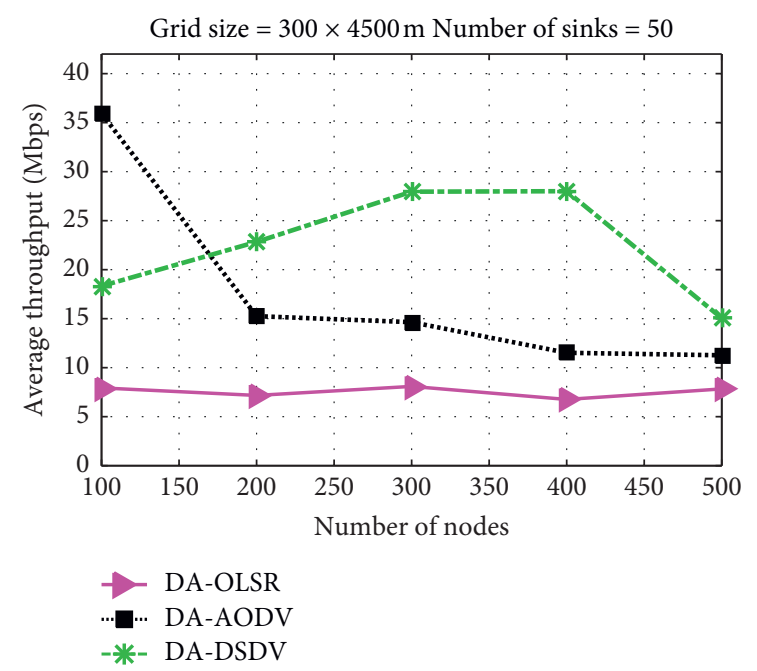

(c)

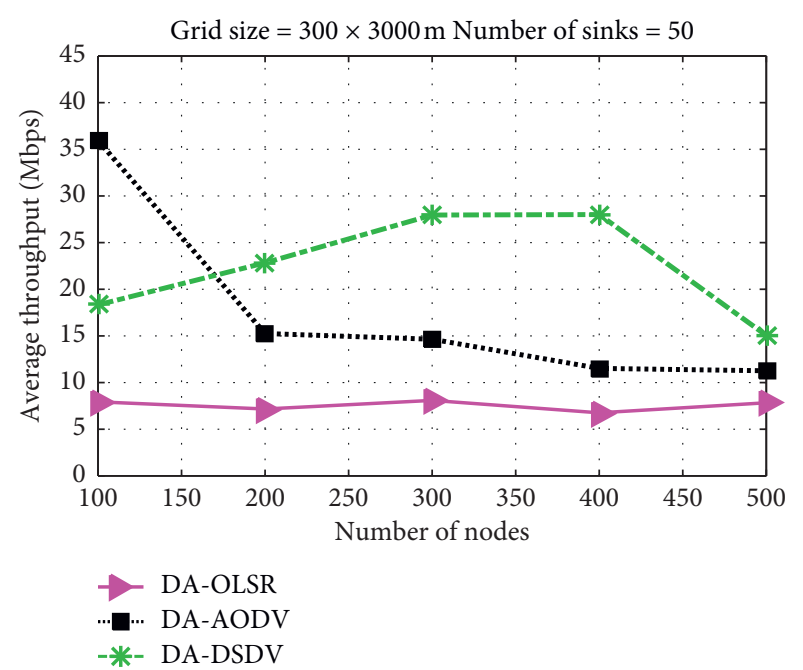

(b)

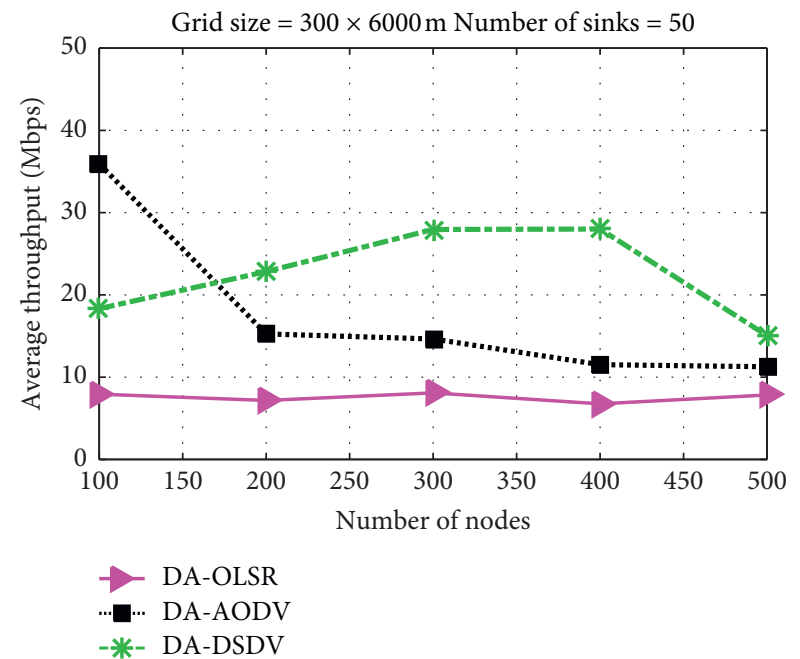

(d)

FIGURE 12: Average throughput with drone assistance and no. of sinks $=50$.

Figures $12(\mathrm{a})$ and $12(\mathrm{~b})$ show the average throughput within grid sizes of $300 \times 1500 \mathrm{~m}$ and $300 \times 3000 \mathrm{~m}$. Average throughput in the case of DA-OLSR remains constant as the number of nodes increases with a little rise and fall with nodes from 100 to 500. DA-AODV has the highest performance at 100 nodes, and then it decreases as the number of nodes increases up to 500. DA-DSDV performance increases as the number of nodes ascend from 100 to 400 and then remains constant with nodes from 300 to 400 . With nodes from 400 to 500 , the throughput decreases.

Figures $12(\mathrm{c})$ and $12(\mathrm{~d})$ represent average throughput in grid sizes of $300 \times 4500 \mathrm{~m}$ and $300 \times 6000 \mathrm{~m}$, respectively. DA-OLSR almost remains constant with a little increase and decrease as the nodes ascend. DA-AODV decreases rapidly as the number of vehicular nodes decreases from 100 to 500 . DA-DSDV shows an increase as the number of nodes increases from 100 to 300 , and then it shows a constant value with nodes between 300 and 400 . With nodes from 400 to 500 , the average throughput decreases.
5.2.5. Average Packet Delivery Ratio with 25 Sink Nodes. For the scenario shown in Figures 13(a)-13(d), we have the number of sink nodes equal to 25 . The grid size increases from $300 \times 1500 \mathrm{~m}$ to $300 \times 6000 \mathrm{~m}$. We have taken the vehicular nodes from 100 to 500. Figures 13(a)-13(d) show the average packet delivery ratio of the three protocols, DAOLSR, DA-AODV, and DA-DSDV. In grid size of $300 \times 1500 \mathrm{~m}$, the performance of DA-AODV is less than that of the other two protocols, whereas DA-OLSR and DADSDV show very close results. At 100 nodes, the three protocols give the highest average packet delivery ratio, but as we ascend the number of vehicular nodes, there is a decrease in average packet delivery ratio in all four grid sizes. However, it is obvious from Figures 13(a)-13(d) that the three protocols are giving a better average packet delivery ratio as we ascend the grid size each time. The performance of the three protocols is better at grid size of $300 \times 6000 \mathrm{~m}$ than that at $300 \times 1500 \mathrm{~m}$. Moreover, the DA-DSDV surpasses the DA-OLSR and DSDV at vehicular nodes 100 to 
Grid size $=300 \times 1500 \mathrm{~m}$ Number of sinks $=25$

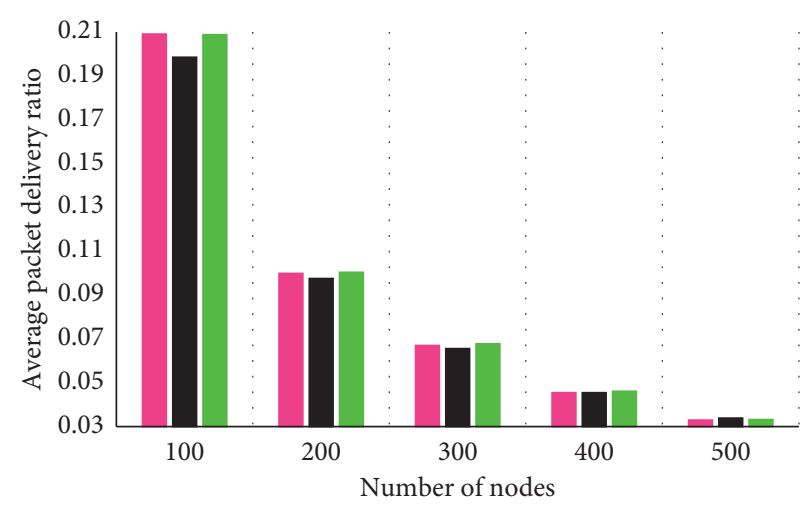

- DA-OLSR

- DA-AODV

- DA-DSDV

(a)

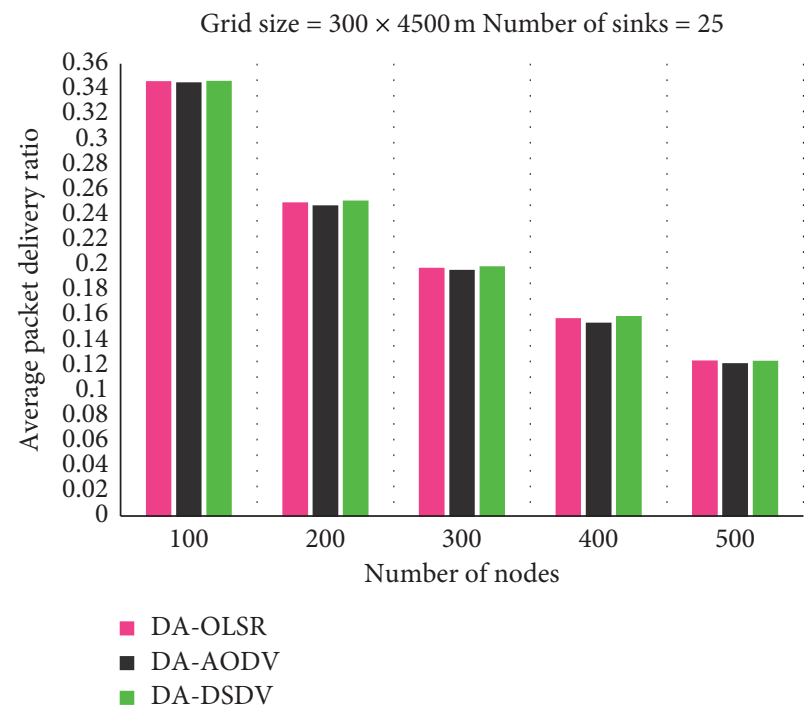

(c)

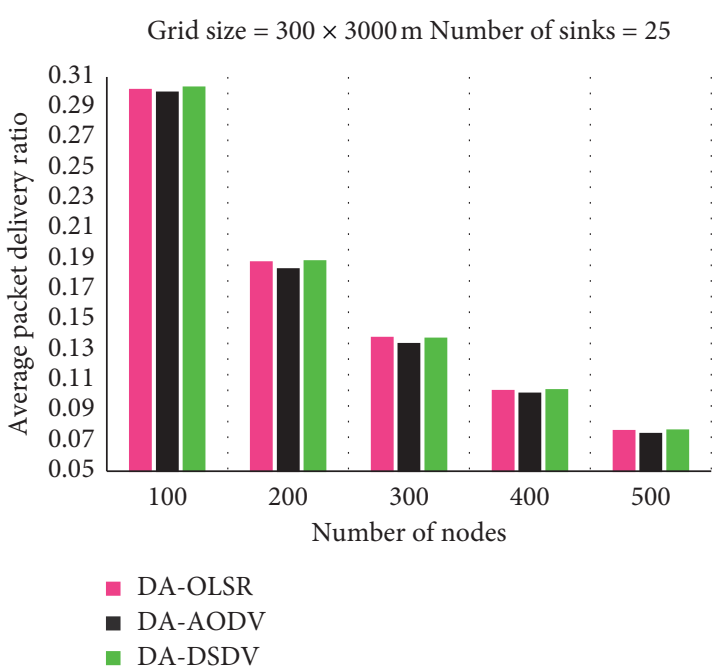

(b)

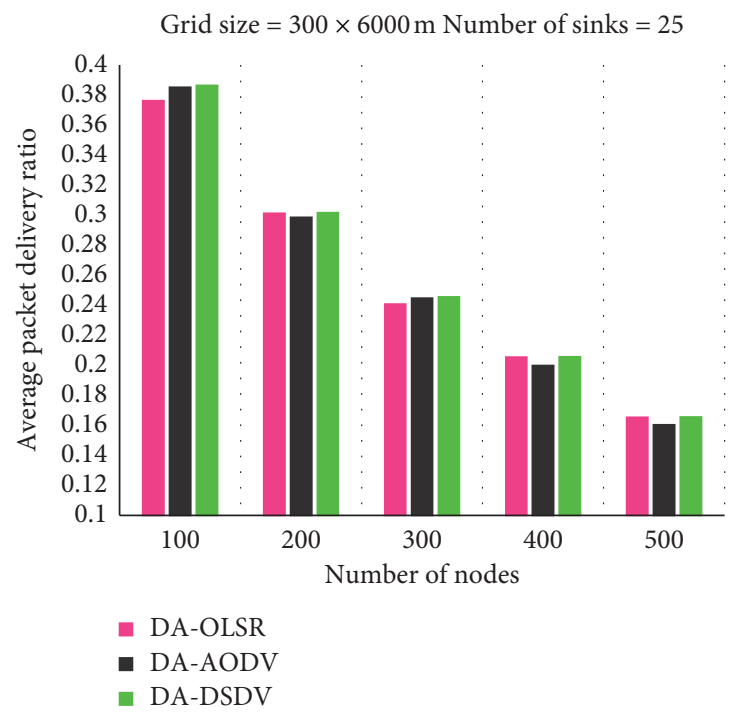

(d)

FIGURE 13: Average packet delivery ratio with drone assistance and no. of sinks $=25$.

500. Thus, we can conclude that average packet delivery ratio increases as we increase the grid size.

\subsubsection{Average Packet Delivery Ratio with 50 Sink Nodes.} For the scenario shown in Figures 14(a)-14(d), we have the number of sink nodes equal to 50 . The grid size increases from $300 \times 1500 \mathrm{~m}$ to $300 \times 6000 \mathrm{~m}$. We have taken the vehicular nodes from 100 to 500. Figures 15 (a)-15(d) show the average packet delivery ratio of the three protocols, DAOLSR, DA-AODV, and DA-DSDV. In grid size of $300 \times 1500 \mathrm{~m}$, the performance of DA-AODV is less than that of the other two protocols, whereas DA-OLSR and DADSDV show very close results. At 100 nodes, the three protocols give the highest average packet delivery ratio, but as we ascend the number of vehicular nodes, there is a decrease in average packet delivery ratio in all four grid sizes. However, it is obvious from Figures 14(a)-14(d) that the three protocols are giving a better average packet delivery ratio as we ascend the grid size each time. The performance of the three protocols is better at grid size of $300 \times 6000 \mathrm{~m}$ than that at $300 \times 1500 \mathrm{~m}$. Furthermore, the DA-DSDV surpasses the DA-OLSR and DSDV at vehicular nodes 100 to 500. Consequently, we can conclude that average packet delivery ratio increases as we increase the grid size.

\section{Comparative Analysis of Traditional VANET and Drone-Assisted VANET}

For detailed analysis to figure out which scenario is better for the IoV environment, we have combined the traditional VANET routing protocols and drone-assisted VAENT protocols. These combined graphs will help in a deep insight into the conducted simulations.

6.1. MAC/PHY Overhead with 25 Sink Nodes. Figure 15 illustrates that, for all the grid sizes, the OLSR and DA-OLSR have the least MAC/PHY overhead, whereas the highest 


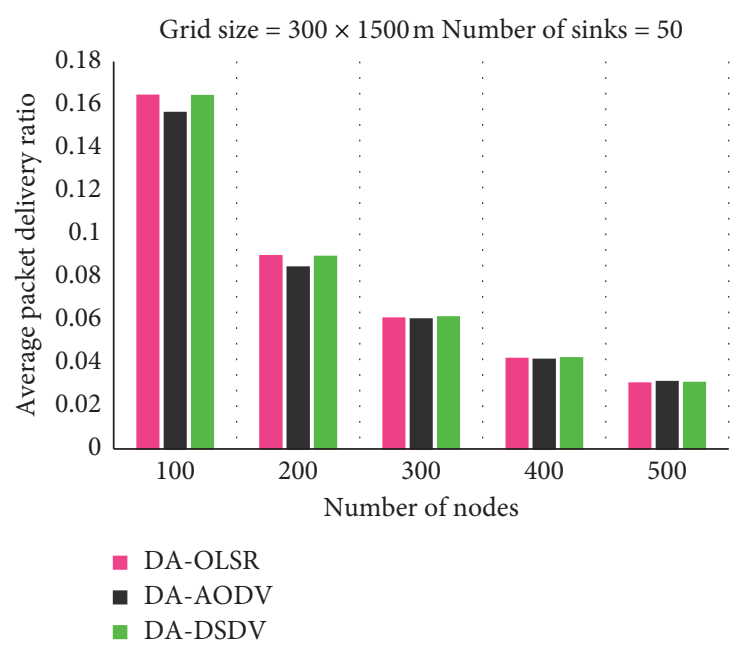

(a)

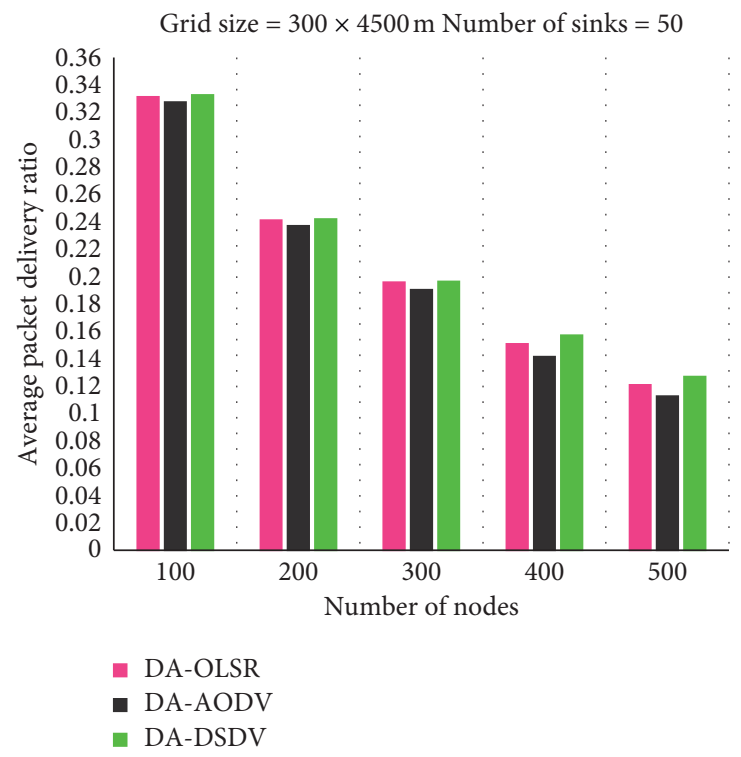

(c)

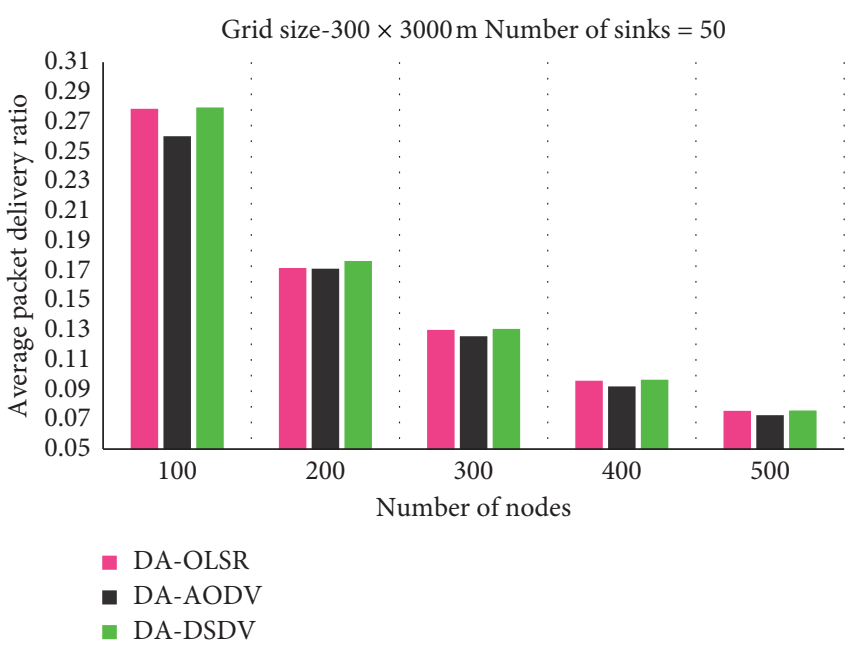

(b)

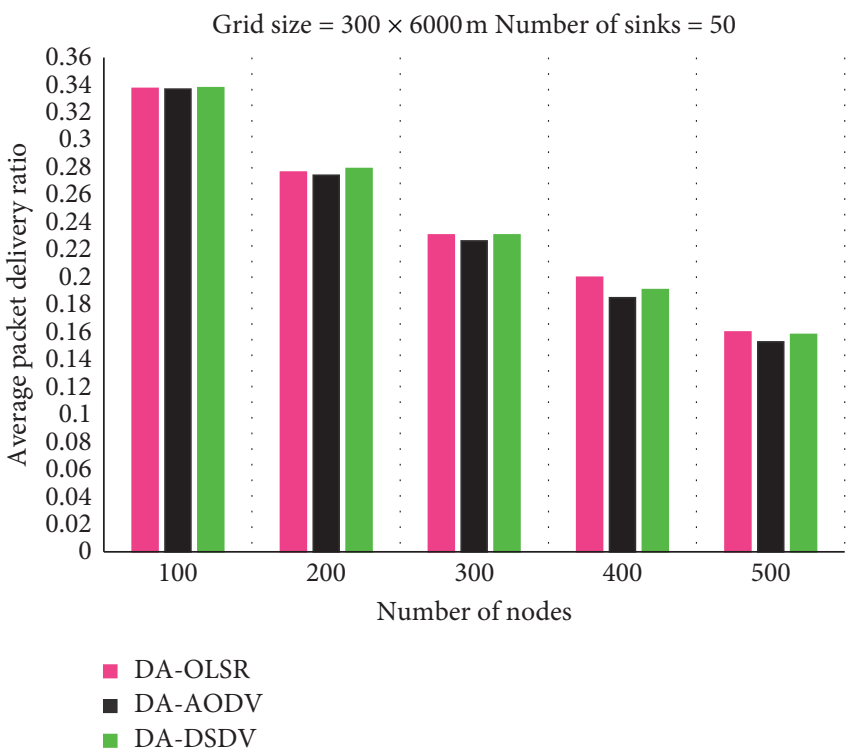

(d)

FIgURE 14: Average packet delivery with drone assistance and no. of sinks $=50$.

MAC/PHY overhead is depicted in AODV, and the rest of the protocols lie between both. However, if we compare the performance of traditional VANET routing protocols to that of the drone-assisted ones, it will be clear that drone-assisted protocols show less MAC/PHY overhead. When we have a smaller grid, the MAC/PHY overhead for all the six protocols has greater values, but as we ascend towards a bigger grid, this MAC/PHY overhead decreases. The results presented in Figures 15(a)-15(d) are for the same number of sink nodes, i.e., 25, and vehicular nodes for the presented four graphs are from 100 to 500 . The grid size is initially $300 \times 1500 \mathrm{~m}$ and reaches up to $300 \times 6000 \mathrm{~m}$ with a constant interval of $1500 \mathrm{~m}$ along the $y$-axis.

6.2. MAC/PHY Overhead with 50 Sink Nodes. Figure 16 clearly shows that, for all grid sizes, the OLSR and DAOLSR have the least MAC/PHY overhead except for
$300 \times 6000 \mathrm{~m}$ where DA-OLSR has less MAC/PHY overhead even compared to OLSR. This means that at a bigger grid size the performance of drone-assisted OLSR is far better than that of the rest of the protocols. On the other hand, the highest MAC/PHY overhead is depicted in AODV. The rest of the protocols lie between DA-OLSR and AODV. When we compare the performance of traditional VANET routing protocols to that of the drone-assisted ones, it becomes clear that drone-assisted protocols show less MAC/PHY overhead for most of the cases. When we have a smaller grid, the MAC/PHY overhead for all the six protocols has greater values, but as we ascend towards a bigger grid, this MAC/ $\mathrm{PHY}$ overhead decreases. It can be concluded from Figure 16 that, for a bigger grid size like $300 \times 6000 \mathrm{~m}$, the droneassisted protocols outperform the traditional ones. The results presented in Figures 16(a)-16(d) are for the same number of sink nodes, i.e., 50 , and vehicular nodes for the presented four graphs are from 100 to 500 . The grid size is 


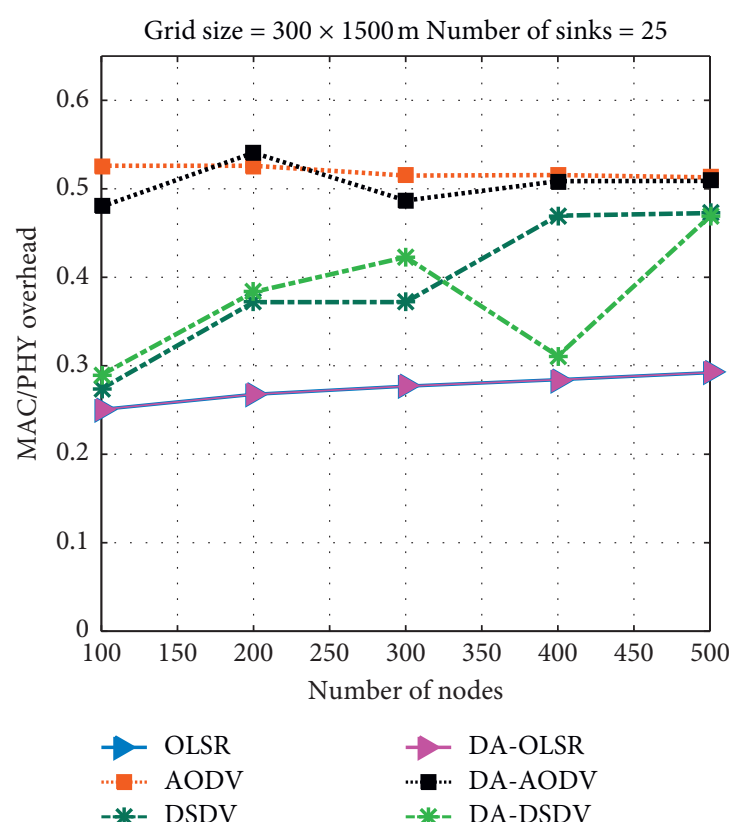

(a)

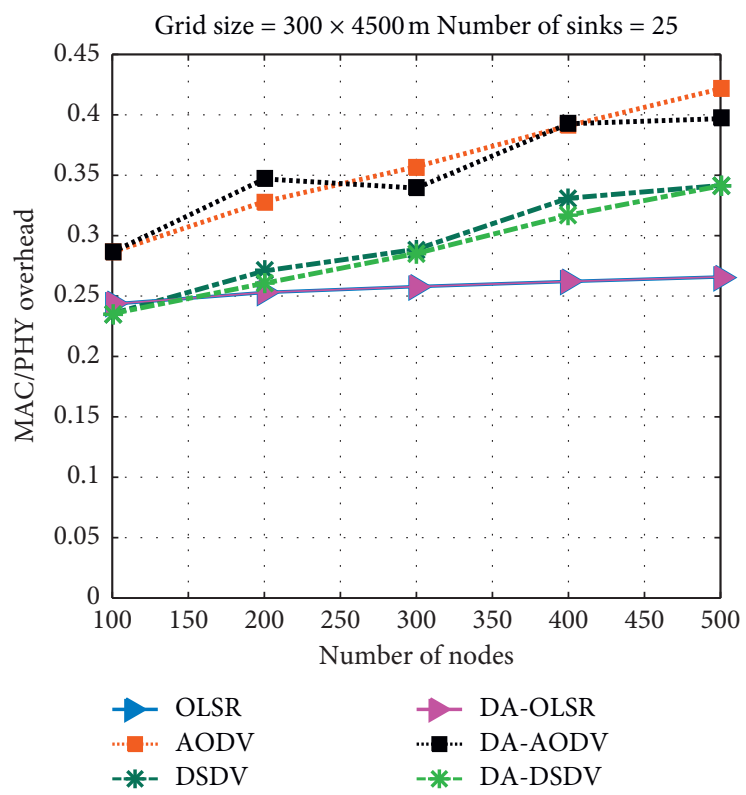

(c)

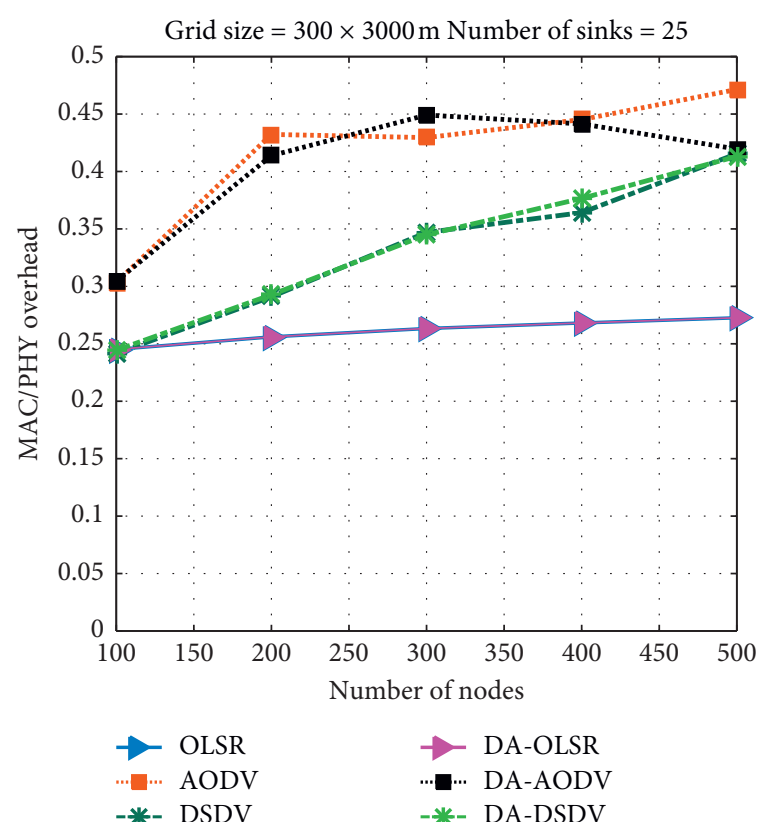

(b)

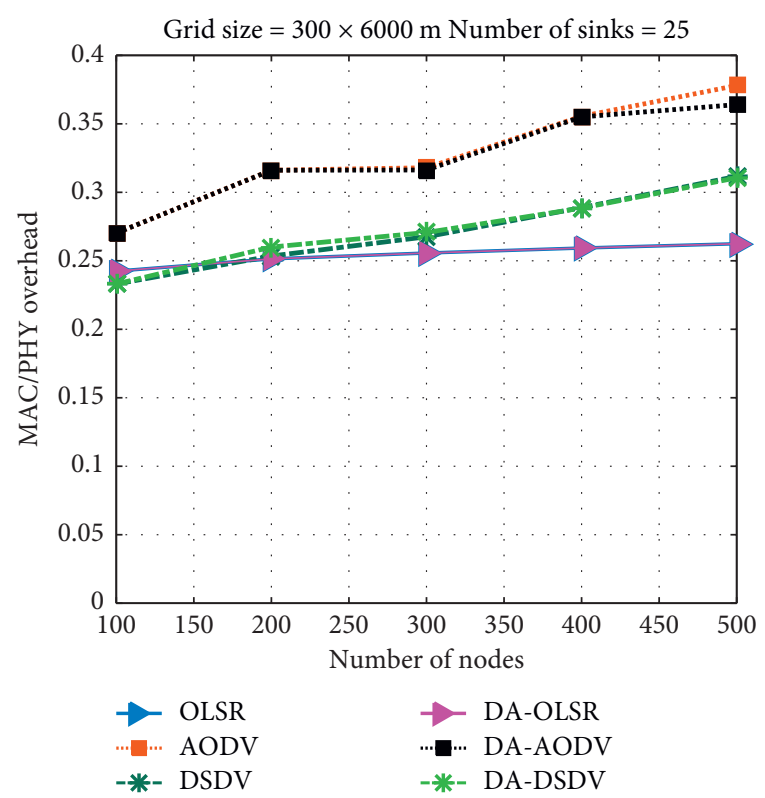

(d)

FIGURE 15: Comparison of MAC/PHY overhead of scenarios a and b with 25 sinks.

initially $300 \times 1500 \mathrm{~m}$ and reaches up to $300 \times 6000 \mathrm{~m}$ with a constant interval of $1500 \mathrm{~m}$ along the $y$-axis. One more thing to be noted is that the greater the number of sink nodes is, the higher the performance of protocols will be. As can be seen from Figures 15 and 16, all the protocols have better performance when we have a number of sinks $=50$, especially the drone-assisted protocols.

6.3. Average Throughput with 25 Sink Nodes. Figure 17 shows that, for all the grid sizes, the OLSR and DA-OLSR have the least average throughput, whereas the highest average throughput is depicted in DA-AODV when we have the least number of vehicular nodes, and the rest of the protocols lie between both. If we compare the performance of traditional VANET routing protocols to that of the drone-assisted ones, it will be clear that drone-assisted protocols show less throughput when we have a greater number of nodes. When we have a smaller grid, the MAC/PHY overhead for all the six protocols has greater values, but as we ascend towards a bigger grid, this average throughput decreases. This is because of the dissemination of vehicular nodes at a great distance due to an increase in grid size. The vehicular nodes are unable to communicate with each other, hence resulting 


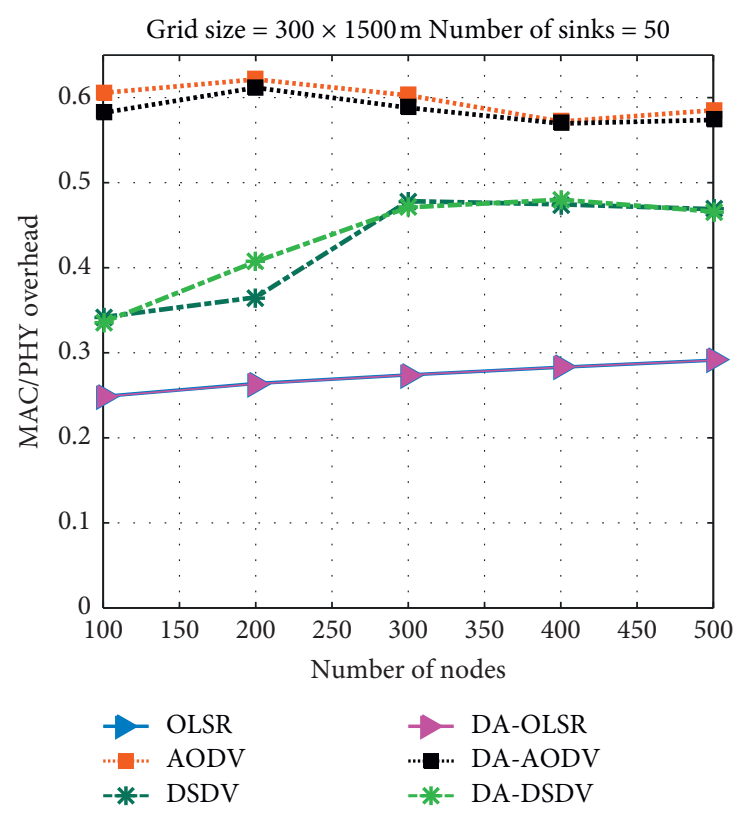

(a)

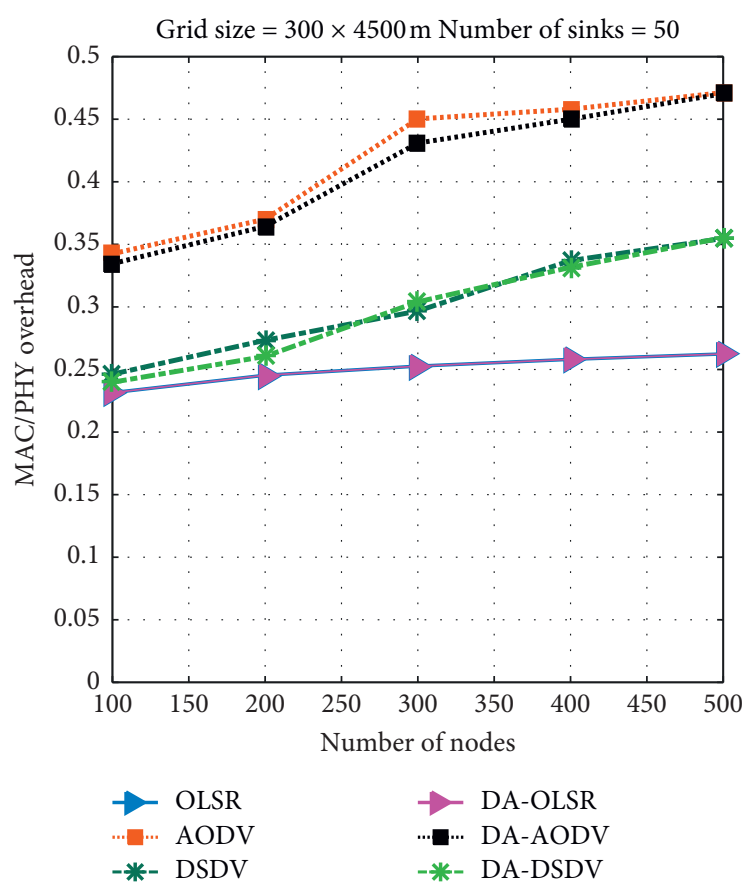

(c)

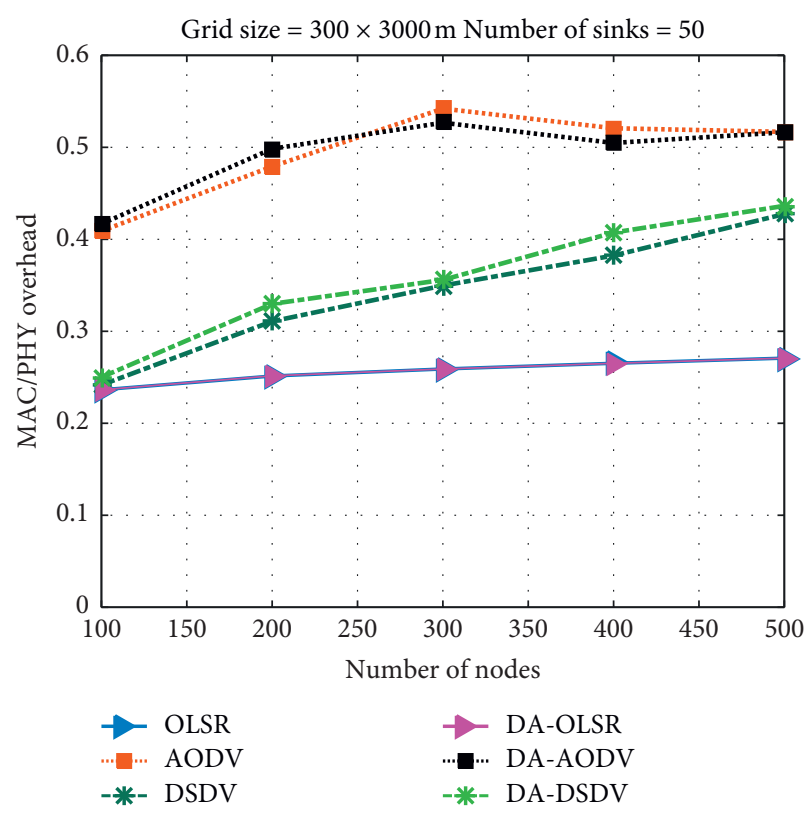

(b)

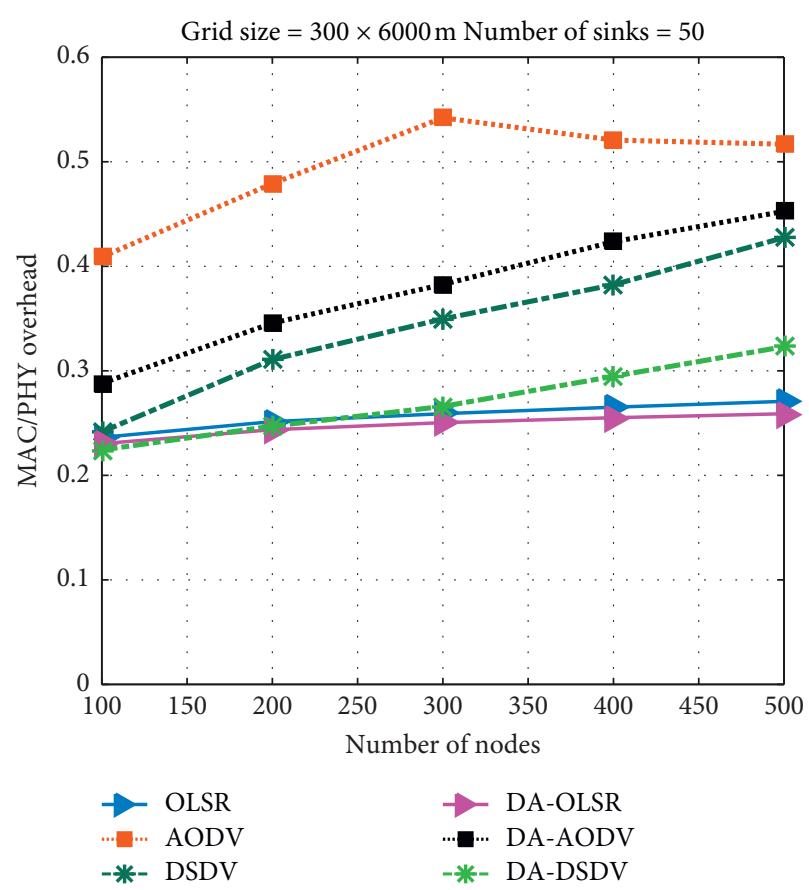

(d)

FIgURE 16: Comparison of MAC/PHY overhead of scenarios a and b with 50 sinks.

in less throughput. The results presented in Figures 17(a)17 (d) are for the same number of sink nodes, i.e., 25, and vehicular nodes for the presented four graphs are from 100 to 500 . The grid size is initially $300 \times 1500 \mathrm{~m}$ and reaches up to $300 \times 6000 \mathrm{~m}$ with a constant interval of $1500 \mathrm{~m}$ along the $y$-axis.
6.4. Average Throughput with 50 Sink Nodes. Figure 18 clearly shows that, for all grid sizes, the OLSR and DAOLSR have the least average throughput, whereas the highest average throughput is shown by DSDV in smaller grid sizes, but when we have larger grid sizes, the performance of DAAODV is better for a greater number of nodes. The rest of 


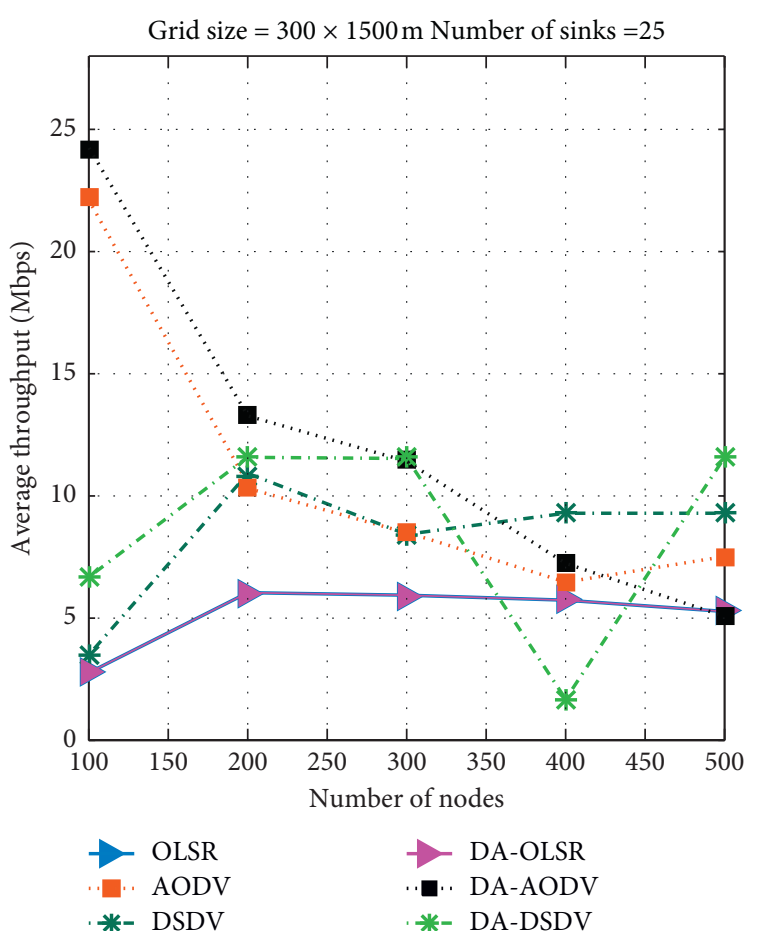

(a)

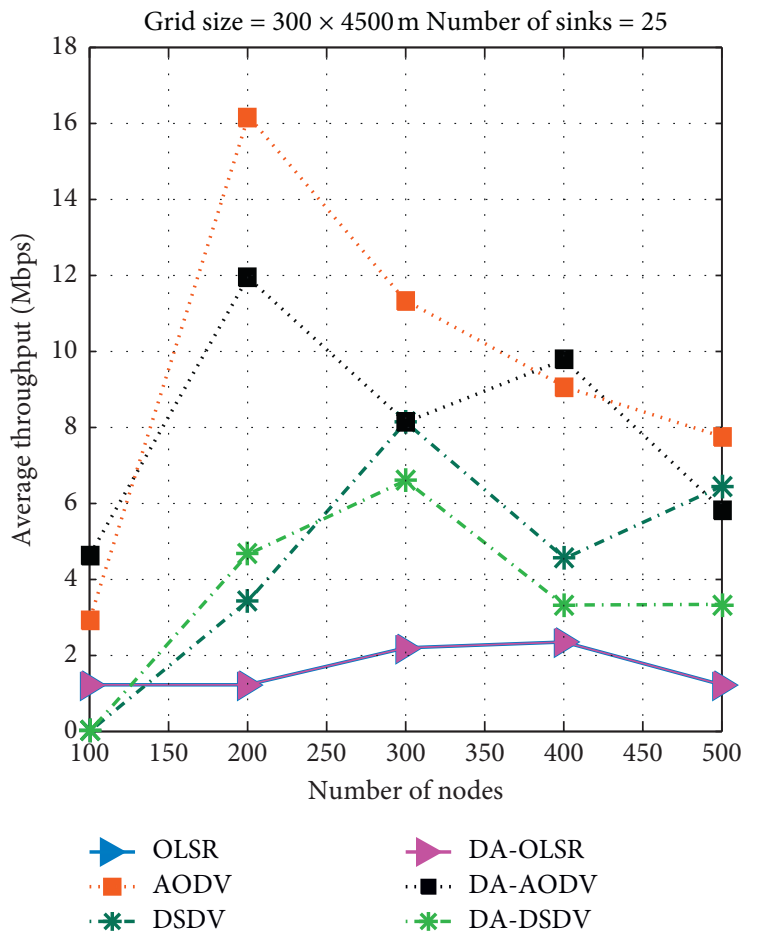

(c)

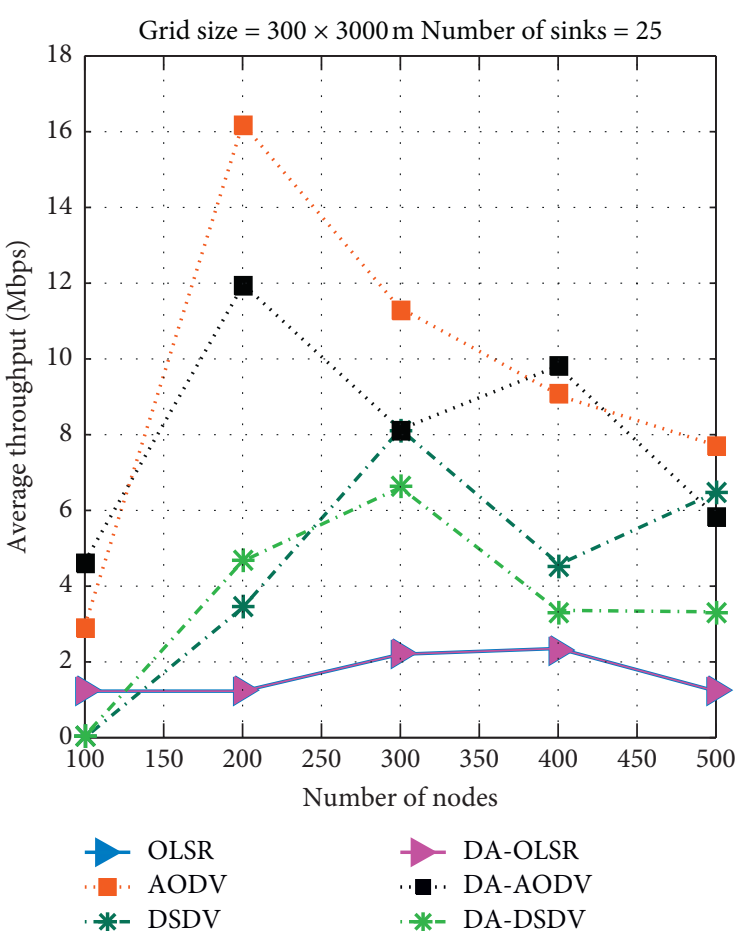

(b)

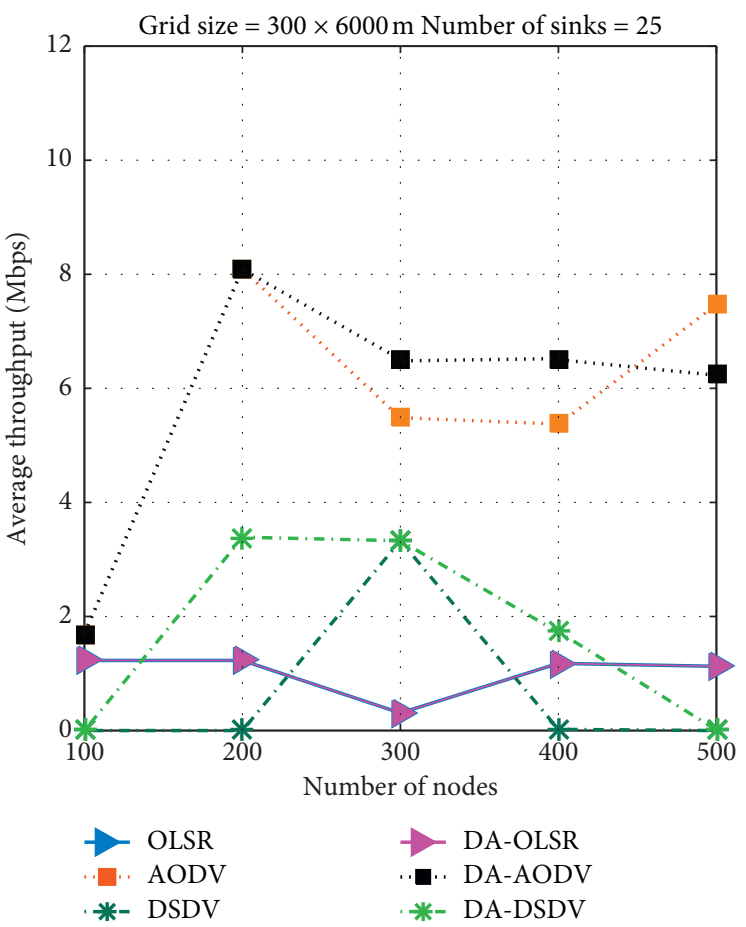

(d)

FIgURE 17: Comparison of average throughput of scenarios a and b with 25 sinks.

the protocols lie between DSDV and DA-AODV. When we compare the performance of traditional VANET routing protocols to that of the drone-assisted ones, it becomes clear that drone-assisted protocols show less average throughput for smaller grids, but these protocols have a comparatively enhanced performance for larger grids. The results presented in Figures 18(a)-18(d) are for the same number of sink nodes, i.e., 50, and vehicular nodes for the presented four graphs are from 100 to 500 . The grid size is initially $300 \times 1500 \mathrm{~m}$ and reaches up to $300 \times 6000 \mathrm{~m}$ with a constant interval of $1500 \mathrm{~m}$ along the $y$-axis. One more thing to be noted is that the greater the number of sink nodes is, the 


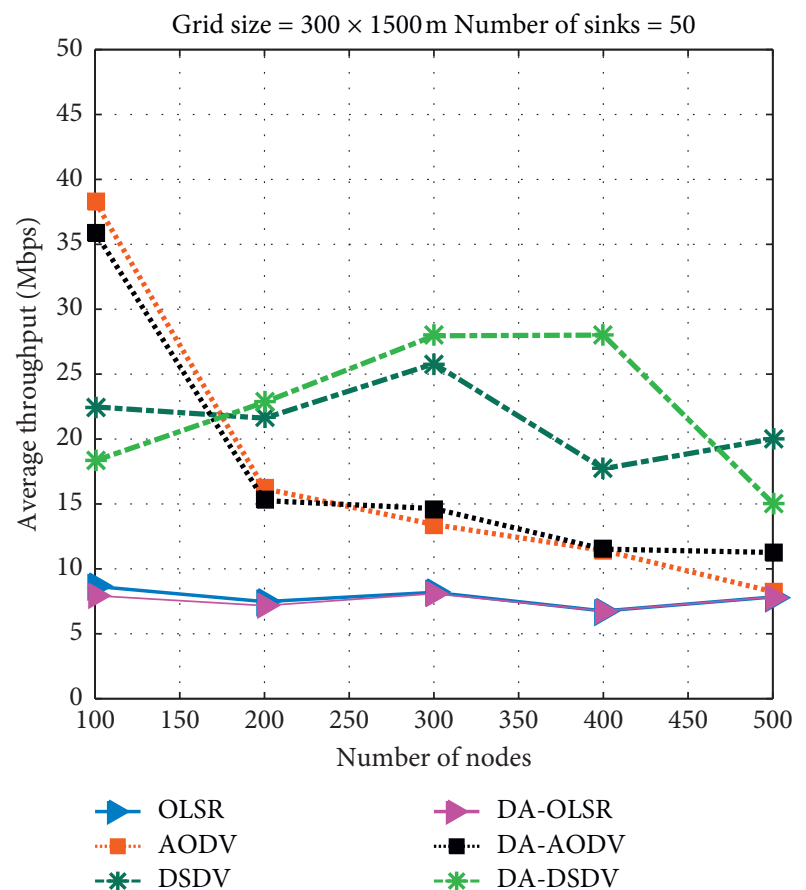

(a)

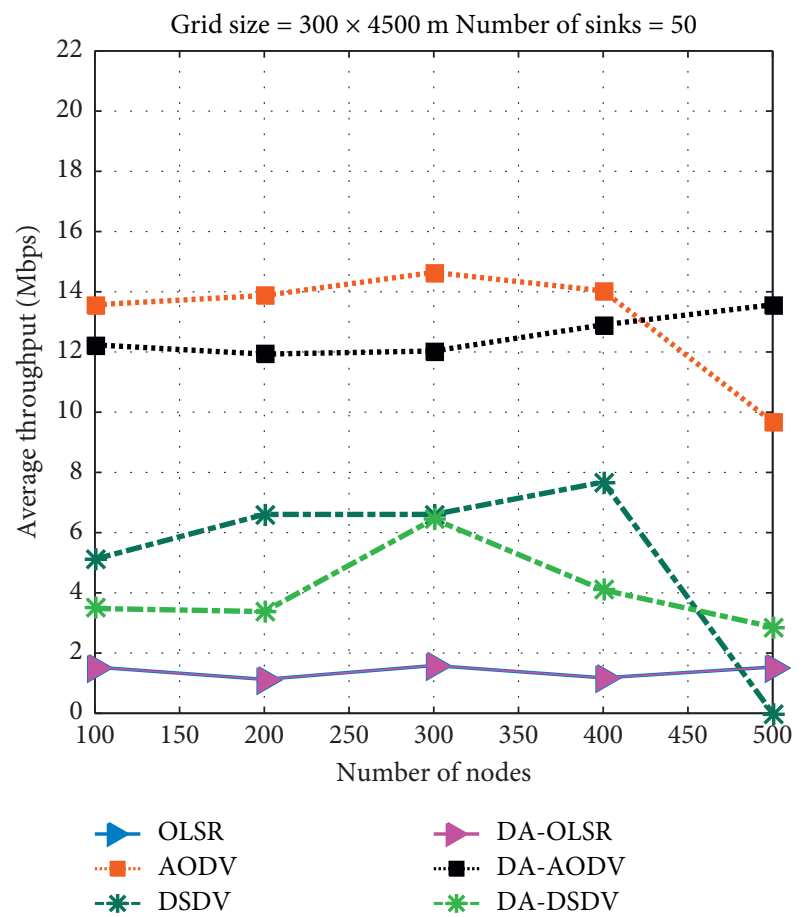

(c)

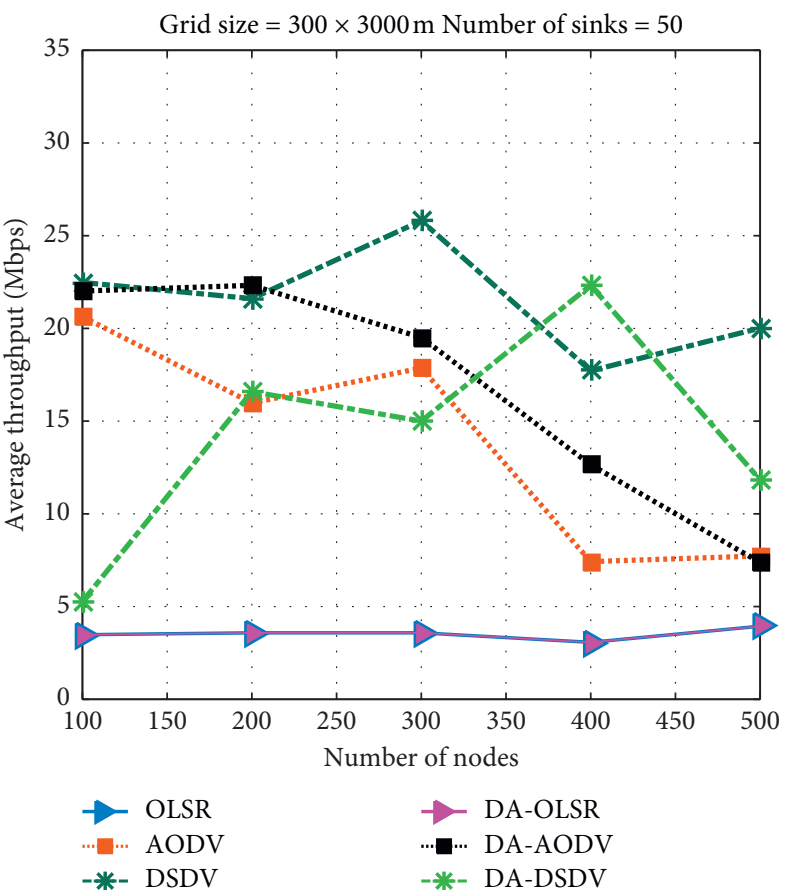

(b)

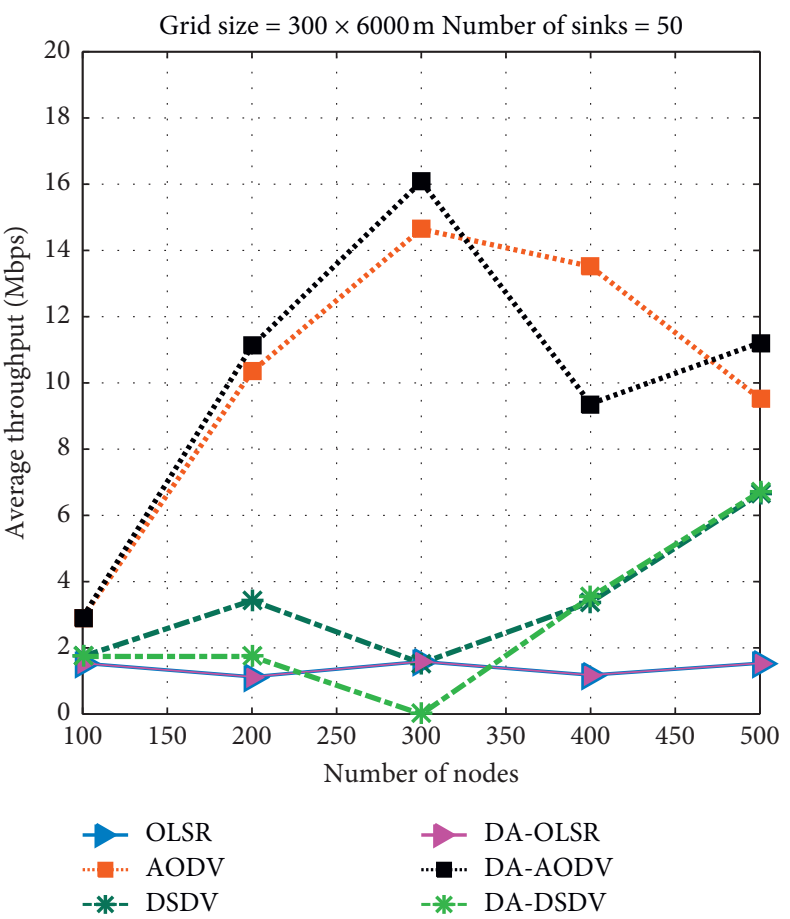

(d)

FIgURE 18: Comparison of average throughput of scenarios a and b with 50 sinks.

higher the performance of protocols will be. As can be seen from Figures 17 and 18, all the protocols have better performance when we have the number of sinks $=50$, especially the drone-assisted protocols.
6.5. Average Packet Delivery Ratio with 25 Sink Nodes. Figures 19(a)-19(d) present the comparative analysis of average packet delivery ratio in traditional VANET protocol and our proposed strategy at grid size of $300 \times 1500 \mathrm{~m}$ to 


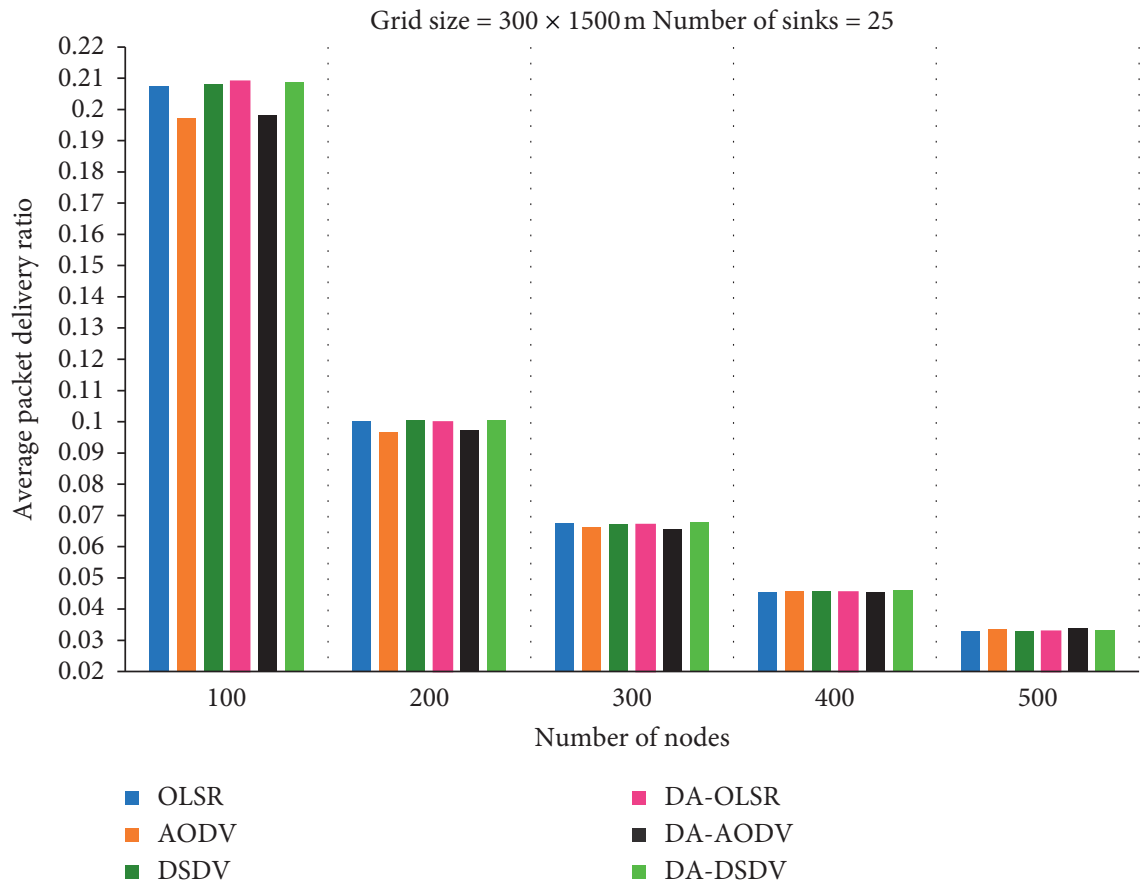

(a)

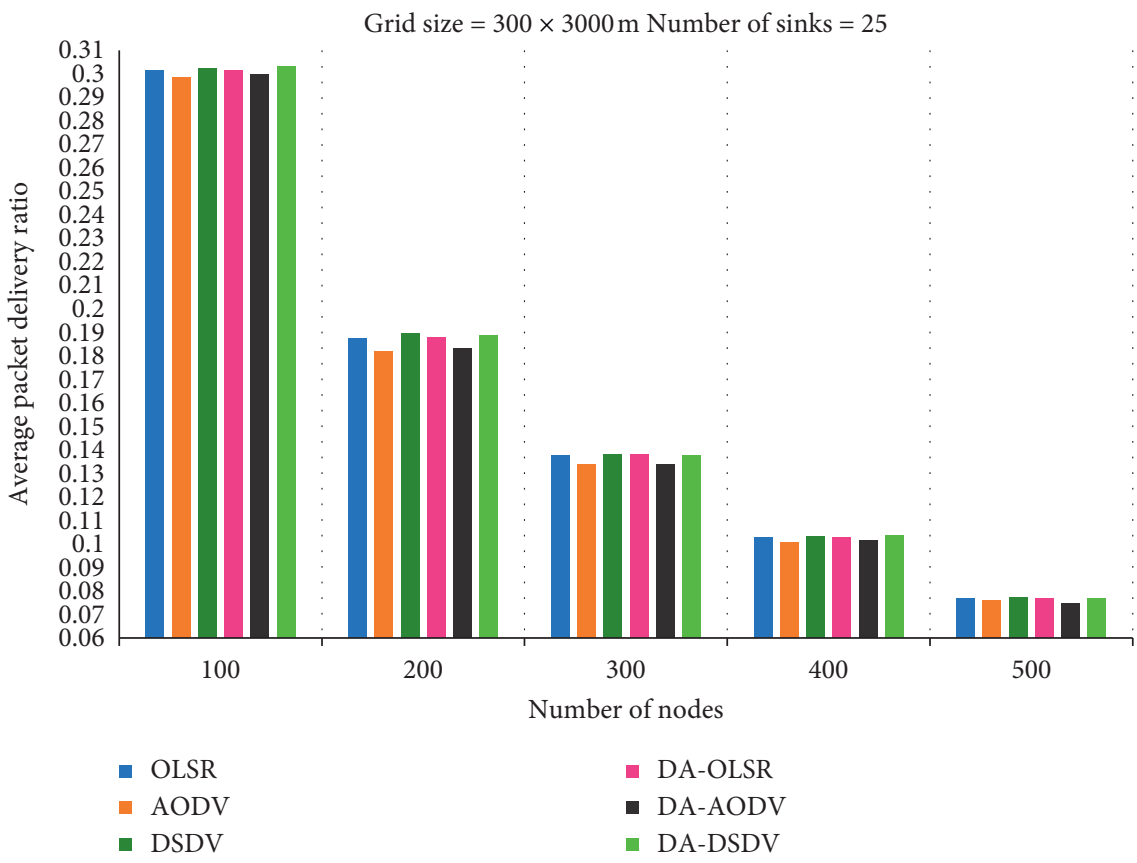

(b)

Figure 19: Continued. 


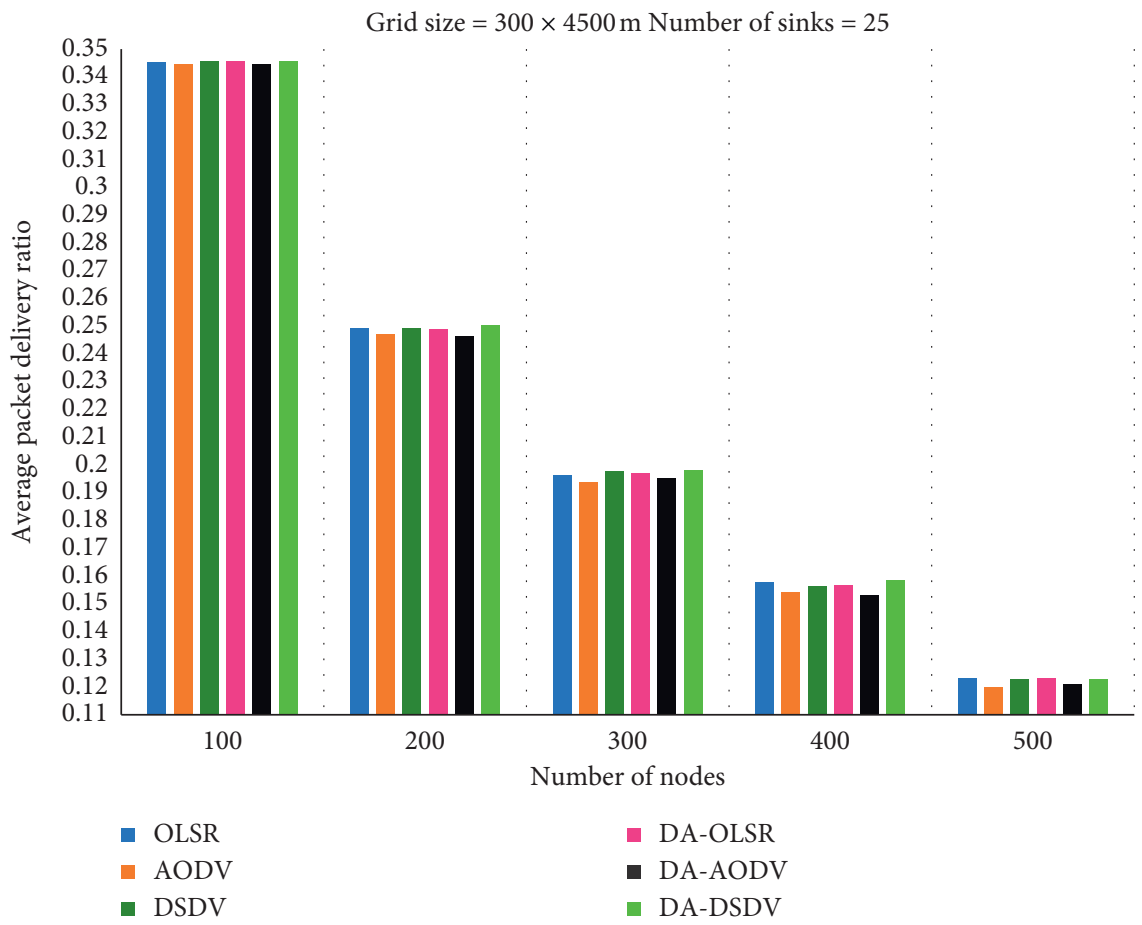

(c)

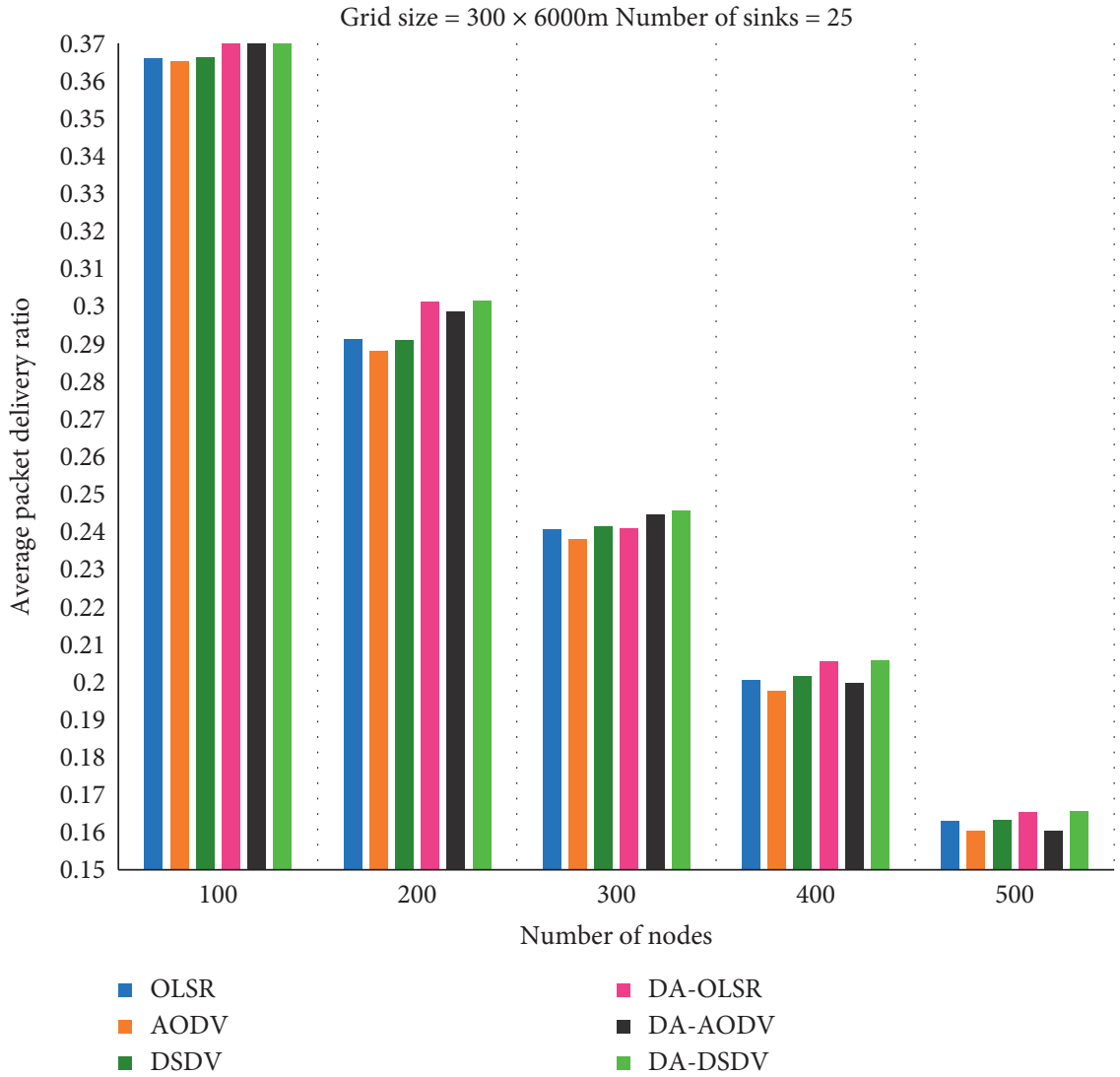

(d)

Figure 19: Comparison of Average PDR of scenarios a and b with 25 sinks. 


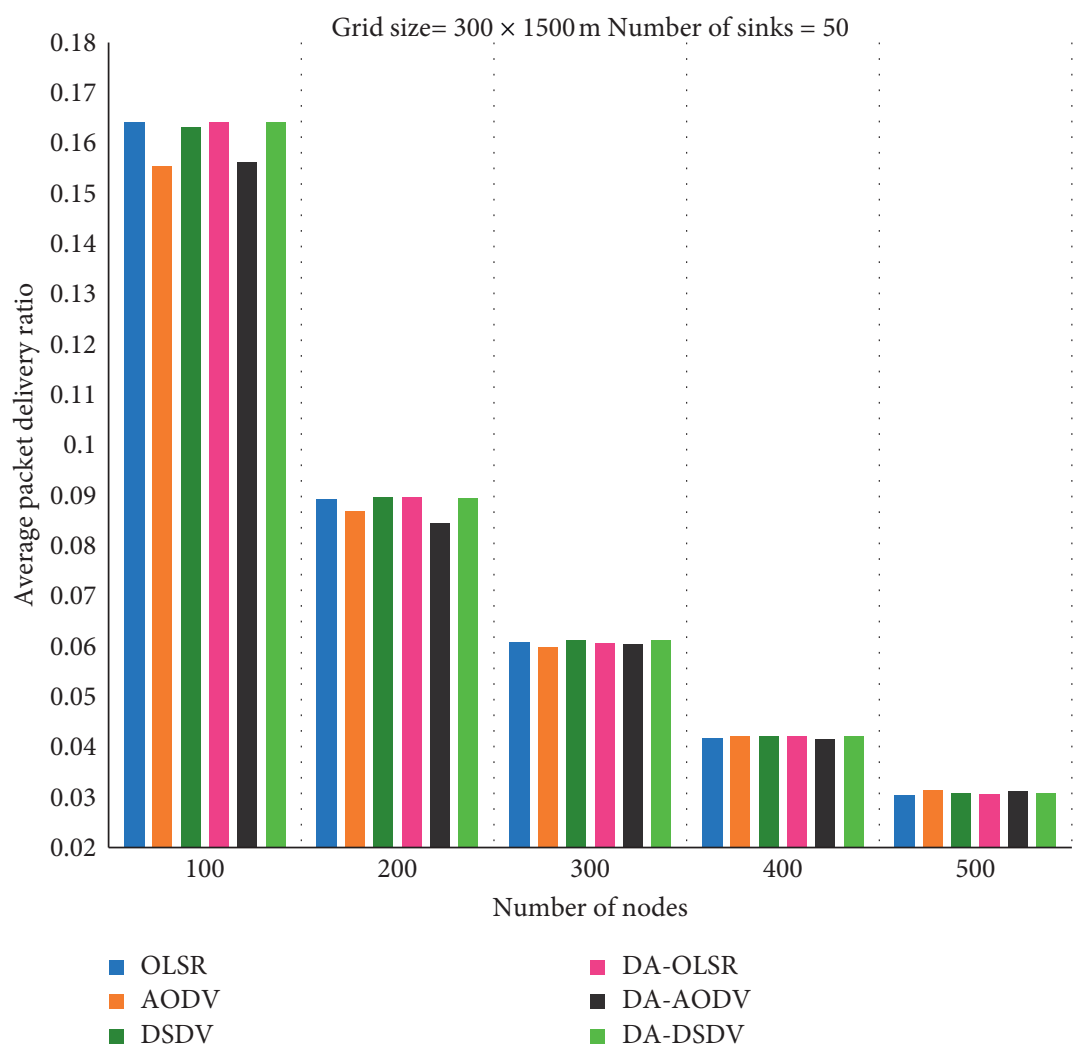

(a)

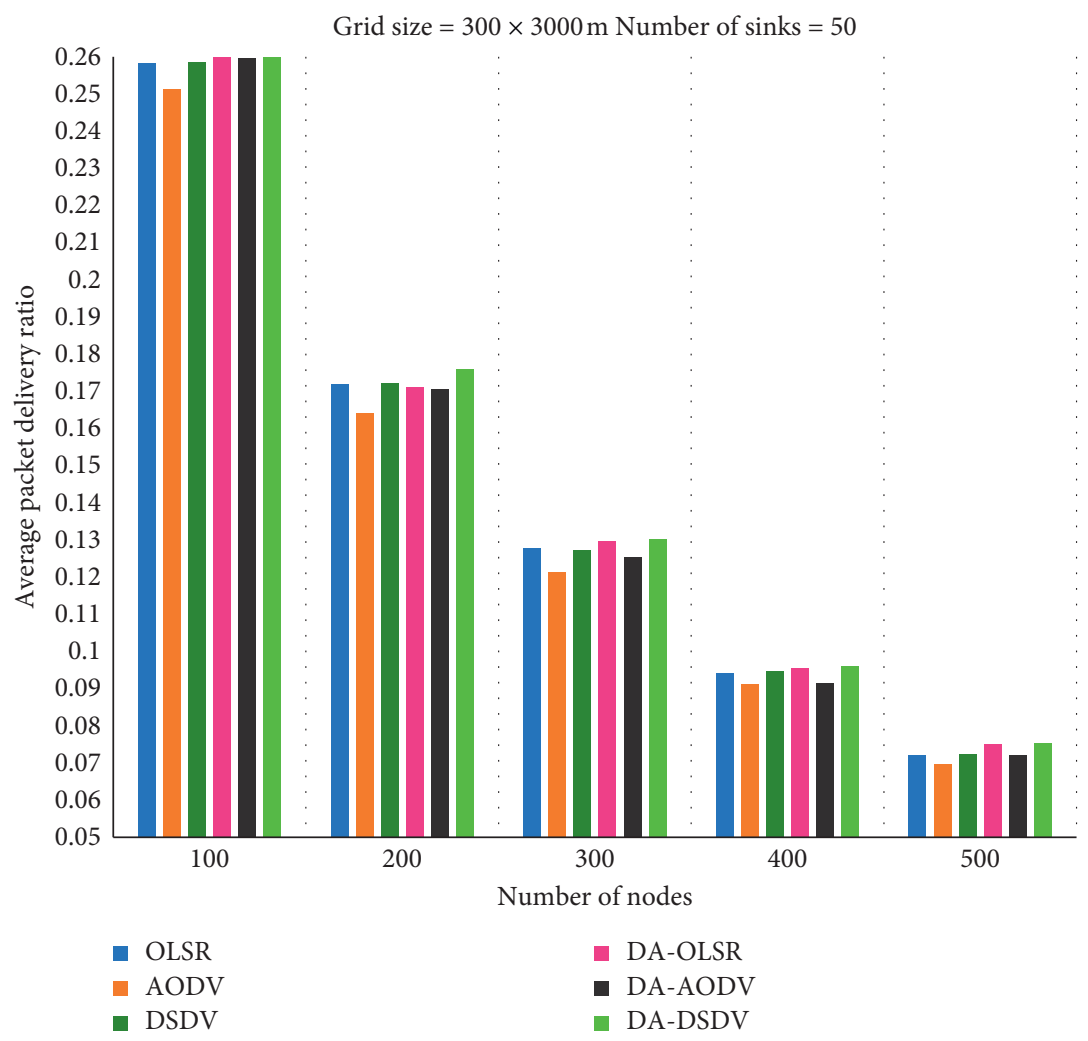

(b)

Figure 20: Continued. 


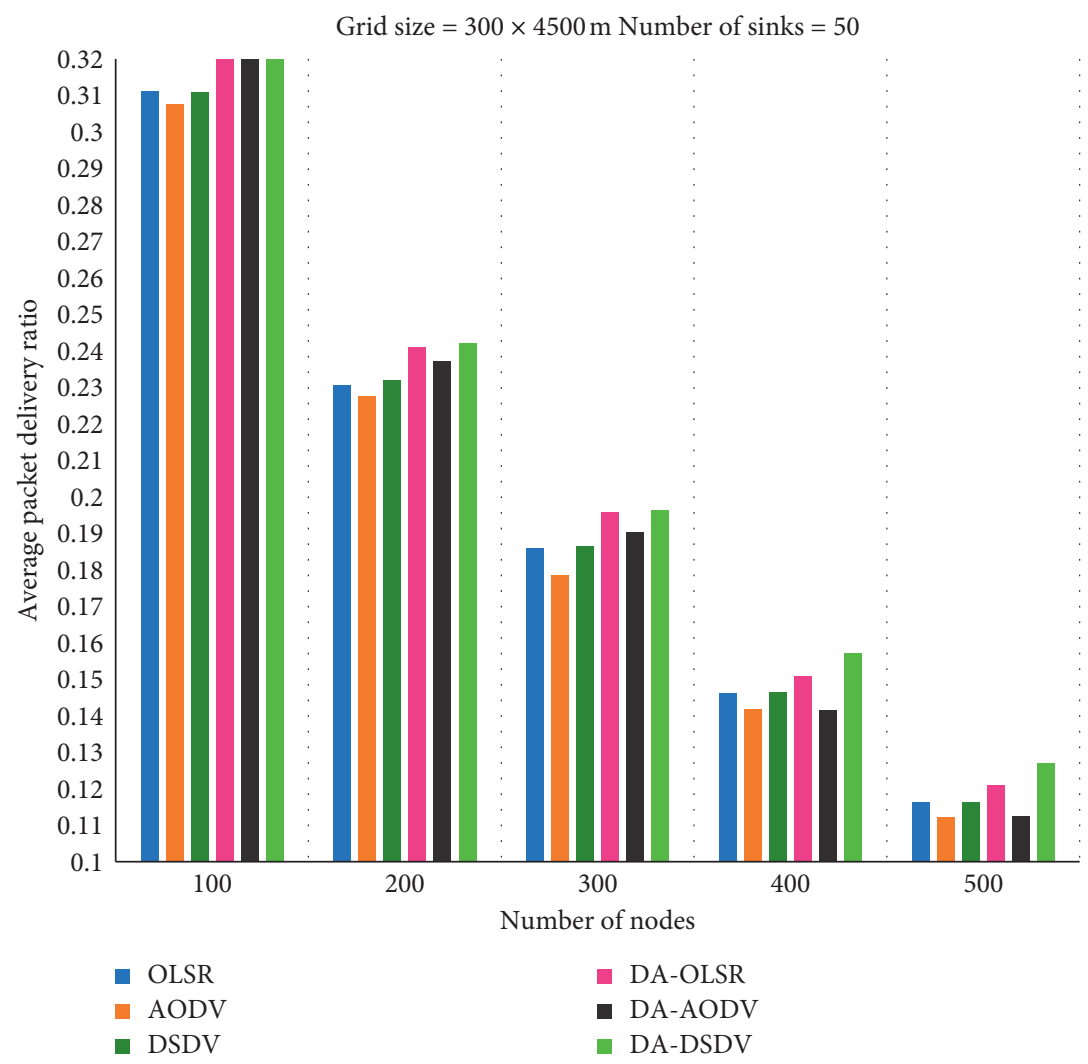

(c)

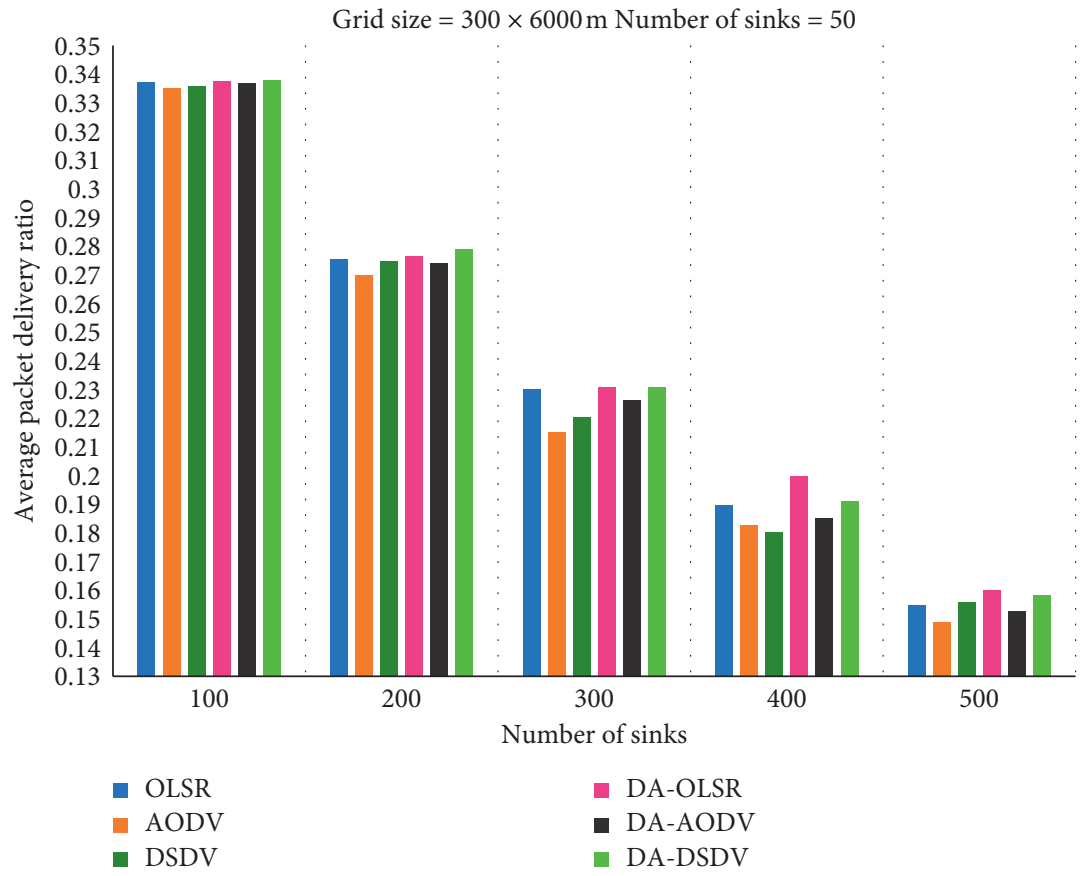

(d)

Figure 20: Comparison of average PDR of scenarios a and b with 50 sinks.

$300 \times 6000 \mathrm{~m}$ with 25 sink nodes in each case. In smaller grid size, the performance of the traditional routing protocol and that of our drone-assisted protocol are very close to each other, but as we move further towards greater grid size, the performance of our proposed drone-assisted protocols begins to enhance. As can be seen from Figures 19(a)-19(d), the performance of DA-OLSR and DA-DSDV is better than that of traditional OLSR and DSDV. Though AODV and 
DA-AODV could not perform significantly, the overall performance of our proposed strategy is better at a bigger grid size.

6.6. Average Packet Delivery Ratio with 50 Sink Nodes. Figures 20(a)-20(d) present the comparative analysis of average packet delivery ratio in traditional VANET protocol and our proposed strategy at grid size of $300 \times 1500 \mathrm{~m}$ to $300 \times 6000 \mathrm{~m}$ with 50 sink nodes in each case. In smaller grid size, the performance of the traditional routing protocol and that of our drone-assisted protocol are very close to each other, but as we move further towards greater grid size, the performance of our proposed drone-assisted protocols begins to enhance. As can be seen from Figures 20(a)-20(d), the performance of DA-OLSR, DA-AODV, and DA-DSDV is better than that of traditional OLSR, AODV, and DSDV. Therefore, we can conclude that the performance of our desired strategy is even better with 50 sinks as compared to traditional VANET.

\section{Conclusion and Future Work}

IoV is the new form of VANET and is the alliance of Internet and IoT. Internet of Vehicles is emerging as an important class of networks in the modern era, because of the immense traffic on the road, congested vehicular environment, and increased chance of vehicular collision. Many strategies have been proposed. The main concern of the researchers is improving the overall efficiency of IoT. The efficiency parameters may be greater average throughput, enhanced packet delivery ratio, less MAC/PHY overhead focus, less end-to-end delay, and minimum packet drop ratio. Our focus in this research is on providing such an efficient routing protocol that can help us in providing greater average throughput, enhanced packet delivery ratio, and less MAC/PHY overhead. For this purpose, we have made use of aerial nodes. We did so by elevating the sink nodes to a height greater than that we have in traditional routing protocols.

Extensive simulations have been carried out for traditional VANET routing protocols and drone-assisted routing protocol. The results have been generated and presented in graphical form. The output results have been analyzed one by one. Later, these results have been compared for both the traditional VANET and the one deployed using aerial nodes. This comparison helped us to understand that the assistance of aerial nodes helped us to enhance network efficiency. We have changed the grid size from $300 \times 1500 \mathrm{~m}$ to $300 \times 6000 \mathrm{~m}$ with an interval of $1500 \mathrm{~m}$ each time. We have also experimented with different numbers of sink nodes, i.e., 25 and 50. From all the experimentation and results gathered, we conclude that our proposed strategy performs well in terms of average throughput and average packet drop ratio when we have a bigger grid. Moreover, the number of sinks also affects these parameters; that is, the greater the number of aerial nodes is, the greater the performance of these parameters will be.

In the case of MAC/PHY overhead, although it increases with the increase in the number of vehicular nodes, its values are less with a greater number of aerial nodes than those of traditional routing protocols. In the future, we will implement our strategy by varying the transmission ranges. The grid sizes may also be increased along the $x$-axis as well as along the $y$-axis. Such proposed scheme will not only help in having better network experience in traffic, but also enhance the medicine and healthcare, agriculture, disaster, and emergency scenarios and provide environmental and surrounding information and a better solution for communication over a congested road. The topological constraint changes made produce novelty in our suggested scheme. We intend to find the average packet drop ratio and the end-toend delay in the future and to analyze the performance of our proposed strategy.

\section{Data Availability}

The data used to support the findings of this study are included in the article.

\section{Conflicts of Interest}

The authors declare that they have no conflicts of interest.

\section{References}

[1] M. Houmer and M. L. Hasnaoui, "A qualitative assessment of VANET routing protocols under different mobility models," Journal of Computer Science, vol. 15, no. 1, pp. 161-170, 2019.

[2] J. Wang, X. Xiao, and P. Lu, "A survey of vehicular ad hoc network routing protocols," Journal of Electrical and Electronic Engineering, vol. 7, no. 2, pp. 46-50, 2019.

[3] J. P. West and J. S. Bowman, "The domestic use of drones: an ethical analysis of surveillance issues," Public Administration Review, vol. 76, no. 4, pp. 649-659, 2016.

[4] S. K. Lakshmanaprabu, K. Shankar, S. Sheeba Rani et al., "An effect of big data technology with ant colony optimization based routing in vehicular ad hoc networks: towards smart cities," Journal of Cleaner Production, vol. 217, pp. 584-593, 2019.

[5] N. Melaouene and R. Romadi, "An enhanced routing algorithm using ant colony optimization and VANET infrastructure," MATEC Web of Conferences, vol. 259, Article ID 02009, 2019.

[6] S. R. Yahiabadi, B. Barekatain, and K. Raahemifar, "TIHOO: an enhanced hybrid routing protocol in vehicular ad-hoc networks," EURASIP Journal on Wireless Communications and Networking, vol. 2019, no. 1, p. 192, 2019.

[7] N. K. Chaubey, "Security analysis of vehicular ad hoc networks (VANETs): a comprehensive study," International Journal of Security and Its Applications, vol. 10, no. 5, pp. 261-274, 2016.

[8] A. Ahamed and H. Vakilzadian, "Issues and challenges in VANET routing protocols," in Proceedings of the 2018 IEEE International Conference on Electro/Information Technology (EIT), pp. 0723-0728, IEEE, Rochester, MI, USA, May 2018.

[9] A. Awang, K. Husain, N. Kamel, and S. Aissa, "Routing in vehicular ad-hoc networks: a survey on single- and cross-layer design techniques, and perspectives," IEEE Access, vol. 5, pp. 9497-9517, 2017.

[10] N. Chowdhary and P. D. Kaur, "Addressing the characteristics of mobility models in IoV for smart city," in Proceedings of the 2016 International Conference on Computing, Communication 
and Automation (ICCCA), pp. 1298-1303, IEEE, Greater Noida, India, April 2016.

[11] R. C. Manurung, D. Perdana, and R. Munadi, "Performance evaluation Gauss-Markov mobility model in vehicular ad-hoc network with spearman correlation coefficient," in Proceedings of the 2016 International Seminar on Intelligent Technology and Its Applications (ISITIA), pp. 351-356, IEEE, Lombok, Indonesia, July 2016.

[12] P. E. Ramadhani, M. D. Setiawan, M. A. Yutama, D. Perdana, and R. F. Sari, "Performance evaluation of hybrid wireless mesh protocol (HWMP) on VANET using VanetMobiSim," in Proceedings of the 2016 International Conference on Computational Intelligence and Cybernetics, pp. 41-46, IEEE, Makassar, Indonesia, November 2016.

[13] B. Ramakrishnan, M. Selvi, and R. B. Nishanth, "Efficiency measure of routing protocols in vehicular ad hoc network using freeway mobility model," Wireless Networks, vol. 23, no. 2, pp. 323-333, 2017.

[14] M. Arif, G. Wang, M. Zakirul Alam Bhuiyan, T. Wang, and J. Chen, "A survey on security attacks in VANETs: communication, applications and challenges," Vehicular Communications, vol. 19, Article ID 100179, 2019.

[15] C. Jayapal and S. S. Roy, "Road traffic congestion management using VANET," in Proceedings of the 2016 International Conference on Advances in Human Machine Interaction (HMI), pp. 1-7, IEEE, Bangalore, India, March 2016.

[16] S. Singh, S. Negi, S. K. Verma, and N. Panwar, "Comparative study of existing data scheduling approaches and role of cloud in VANET environment," Procedia Computer Science, vol. 125, pp. 925-934, 2018.

[17] C. Chen, L. Liu, T. Qiu, K. Yang, F. Gong, and H. Song, "ASGR: an artificial spider-web-based geographic routing in heterogeneous vehicular networks," IEEE Transactions on Intelligent Transportation Systems, vol. 20, no. 5, pp. 16041620, 2018.

[18] R. A. Nazib and S. Moh, "Routing protocols for unmanned aerial Vehicle-Aided vehicular Ad Hoc networks: a survey," IEEE Access, vol. 8, pp. 77535-77560, 2020.

[19] O. Sami Oubbati, N. Chaib, A. Lakas, S. Bitam, and P. Lorenz, "U2RV: UAV-assisted reactive routing protocol for VANETs," International Journal of Communication Systems, vol. 33, no. 10, Article ID e4104, 2020.

[20] N. Lin, L. Fu, L. Zhao, G. Min, A. Al-Dubai, and H. Gacanin, "A novel multimodal collaborative drone-assisted VANET networking model," IEEE Transactions on Wireless Communications, vol. 19, no. 7, pp. 4919-4933, 2020.

[21] A. Kumar, K. Rajalakshmi, S. Jain, A. Nayyar, and M. Abouhawwash, "A novel heuristic simulation-optimization method for critical infrastructure in smart transportation systems," International Journal of Communication Systems, vol. 33, no. 11, Article ID e4397, 2020.

[22] T. Lu, S. Chang, and W. Li, "Fog computing enabling geographic routing for urban area vehicular network," Peer-toPeer Networking and Applications, vol. 11, no. 4, pp. 749-755, 2018.

[23] M. Bhatt, S. Sharma, A. K. Luhach, and A. Prakash, "Nature inspired route optimization in vehicular adhoc network," in Proceedings of the 2016 5th International Conference on Reliability, Infocom Technologies and Optimization (Trends and Future Directions)(ICRITO), pp. 447-451, IEEE, Noida, India, September 2016.

[24] M. Dorigo, M. Birattari, and T. Stutzle, "Ant colony optimization," IEEE Computational Intelligence Magazine, vol. 1, no. 4, pp. 28-39, 2006.
[25] M. Farhanchi, R. Hassanzadeh, I. Mahdavi, and N. MahdaviAmiri, "A modified ant colony system for finding the expected shortest path in networks with variable arc lengths and probabilistic nodes," Applied Soft Computing, vol. 21, pp. 491-500, 2014.

[26] K. Prakash, P. C. Philip, R. Paulus, and A. Kumar, "A packet fluctuation-based OLSR and efficient parameters-based OLSR routing protocols for urban vehicular ad hoc networks," in Recent Trends in Communication and Intelligent Systems, pp. 79-87, Springer, Berlin, Germany, 2020.

[27] X. Bao, H. Li, G. Zhao, L. Chang, J. Zhou, and Y. Li, "Efficient clustering V2V routing based on PSO in VANETs," Measurement, vol. 152, Article ID 107306, 2020.

[28] M. T. Abbas, A. Muhammad, and W.-C. Song, "Road-aware estimation model for path duration in Internet of vehicles (IoV)," Wireless Personal Communications, vol. 109, no. 2, pp. 715-738, 2019.

[29] V. Jindal and P. Bedi, "An improved hybrid ant particle optimization (IHAPO) algorithm for reducing travel time in VANETs," Applied Soft Computing, vol. 64, pp. 526-535, 2018.

[30] D. Zhang, T. Zhang, and X. Liu, "Novel self-adaptive routing service algorithm for application in VANET," Applied Intelligence, vol. 49, no. 5, pp. 1866-1879, 2019.

[31] D. Tian, K. Zheng, J. Zhou, Z. Sheng, Q. Ni, and Y. Wang, "Unicast routing protocol based on attractor selection model for vehicular ad-hoc networks," in Proceedings of the International Conference on Internet of Vehicles, pp. 138-148, Springer, Nadi, Fiji, December 2016.

[32] M. Elhoseny and K. Shankar, "Energy efficient optimal routing for communication in VANETs via clustering model," in Emerging Technologies for Connected Internet of Vehicles and Intelligent Transportation System Networks, pp. 1-14, Springer, Berlin, Germany, 2020.

[33] A. Nayyar, "Flying adhoc network (FANETs): simulation based performance comparison of routing protocols: AODV, DSDV, DSR, OLSR, AOMDV and HWMP," in Proceedings of the 2018 International Conference on Advances in Big Data, Computing and Data Communication Systems (icABCD), pp. 1-9, IEEE, Durban, South Africa, August 2018.

[34] A. V. Leonov, "Application of bee colony algorithm for FANET routing," in Proceedings of the 2016 17th International Conference of Young Specialists on Micro/Nanotechnologies and Electron Devices (EDM), pp. 124-132, IEEE, Erlagol, Altai, June 2016.

[35] S. Majumdar, P. R. Prasad, S. S. Kumar, and K. S. Kumar, "An efficient routing algorithm based on ant colony optimisation for VANETs," in Proceedings of the 2016 IEEE International Conference on Recent Trends in Electronics, Information \& Communication Technology (RTEICT), pp. 436-440, IEEE, Bangalore, India, May 2016.

[36] O. S. Oubbati, A. Lakas, F. Zhou, M. Güneş, and M. B. Yagoubi, "A survey on position-based routing protocols for flying ad hoc networks (FANETs)," Vehicular Communications, vol. 10, pp. 29-56, 2017.

[37] V. Saritha, P. V. Krishna, S. Misra, and M. S. Obaidat, "Learning automata based optimized multipath routingusing leapfrog algorithm for VANETs," in Proceedings of the 2017 IEEE International Conference on Communications (ICC), pp. 1-5, IEEE, Paris, France, January 2017.

[38] J. F. Bravo-Torres, M. López-Nores, Y. Blanco-Fernández, J. J. Pazos-Arias, M. Ramos-Cabrer, and A. Gil-Solla, "Optimizing reactive routing over virtual nodes in VANETs," IEEE Transactions on Vehicular Technology, vol. 65, no. 4, pp. 2274-2294, 2015. 
[39] M. Dixit, R. Kumar, and A. K. Sagar, "VANET: architectures, research issues, routing protocols, and its applications," in Proceedings of the 2016 International Conference on Computing, Communication and Automation (ICCCA), pp. 555561, IEEE, Greater Noida, India, April 2016.

[40] V. A. Maistrenko, L. V. Alexey, and V. A. Danil, "Experimental estimate of using the ant colony optimization algorithm to solve the routing problem in FANET," in Proceedings of the 2016 International Siberian Conference on Control and Communications (SIBCON), pp. 1-10, IEEE, Moscow, Russia, May 2016.

[41] Y. Zeng, R. Zhang, and T. J. Lim, "Throughput maximization for UAV-enabled mobile relaying systems," IEEE Transactions on Communications, vol. 64, no. 12, pp. 4983-4996, 2016.

[42] M. Mozaffari, W. Saad, M. Bennis, and M. Debbah, "Mobile unmanned aerial vehicles (UAVs) for energy-efficient internet of things communications," IEEE Transactions on Wireless Communications, vol. 16, no. 11, pp. 7574-7589, 2017.

[43] A. Yasser, M. Zorkany, and N. Abdel Kader, "VANET routing protocol for $\mathrm{V} 2 \mathrm{~V}$ implementation: a suitable solution for developing countries," Cogent Engineering, vol. 4, no. 1, Article ID 1362802, 2017. 